\title{
12. PALEOENVIRONMENT AND BIOSTRATIGRAPHY OF FORAMINIFERS AT SITES 794, 795, 796, AND 797 IN THE JAPAN SEA ${ }^{1}$
}

\author{
Charlotte A. Brunner ${ }^{2}$
}

\begin{abstract}
Biostratigraphy and paleoenvironmental history of deep and surficial waters of the Japan Sea are addressed using sequences recovered from the floor of the backarc basin. The study is divided into two parts: (1) foraminifer biostratigraphy and paleoenvironmental assessment of sedimentary sequences recovered from above igneous basement at the four sites and (2) detailed planktonic foraminifer paleoenvironmental analysis of Quatemary and Pliocene sequences from Sites 794 and 797 in the Yamato Basin.

A total of 253 samples were examined for the foraminifer biostratigraphy and 325 samples for the detailed paleoenvironmental study of Quaternary and Pliocene sequences. Low abundance and sporadic occurrence of foraminifers limited interpretation of results. Foraminifer-bearing intervals were correlated where possible to diatom and calcareous nannofossil zonations, and the sequences were successfully assigned to the foraminifer zonation of Matsunaga. Unfortunately, extensive barren intervals and sporadic occurrences of planktonic foraminifers prevented zonation of Quaternary and Pliocene intervals, although some interesting conclusions about paleoenvironment were possible and are listed below.

A sequence of Neogene (sensu lato) paleoenvironmental events were identified: (1) deepening of the Yamato basins to middle bathyal depths by the early to middle Miocene, an event contemporaneous with the age of some deep basins known from uplifted sections adjacent to the Japan Basin; (2) cooling of the Japan Sea in the early middle Miocene; (3) oxygenation of deep waters in the late Miocene; (4) further cooling of surficial water masses between the Olduvai Subchron and the Brunhes/Matuyama Boundary; and (5) extermination of lower middle bathyal faunas and replacement by upper middle bathyal faunas near the base of the Quaternary.
\end{abstract}

\section{INTRODUCTION}

A fundamental objective long sought in the Japan Sea region is a continuous biostratigraphic and paleoenvironmental record of the marginal sea. The scientific reward from such a sequence includes issues of multidisciplinary interest. The paleoenvironmental record of the backarc basin could detail the events surrounding the opening and subsidence of the nascent basin, its maturation, and the effects of compression that may lead ultimately to its destruction. Rotation of the island arc during Miocene basin widening along with formation of sills across passages to the open Pacific Ocean probably are recorded by assemblage changes in the biota of both the upper water column and in bottom waters. The late Pliocene and Quaternary facies (light and dark lithologic cycles) suggest that the basin may have acted like an amplifier in response to glacial climatic cycles. The development of a continuous biostratigraphy correlated to the global time scale could place these events in historical perspective relative to those in the Pacific Ocean and elsewhere.

The taxonomy of Japan foraminifers, the foraminifer biostratigraphy, and paleoenvironments of uplifted marine sections on western Honshu and Hokkaido have been discussed by many workers (i.e., Asano, 1950-1951; Saito, 1963; Matsunaga, 1963; Asano, et al., 1969; Burckle and Todd, 1976; Kurihara, 1976; Tsuchi et al., 1984; Oda, 1986). Several major events in the history of the Japan Sea were identified from these sequences.

1. Uplifted bathyal basins are as old as the late early Miocene, a date that marks a youngest age for bathyal depth basins during the birth process of the backarc basin (i.e., Matoba, 1984).

2. The nascent Japan Sea supported a warm subtropical planktonic fauna in the early Miocene, but was cool by the late middle Miocene (i.e., Asano et al., 1969; Saito, 1963; Matoba, 1984; Tsuchi et al., 1984). This cooling event may be related to clockwise rotation of the

\footnotetext{
'Pisciotto, K. A., Ingle, J. C., Jr., von Breymann, M. T., Barron, J., et al., 1992. Proc. ODP, Sci. Results, 127/128, Pt. 1: College Station, TX (Ocean Drilling Program).

${ }^{2}$ Center for Marine Science, University of Southern Mississippi, Stennis Space Center, MS 39529.
}

island arc, a process that closed southerly passages and opened northerly passages to the Pacific Ocean (Chinzei, 1986).

3 . The sedimentary sequence between the early Miocene and Pliocene contains sporadic occurrences of foraminifers, making it very difficult to piece together a history during basin growth and maturity (i.e., Matsunaga, 1963).

4. Middle Miocene to late Pliocene agglutinated foraminifer faunas of the Onnagawa and Funakawa Formations indicate low oxygen conditions in the deep waters of the bathyal basins (i.e., Matoba, 1984). The overlying Pleistocene fauna, which is dominated by calcareous benthic foraminifers, marks a change in deep water to more oxygenated conditions.

5. Matoba (1984, and references therein) has noted that the late Pliocene to early Pleistocene fauna typified in the Kitaura and Wakimoto Formations is quite different from the latest Quaternary fauna recovered in piston cores, and he suggests that a drastic change in bathyal oceanographic conditions triggered a major faunal turnover in the late Pleistocene.

The objective of this paper is to assess the capability of the data set to address the goals listed above, to expand on the history known from uplifted sections, and to identify additional significant events since the birth of the marginal sea.

\section{METHODS}

\section{Neogene Biostratigraphy and Paleoenvironmental Assessment}

Core-catcher samples were prepared from sediment cores of the four sites, and in a few instances samples were taken from within cores. 253 samples approximately $20 \mathrm{~cm}^{3}$ in volume were dried, soaked in a hot $1 \%$ Calgon solution, washed on a sieve of $63-\mu \mathrm{m}$ openings, and inspected for foraminifers. In most samples, all specimens were picked and identified to genus or species. In samples with common to abundant foraminifers, representatives of each species present were picked (see Tables 2 and 3 in the Biostratigraphy section of each site chapter in Tamaki, Pisciotto, Allan, et al., 1990). The species ranges and assemblages were compared with the zonation of Matsunaga (1963). 


\section{Detailed Quaternary and Pliocene Biostratigraphy and Paleoenvironmental Analysis}

Samples were taken from each section of the hydraulically pistoncored portions at Sites 794 and 797. As many as five samples per section were taken in the top five and six cores respectively, and one sample per section from deeper in the sequences. Samples were weighed, dried, and soaked in a hot $1 \%$ Calgon solution for about $3 \mathrm{hr}$. The disaggregated sediment was washed on a sieve of $63-\mu \mathrm{m}$ openings, and the sand-size residue was dried, weighed, and inspected for foraminifers. Samples with abundant planktonic foraminifers were split into about 300 specimens using an Otto microsplitter. Planktonic foraminifers were identified to species and counted, benthic foraminifers were counted.

Several parameters were calculated from the measured data: sand percent by weight, planktonic and benthic foraminifer numbers (number of specimens per gram of sediment), benthic/planktonic ratio (number of benthic specimens/total number of foraminifer specimens), the coiling ratio of Neogloboquadrina pachyderma (number of sinistral forms/total number of Neogloboquadrina pachyderma tests), and relative frequency of 11 planktonic foraminifer species and counting groups. Q-mode cluster analysis was used to objectively separate planktonic foraminifer assemblages. The procedure used a simple Euclidean distance coefficient applied to the relative species frequency data and amalgamated samples using the smallest average distance between groups. The results were compared to the biostratigraphy of Maiya et al. (1976) and Lagoe and Thompson (1988).

\section{RESULTS OF NEOGENE FORAMINIFER BIOSTRATIGRAPHY}

Foraminifers are generally rare (Table 1) and samples with common to abundant foraminifers are, with a few notable exceptions, limited to the Quaternary and upper Pliocene. Of 65 samples examined at Site 794 (Tables 1 and 2), 27 are barren, 20 have fewer than 10 specimens, and only 4 from the Quaternary sequence have more than 100 specimens, which are dominated by calcareous planktonic foraminifers. Of 68 samples examined at Site 795 (Tables 1 and 3), 25 are barren, 7 have fewer than 10 specimens, and 20 have more than 100 specimens, which, in general, are dominated by agglutinated foraminifers. One sample from the middle Miocene sequence bears more than 100 calcareous foraminifers. Of 53 samples examined from Site 796 (Tables 1 and 4), 23 are barren, 11 have fewer than 10 specimens, and 6 have greater than 100 specimens. One of the latter samples is upper Miocene, and the others are Quaternary and upper Pliocene. Of 67 samples from Site 797 (Tables 1 and 5), 23 are barren, 12 have fewer than 10 specimens, and 13 have more than 100 specimens. Three of these lie in the Miocene, whereas the other 10 are from the Quaternary and upper Pliocene.

\section{Quaternary Foraminifers}

The Quaternary sequences (Koizumi, this volume; L. Vigliotti and J. Wippern, pers. comm., 1990) at all sites bear several features in

Table 1. Frequency of samples bearing foraminifers.

\begin{tabular}{cccccc}
\hline & \multicolumn{2}{c}{$\begin{array}{c}\text { Frequency of Samples with Specified Numbers } \\
\text { of Foraminifer Tests }\end{array}$} & $\begin{array}{c}\text { Total } \\
\text { Number } \\
\text { Site }\end{array}$ & \begin{tabular}{c} 
Barren \\
\cline { 2 - 4 } \\
of Samples
\end{tabular} \\
\hline 794 & 27 & 20 & 14 & 4 & 65 \\
795 & 25 & 7 & 16 & 20 & 68 \\
796 & 23 & 11 & 13 & 6 & 53 \\
797 & 23 & 12 & 19 & 13 & 67 \\
\hline
\end{tabular}

common (Tables 2 through 5). They contain almost no agglutinated foraminifers, and have several intervals with abundant subarctic to arctic planktonic foraminifers. The sequences contain some miliolids like Pyrgo, Nummoloculina, Quinqueloculina, and other genera, and they have assemblages with frequent Globocassidulina subglobosa, Islandiella norcrossi, Stainforthia complanata, Brizalina pacifica, Epistominella pacifica, Fursenkoina seminuda, Bulimina striata var. mexicana, and Trifarina. There are too few foraminifers in this set of data to clearly distinguish benthic assemblages within the Quaternary.

\section{Pliocene Foraminifers}

The most distinctive feature of the Pliocene intervals is the consistent range of Miliammina echigoensis throughout the four sites (Tables 2 through 5). At all holes except Hole 796B, the top of the range of this taxon lies within the Neodenticula koizumii Diatom Zone (Koizumi, this volume). At all holes except Hole 796B, the bottom of the range lies in the Thalassiosira oestrupii Diatom Zone (Koizumi, this volume). The deepest occurrence at Site 794 (Table 2) is a single specimen, which lies in the older Neodenticula kamtschatica Diatom Zone (Koizumi, this volume). This occurrence could be contamination from upsection as the next occurrence of Miliammina echigoensis lies within the Thalassiosira oestrupii Diatom Zone (Koizumi, this volume).

Species that occur in the Pliocene (Koizumi, this volume) at all four sites (Tables 2 through 5) include the agglutinated species Martinottiella communis, Eggerella bradyi, and Eggerella sp. A, and the calcareous species Melonis pompilioides. Species that occur at Sites 795, 796, and 797 include Chilostomella oolina and Globobulimina auriculata. The two sites from the Japan Basin also share Quinqueloculina akneriana and Oolina globosa. The two sites from the Yamato Basin, however, are difficult to compare because Site 794 has the worst calcium carbonate preservation and lowest diversity of the four sites, whereas Site 797 has the best calcium carbonate preservation and highest diversity of the four sites.

\section{Upper Miocene Foraminifers}

In the Japan Basin, the sequence within the Neodenticula kamtschatica Diatom Zone (Koizumi, this volume) is nearly barren of foraminifers. In the Yamato Basin, the upper Miocene sediments of Sites 794 and 797 are also near barren except at the base of the zone where Sample 127-797-31X-CC, is enriched with well preserved Globigerina bulloides, Globobulimina auriculata, Sigmavirgulina sp., Cibicides lobatulus, Elphidium subincertum, and traces of other species. This sample may be displaced from shallower depth.

Correlation of foraminifers to the diatom zonation of Koizumi (this volume) is difficult below the uppermost Miocene because of diagenesis below the opal-A/opal-CT transition, which lies at various levels within the upper Miocene at all four sites. Diatom zones are recognized where diatoms are preserved in carbonate concretions. Additionally, calcareous nannofossils provide zonal assignments at sporadic intervals (Rahman, this volume). With this caveat in mind, some statements can be made with caution about time and intracore correlations.

The lower part of the upper Miocene sequence in the Japan Basin, approximately equivalent to the Denticulopsis dimorpha Diatom Zone (Koizumi, this volume) at Site 795 (Table 3), contains a distinctive assemblage of agglutinated and calcareous foraminifers. The agglutinated foraminifers include Cyclammina $\mathrm{cf}$. $C$. tani, $C y$ clammina pusilla, other Cyclammina species, more than six species of Haplophragmoides, more than three species of Ammodiscus, Trochammina spp., Martinottiella communis, Martinottiella nodulosa, and Eggerella sp. A. The calcareous foraminifers include Melonis pompilioides, Gyroidina soldanii, and other Gyroidina spp., Cibicides wuellerstorfi, Globobulimina auriculata, and Globigerina bulloides. 
Site 796 (Table 4), also from the Japan Basin, bears a similar agglutinated assemblage near its base. The base may be early late Miocene in age based on extrapolation of sedimentation rates from datums higher in the sequence (see Sediment Accumulation Rates section, Site 796 chapter in Tamaki, Pisciotto, Allan, et al. (1990). The calcareous assemblage at Site 796 contains Globobulimina auriculata, Gyroidina lamarckiana, Nonionella miocenica, Chilostomella oolina, Valvulineria spp., and Globigerina woodi among a few others, and looks different from the assemblage at Site 795. However, we caution that this conclusion is reached based on very few specimens.

The lower part of the upper Miocene sequence in the Yamato Basin is assigned to the Rouxia californica Diatom Zone at Site 797 (Koizumi, this volume). The zone at this site is truncated by a disconformity at its base. The interval (Table 5) includes one specimen of Cyclammina, four species of Haplophragmoides, Martinottiella communis, and Eggerella sp. A and is generally similar to the agglutinated assemblage of the Japan Basin. The calcareous foraminifers include Gyroidina lamarckiana, Pullenia salisburyi, Globobulimina auriculata, and other Globobulimina spp., Neogloboquadrina asanoi, and Neogloboquadrina himiensis. This list of species is different from those of the Japan Basin.

The lower part of the upper Miocene sequence at Site 794 is assigned to the Rouxia californica Diatom Zone through the Denticulopsis katayamae Diatom Zone (Koizumi, this volume) above the opal-A/opal-CT transition, which lies in Core 127-794A-31X. The base of the upper Miocene is placed near Sample 127-794A-14R-CC, based on extrapolation of sedimentation rates and the occurrence of the Discoaster hamatus Calcareous Nannofossil Zone in Sample 127-794B-12R-CC. Unfortunately, the foraminifers are too rare at Site 794 (Table 2) to compare with those in Site 797 (Table 5).

\section{Middle Miocene Foraminifers}

The deepest, foraminifer-bearing unit at Site 795 in the Japan Basin falls within the Denticulopsis praedimorpha and Denticulopsis hustedtii Diatom Zones (Koizumi, this volume). The latter zone lies near the base of the middle Miocene. The sequence (Table 3 ) bears Cyclammina $\mathrm{cf.} C$. tani and Cyclammina spp. with Eggerella sp. A, several species of Haplophragmoides, Martinottiella communis, Martinottiella nodulosa, and Spirosigmoilinella compressa. With the exception of the last species, the assemblage of agglutinated foraminifers is similar to the lower upper Miocene agglutinated assemblage at Sites 795 and 796. The calcareous foraminifers at Site 795 include Globobulimina auriculata, Cibicides wuellerstorfi, Elphidium subincertum, Gyroidina lamarckiana, Gyroidina orbicularis, Pullenia bulloides, and pieces of nodosarids and single specimens of Melonis pompilioides Nonionella spp. and other species. The most common planktonic species are Globigerina bulloides and Globigerinita glutinata, species commonly associated with cool surficial water masses.

\section{Middle to Lower (?) Miocene Foraminifers}

In the Yamato Basin, the age of foraminifer-bearing sediment near basement at Sites 794 and 797 appears coincident. The intervals at Sites 794 (Cores 127-794B-24R and -25R) and 797 (Cores 127-797B$47 \mathrm{X}$ through $-52 \mathrm{X}$ ) contain the calcareous nannofossil, Sphenolithus heteromorphus (Rahman, this volume), which ranges in age from 14.4 to 17.1 Ma (Berggren et al., 1985). Its range crosses the lower Miocene/middle Miocene boundary. The ranges of several planktonic foraminifers support this conclusion. At Site 797, Samples 127-797B52X-CC, and -797C-3R-CC, contain Catapsydrax parvulus, which ranges in age from Zone N7 to within Zone N15 (Kennett and Srinivasan, 1983), about 17.6 to 10.2 Ma (Berggren et al., 1985), and Globorotalia praescitula, which ranges in age from Zone N10 (Kennett and Srinivasan, 1983), about 14-15 Ma (Berggren et al., 1985), to $17.7 \mathrm{Ma}$ (Berggren et al., 1985). Additionally, Samples 127-797B51X-CC, -797B-52X-CC, and -794B-24R-CC, are dominated by the species Globoquadrina venezuelana of the warm subtropics, which suggests that the region was much warmer than assemblages higher in the sequences. The paleoenvironmental cooling occurred in the early middle Miocene (Saito, 1963; Chinzei, 1986).

Sites 794 and 797 have similar assemblages of foraminifers. Site 794 (Table 2) contains the agglutinated species Cyclammina cancellata, Cyclammina japonica, Cyclammina pusilla, Ammodiscus spp., more than four species of Haplophragmoides, Martinottiella communis, Martinottiella nodulosa, Spirosigmoilinella compressa, and Trochammina spp. The same agglutinated assemblage occurs at Site 797 (Table 5) with the additional species, Eggerella sp. A, four additional species of Haplophragmoides, and four additional species of Ammodiscus. Site 794 (Table 2) contains the calcareous species Chilostomella oolina, Gyroidina orbicularis, Gyroidina sp. A, Nonionella miocenica, and Oridorsalis tener. Site 797 (Table 5) contains the same calcareous species with Globobulimina auriculata and other Globobulimina species, Cibicides wuellerstorfi, Eilohedra rotunda, additional Chilostomella species, Pullenia bulloides, Valvulineria sadonica, and other species.

Most of the calcareous benthic species that occur at Sites 794 and 797 (Tables 2 and 5) of the Yamato Basin also occur at Site 795 (Table 3 ) of the Japan Basin. However, the Yamato Basin assemblage is much more diverse, and the agglutinated assemblages differ in their cyclamminid species. The planktonic specimens at Site 795 are few in abundance and poorly preserved in this interval so comparisons with those at Sites 794 and 797 are unclear. Further work is suggested to test if this interval of Site 795 is correlative with the middle to lower(?) Miocene intervals of the Yamato Basin.

It is interesting to note that the deepest foraminifer-bearing interval at Sites 794 and 797 (Tables 2 and 5) is overlain by a distinct barren interval as are the middle Miocene foraminifer-bearing interval at 795 (Table 3) and the upper Miocene foraminifer-bearing intervals at all four sites (Tables 2 through 5).

\section{Paleodepth Analysis}

Eighteen calcareous species of benthic foraminifers were selected as paleodepth indicators based on the work of Matoba (1977), Keller (1980), and Thompson (1980), in the Japan region and that of Ingle and Keller (1980), Culver and Buzas (1986), and Ingle et al. (1980) along the eastern rim of the Pacific Ocean. Occurrences of depth indicators were tabulated (Table 6) by basin and geologic series. Our inspection of Table 6 reveals the assumptions made about paleodepth indicators, and inspection of Tables 1 through 5 reveals the small sample size used to draw these conclusions. Sequences bearing benthic foraminifers have lain at lower middle bathyal depths since the lower to middle Miocene in the Yamato Basin in units that lie within $0.55 \mathrm{~m}$ (Site 794) and $0.65 \mathrm{~m}$ (Site 797) of igneous basement. The basal middle Miocene sequence at Site 795 was also deposited at lower middle bathyal depths in a foraminifer-bearing unit that lies 19 $\mathrm{m}$ above igneous basement.

\section{Range of the Low Oxygen Facies}

At all four sites the lower, middle, and lower upper Miocene sequences bear sedimentary structures suggestive of low oxygen. The structures consist of predominance of horizontally oriented burrows in bioturbated sections interspersed with laminated intervals where bioturbation is absent. The features are described in the barrel sheets of the Initial Reports volumes. The ranges of these structures correspond very well with the ranges of the agglutinated benthic foramini-feral assemblage bearing Cyclammina, Haplophragmoides, and Ammodiscus, an assemblage associated with low oxygen conditions (Matoba, 1984). At all four sites the association appears near the bases of the sedimentary sequences above igneous basement (Table 7). In the Japan Basin the association continues to the Neodenticula kamtschatica Diatom Zone in the upper upper Miocene of both sites. In the Yamato 
Table 2. Range chart of foraminifers from Site 794 arranged alphabetically within the taxonomic groups, agglutinated, miliolid, rotalid, and planktonic.

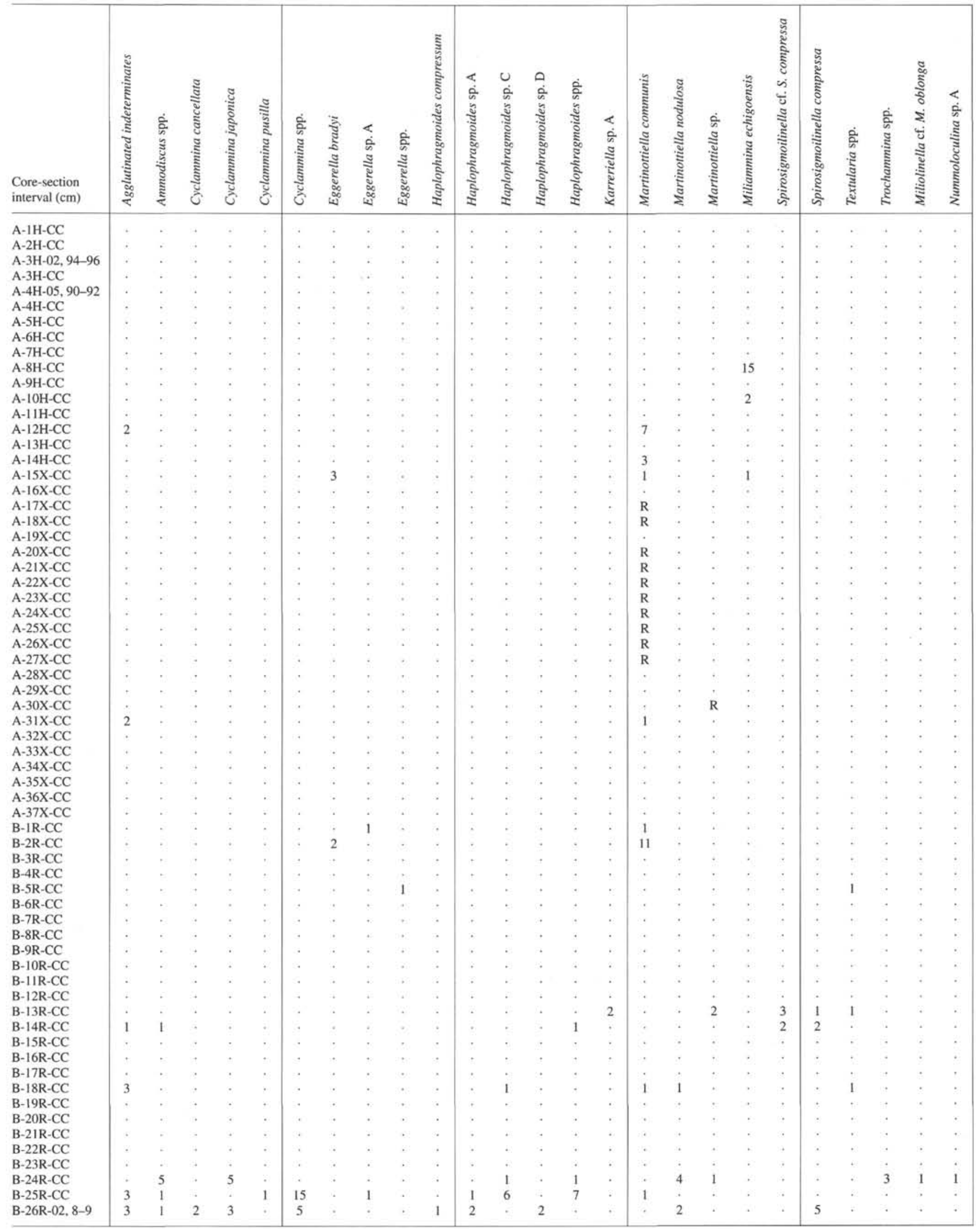




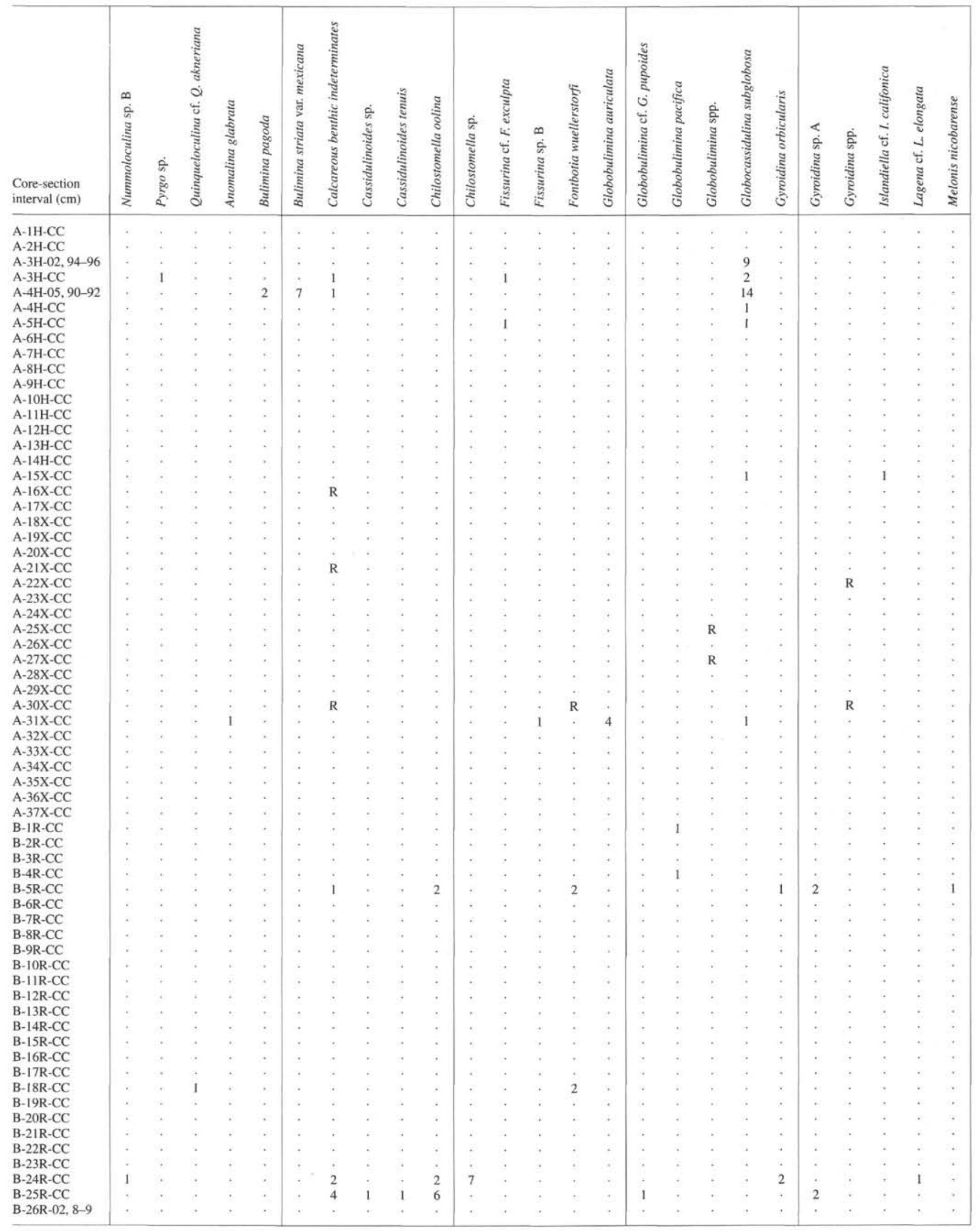




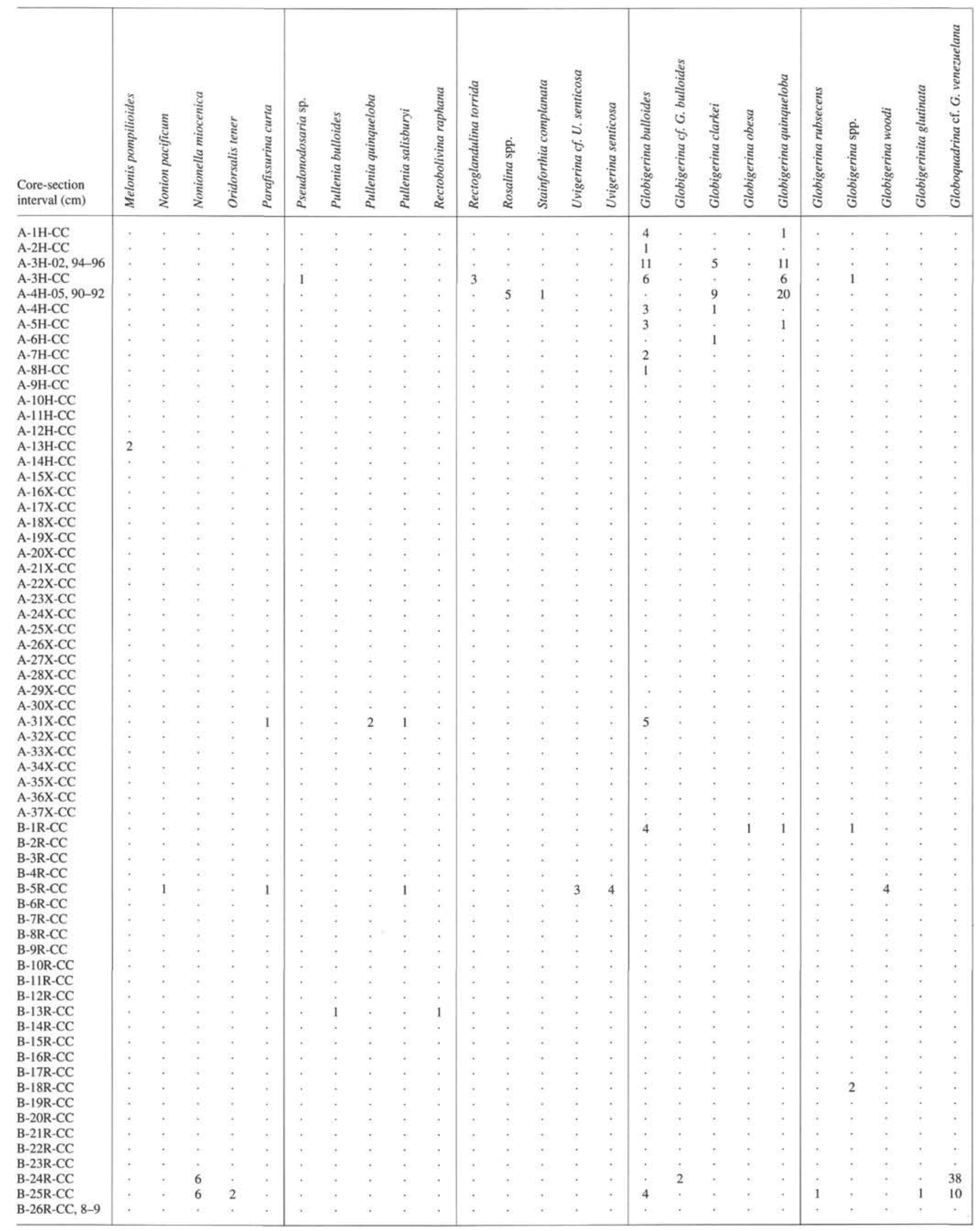


Table 2 (continued)

\begin{tabular}{|c|c|c|c|c|c|c|c|c|}
\hline $\begin{array}{l}\text { Core-section } \\
\text { interval }(\mathrm{cm})\end{array}$ & 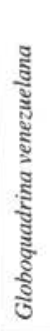 & 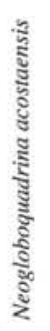 & 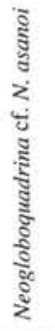 & 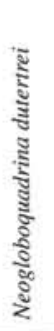 & 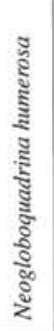 & 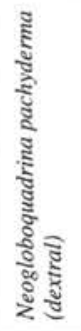 & 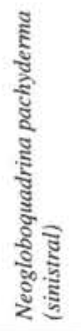 & 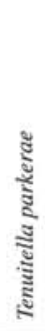 \\
\hline $\mathrm{A}-1 \mathrm{H}-\mathrm{CC}$ & . & . & . & . & . & 4 & 27 & . \\
\hline $\mathrm{A}-2 \mathrm{H}-\mathrm{CC}$ & . & . & . & . & . & $\therefore$ & 3 & 1 \\
\hline A-3H-02, 94-96 & . & . & . & . & , & $\therefore$ & 11 & . \\
\hline $\mathrm{A}-3 \mathrm{H}-\mathrm{CC}$ & . & . & + & . & . & 25 & 12 & . \\
\hline A- $4 \mathrm{H}-05,90-92$ & . & . & . & 7 & . & 28 & II & 13 \\
\hline $\mathrm{A}-4 \mathrm{H}-\mathrm{CC}$ & . & . & . & - & . & . & 12 & · \\
\hline A- $5 \mathrm{H}-\mathrm{CC}$ & . & . & $\cdot$ & - & . & 6 & 18 & . \\
\hline A- $6 \mathrm{H}-\mathrm{CC}$ & . & . & . &. & . & . & 1 & . \\
\hline A- $7 \mathrm{H}-\mathrm{CC}$ & . & . & . & . & . & . & . & . \\
\hline $\mathrm{A}-8 \mathrm{H}-\mathrm{CC}$ & . & . & . & . & . & 2 & 1 & . \\
\hline A-9H-CC & . & . & . & . & . & . & & . \\
\hline $\mathrm{A}-10 \mathrm{H}-\mathrm{CC}$ & . & . & . & . & . & . & . & . \\
\hline A- $11 \mathrm{H}-\mathrm{CC}$ & . & . & . & . & . & . & . & . \\
\hline $\mathrm{A}-12 \mathrm{H}-\mathrm{CC}$ & . & . & . & . & . & . & . & . \\
\hline $\mathrm{A}-13 \mathrm{H}-\mathrm{CC}$ & - & . & . & . & . & . & . & . \\
\hline $\mathrm{A}-14 \mathrm{H}-\mathrm{CC}$ & . & . & . & . & . & . & . & . \\
\hline A- $-15 X-C C$ & . & . & . & . & . & . & . & . \\
\hline A-16X-CC & & . & . & . & . & . & . & . \\
\hline A- $17 \mathrm{X}-\mathrm{CC}$ & . & . & . & . & . & . & . & . \\
\hline A- $18 \mathrm{X}-\mathrm{CC}$ & . & . & . & . & . & . & . & . \\
\hline A-19X-CC & . & . & . & . & . & , & . & . \\
\hline A-20X-CC & . & . & . & . & . & . & · & \\
\hline A- $21 X-C C$ & . & . & . & . & . & . & . & . \\
\hline $\mathrm{A}-22 \mathrm{X}-\mathrm{CC}$ & . & . & . & . & . & . & . & $\cdot$ \\
\hline A- $23 \mathrm{X}-\mathrm{CC}$ & - & . & & . & . & . & . & . \\
\hline A- $24 X-C C$ & , & . & . & . & . & . & . & . \\
\hline A- $25 \mathrm{X}-\mathrm{CC}$ & : & . & . & . & . & . & . & . \\
\hline A-26X-CC & . & . & . & . & . & . & . & . \\
\hline A-27X-CC & . & . & . & . & . & . & . & . \\
\hline A- $28 \mathrm{X}-\mathrm{CC}$ & . & . & . & . & . & . & . & . \\
\hline A-29X-CC & $\cdot$ & . & . & · & . & - & . & . \\
\hline A-30X-CC & . & . & . & . & . & . & . & . \\
\hline A-31X-CC & . & . & . & $\therefore$ & . & - & . & . \\
\hline A-32X-CC & . & . & . & . & . & . & . & . \\
\hline A-33X-CC & . & . & . & . & . & . & . & . \\
\hline A- $34 \mathrm{X}-\mathrm{CC}$ & . & . & . &. & . & . & . & . \\
\hline A-35X-CC & . & . & . & . & . & . & . & . \\
\hline A-36X-CC & . & . & . & . & . & . & . & . \\
\hline$A-37 X-C C$ & . & . & $\cdot$ & . & . & . & . & . \\
\hline B-IR-CC & . & . & 22 & $\cdot$ & . & . & 26 & . \\
\hline B-2R-CC & . & . & . & . & . & . & $\therefore$ & . \\
\hline B- 3 R-CC & . & . & & . & . & . & . & . \\
\hline B- $4 \mathrm{R}-\mathrm{CC}$ & . & . & . & . & . & . & . & . \\
\hline B-5R-CC & , & , & & & . & . & , & , \\
\hline B-6R-CC & . & . & . & . & . & . & & . \\
\hline B-7R-CC & . & . & & . & . & . & , & , \\
\hline B- $8 \mathrm{R}-\mathrm{CC}$ & . & . & & . & . & . & . & . \\
\hline B-9R-CC & . & . & & . & $\cdot$ & & . & . \\
\hline B-I0R-CC & . & . & & . & . & . & . & . \\
\hline B- 11 R-CC & . & . & & , & . & . & . & . \\
\hline B- $12 \mathrm{R}-\mathrm{CC}$ & $\therefore$ & . & . & . & . & . & . & . \\
\hline B-13R-CC & $\cdot$ & . & . & . & . & . & . & . \\
\hline B-14R-CC & . & . & . & . & . & . & . & . \\
\hline B- $15 \mathrm{R}-\mathrm{CC}$ & . & . & . & . & . & . & . & . \\
\hline B-16R-CC & . & . & . & . & . & . & . & . \\
\hline B-17R-CC & . & . & . & . & . & . & . & . \\
\hline B-18R-CC & . & : & . & . & - & . & . & . \\
\hline B-19R-CC & . & . & . & , & . & . & . & , \\
\hline B-20R-CC & . & . & . & . & . & . & 8. & . \\
\hline B-2IR-CC & . & . & . & . & . & . & . & . \\
\hline B-22R-CC & . & . & . & . & . & . & . & . \\
\hline B- $23 R-C C$ & . & . & . & . & . & . & . & . \\
\hline B-24R-CC & 2 & 1 & . & . & 6 & . & . & . \\
\hline B-25R-CC & . & . & . & . & . & . & : & . \\
\hline B-26R-CC, $8-9$ & . & . & . & . & . & · & . & . \\
\hline
\end{tabular}

Basin, the shallowest occurrence of the association lies within the range of calcareous nannofossil, Discoaster hamatus, at Site 794 of the lower upper Miocene and within the Rouxia californica Diatom Zone of the middle upper Miocene at Site 797.

\section{RESULTS OF HIGH-RESOLUTION PLIOCENE AND QUATERNARY BIOSTRATIGRAPHIC AND PALEOENVIRONMENTAL STUDY}

Planktonic foraminifers are limited to a few intervals at Sites 794 and 797 in the Yamato Basin. Of 325 samples examined, only 37 samples have more than 100 specimens, and 18 have fewer than 100 specimens picked from the entire sediment sample (Table 8).

There are 37 samples with planktonic foraminifer numbers greater than 40 tests/g (Fig. 1). Visual inspection of the sand-size fraction (Table 9) reveals that planktonic foraminifers are the most abundant constituents of the sand-size fraction in 29 of the 37 samples, including two samples that have sand-size gypsum and anhydrite crystals, which may have precipitated from the pore water upon drying in the laboratory and one sample dominated by authigenic pyrite with foraminifers as the next most common constituent. There is a clear relationship between above average sand content and large foramini-feral number. All samples with greater than 4,000 planktonic foraminifers/g have above average sand content, average being $2 \%$ for all 325 samples of the data set. There is some relationship between above average sand content and samples enriched with foraminifers. Sixteen samples are above average in sand content, and 11 are average or below average.

Eight of the 37 samples (Table 9) are dominated by mineral grains, authigenic pyrite with mineral grains, ash or pumice, or sponge spicules. Four of these also have an unusually large ratio of benthic to planktonic foraminifers, a condition that can indicate shallow source.

The coiling ratio of Neogloboquadrina pachyderma estimates relative paleotemperature of surface waters. All samples with 30 or more specimens of Neogloboquadrina pachyderma were considered (Table 10). At Site 794 (Fig. 2), all 8 samples have $90 \%$ or more sinistrally coiled forms. In contrast, Site 797 (Fig. 2) has 34 samples, 26 of which have $90 \%$ or more sinistrally coiled forms, 7 have between $80 \%$ and $89 \%$, and one sample, which lies within the Olduvai, has $63 \%$ sinistrally coiled forms.

Q-mode cluster analysis was used to distinguish planktonic foraminiferal assemblages in all samples in which 100 or more specimens were counted (Table 11). Four clusters were resolved (Fig. 3). Cluster 1 is composed of 11 samples, which are dominated $(>81 \%)$ by sinistral Neogloboquadrina pachyderma. The species has high production rates when the upper water column is cold, isothermal, and nutrient rich (Sautter and Thunell, 1989; Reynolds and Thunell, 1986). It is also associated with dissolution because sinistral Neogloboquadrina pachyderma is the most solution resistant of the high-latitude species (Coulbourn et al., 1980; Sautter and Thunell, 1989).

Cluster 2 is composed of 15 samples dominated by sinistral Neogloboquadrina pachyderma with significant amounts of other species, which subdivide the cluster into three groupings, a, b, and c. Cluster 2a includes accessory Globigerina quinqueloba, a species associated with the spring bloom, Cluster $2 \mathrm{~b}$ includes accessory Globigerina bulloides, a species associated with upwelling and a wide range of thermal conditions, and Cluster 2c includes accessory Globigerina bulloides and dextral Neogloboquadrina pachyderma, the latter of which is associated with the stratified upper water column of the temperate fall. The assemblage is consistent with one produced in subarctic waters during seasonal alternation of isothermal and stratified conditions. The fauna is, subsequently, only moderately biased by dissolution (Sautter and Thunell, 1989; 1991).

Cluster 3 is composed of four samples and consists of near equal proportions of sinistral Neogloboquadrina pachyderma and 
Table 3. Range chart of foraminifers from Site 795 arranged alphabetically within the taxonomic groups, agglutinated, miliolid, rotalid, and planktonic.

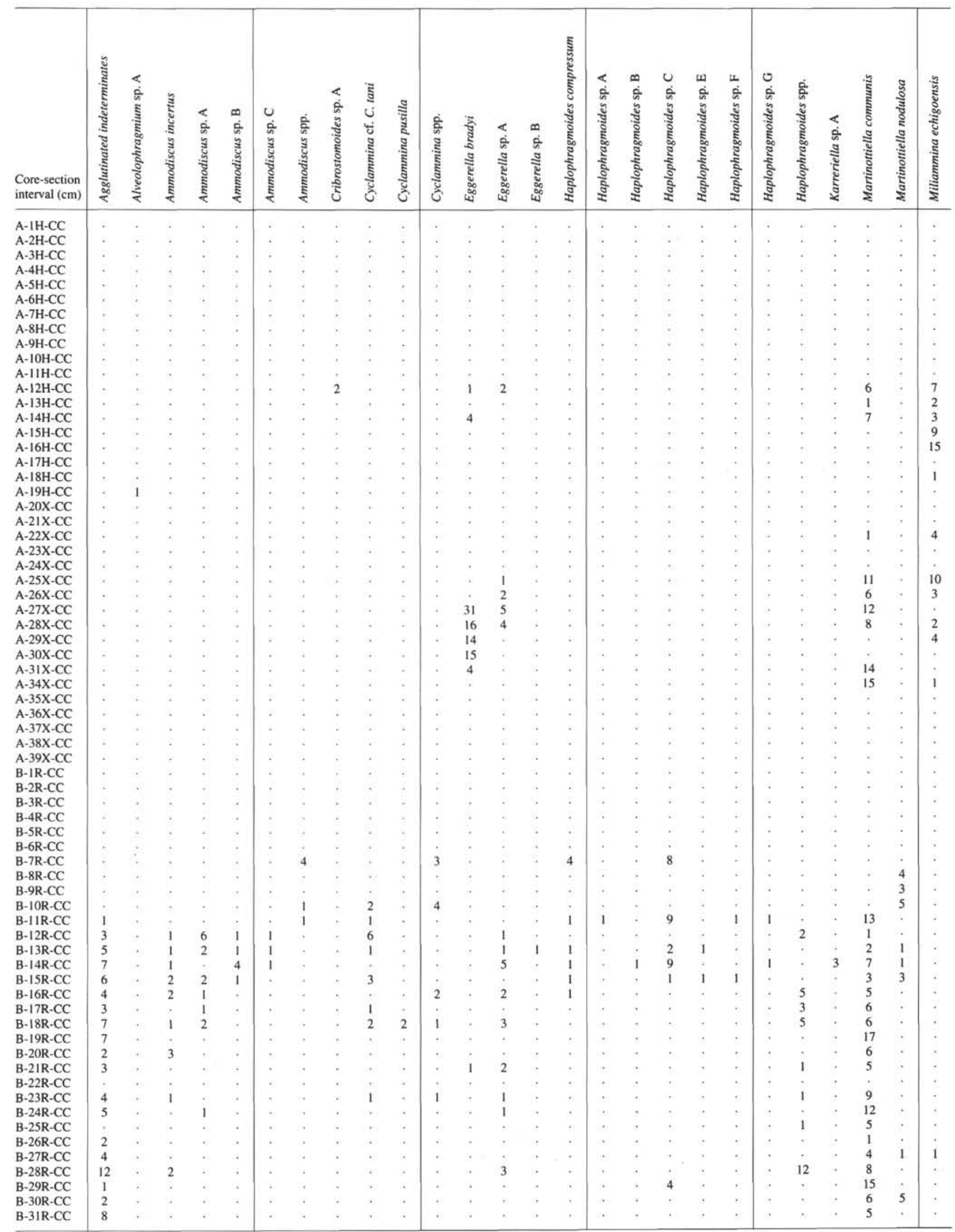


Table 3 (continued).

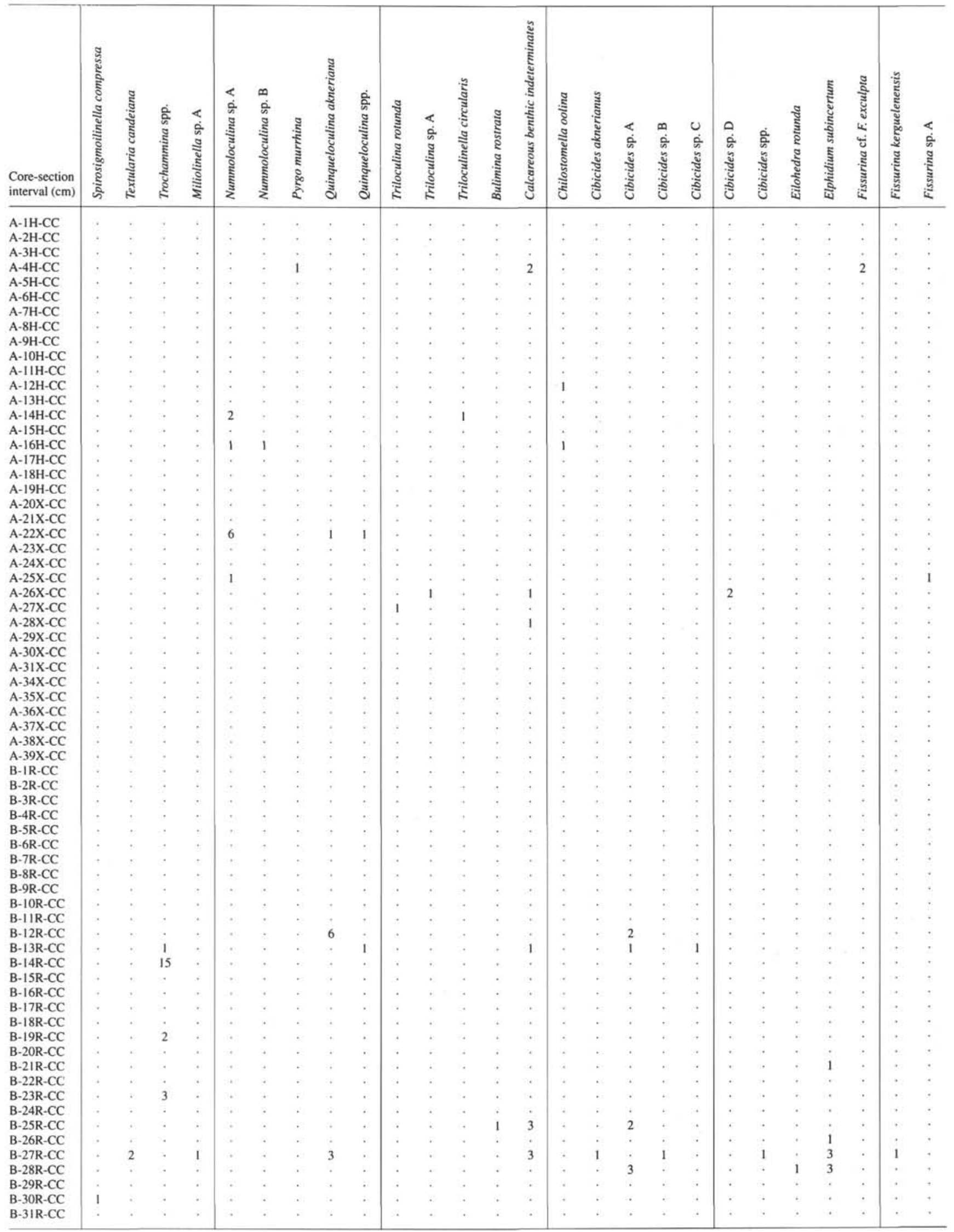


Table 3 (continued).

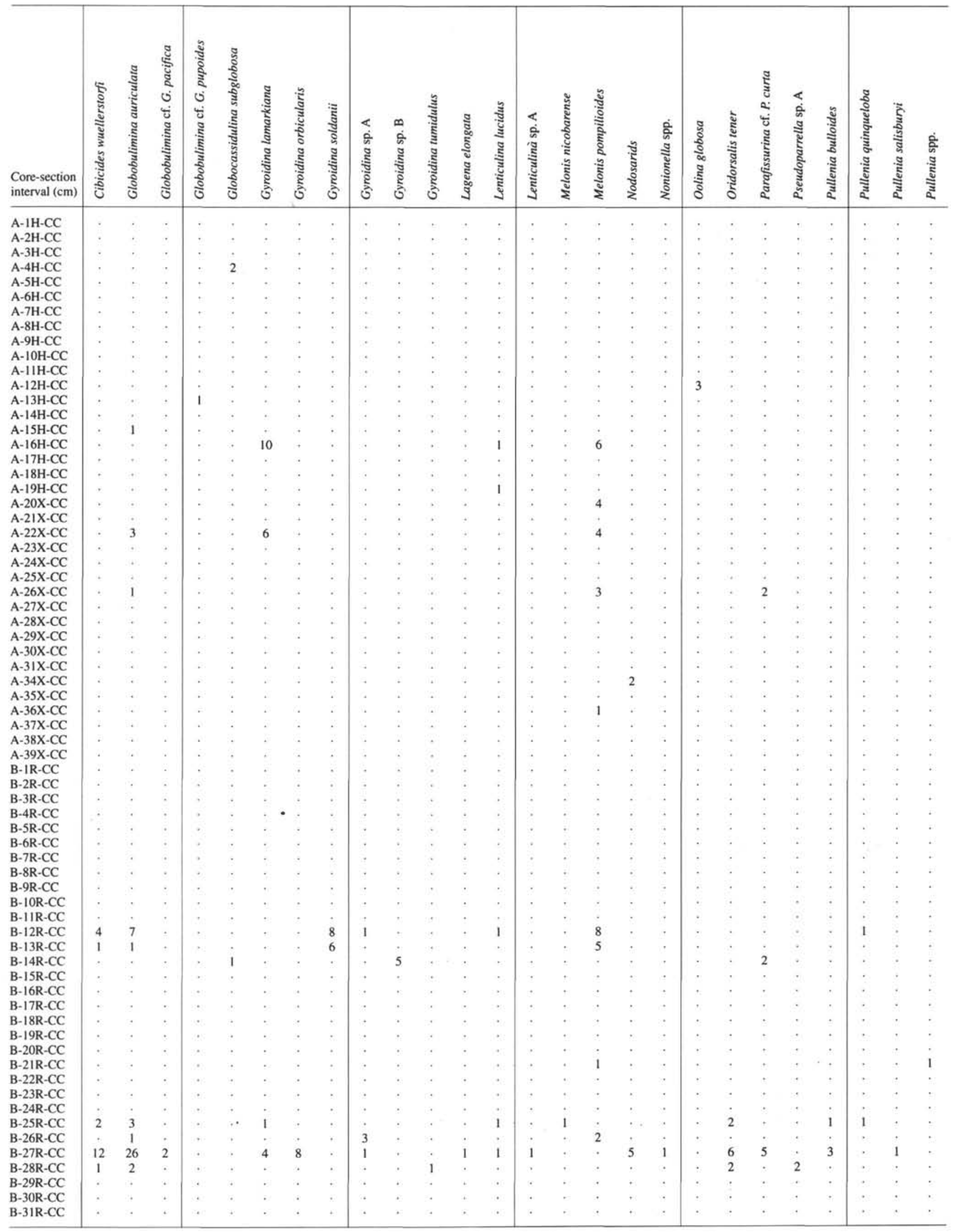


Table 3 (continued).

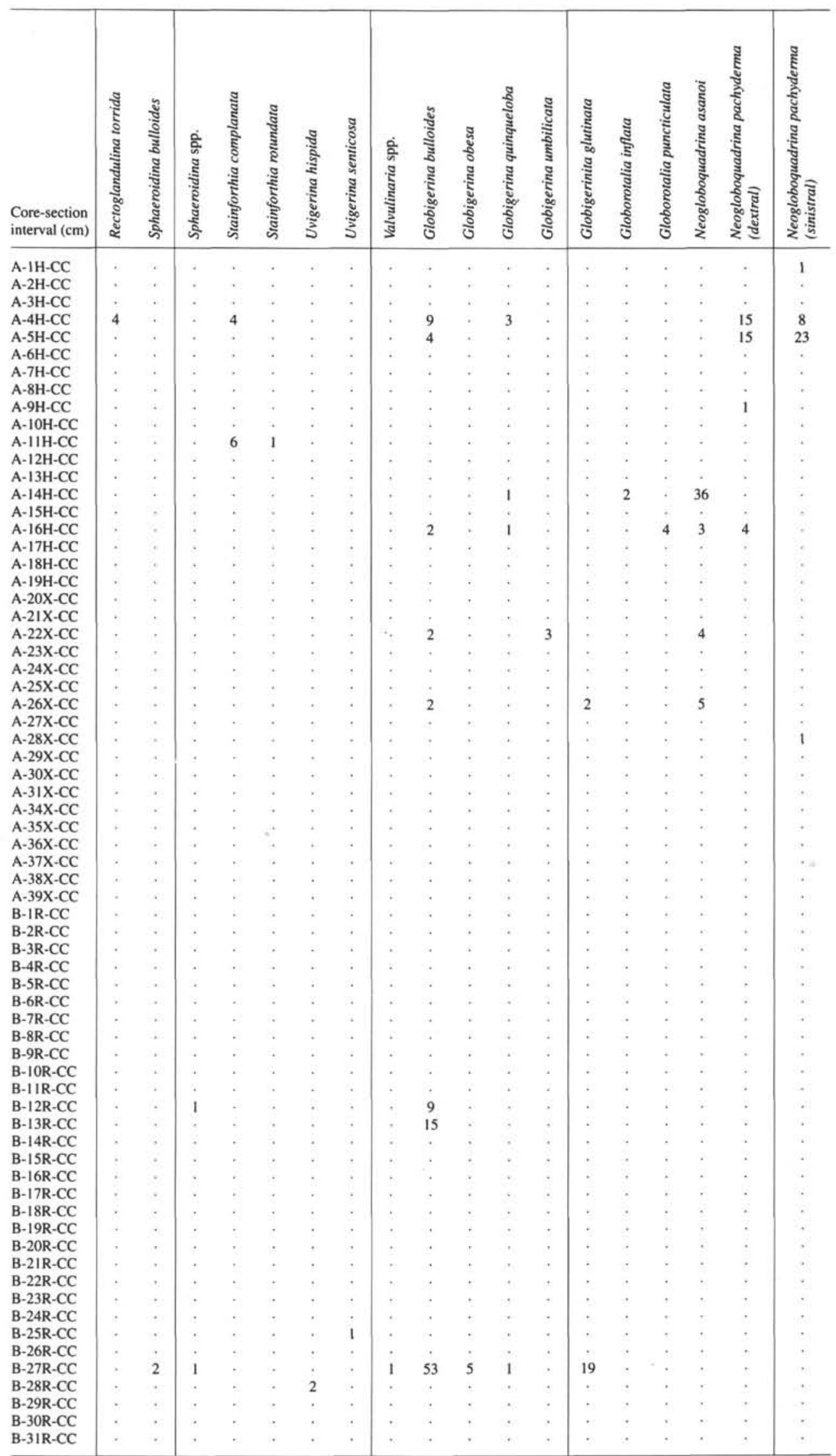


Table 4. Range chart of foraminifers from Site 796 arranged alphabetically within the taxonomic groups, agglutinated, milliolid, rotalid, and planktonic.

\begin{tabular}{|c|c|c|c|c|c|c|c|c|c|c|c|c|c|c|c|c|c|c|c|c|c|c|c|c|c|}
\hline $\begin{array}{l}\text { Core-section } \\
\text { interval (cm) }\end{array}$ & 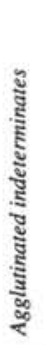 & $\begin{array}{l}\text { के } \\
\text { के } \\
\text { है } \\
\text { : } \\
\text { है } \\
\text { है }\end{array}$ & 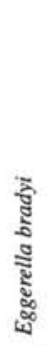 & 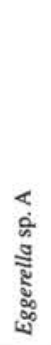 & 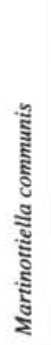 & 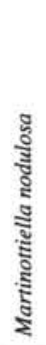 & 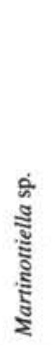 & 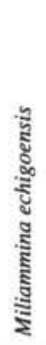 & 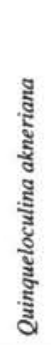 & 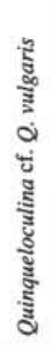 & 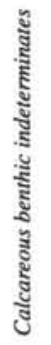 & 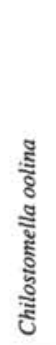 & 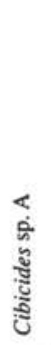 & 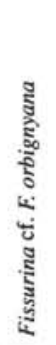 & 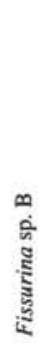 & 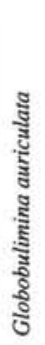 & 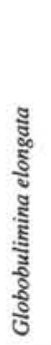 & 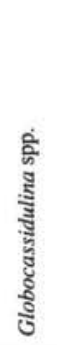 & 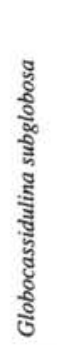 & 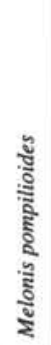 & 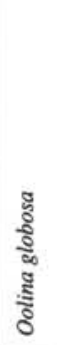 & 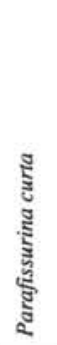 & 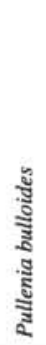 & 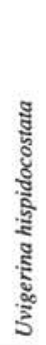 & 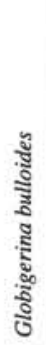 \\
\hline $\mathrm{A}-1 \mathrm{H}-\mathrm{CC}$ & 6 & . & . & . & . & . & . & . & . & 7 & . & & . & . & . & . & . & . & . & . & . & . & . & . & 99 \\
\hline A- $2 \mathrm{H}-\mathrm{CC}$ & . & & & & & . & . & . & & ! & & & & & . & . & . & . & . & . & & . & . & . & ? \\
\hline $\mathrm{A}-3 \mathrm{H}-\mathrm{CC}$ & . & . & . & & . & & & & . & . & . & & & . & , & & & & & & & . & . & . & 15 \\
\hline A- $4 \mathrm{H}-\mathrm{CC}$ & . & . & & & & . & & & . & . & . & . & . & & & & . & . & . & . & . & . & & & 15 \\
\hline A-5H-CC & . & . & . & & & . & & . & & & & . & . & . & . & . & . & , & & & . & . & . & . & . \\
\hline A- $6 \mathrm{H}-\mathrm{CC}$ & . & & . & . & & . & . & . & . & . & . & . & . & . & . & . & . & . & . & . & . & . & . & . & . \\
\hline A- $8 \mathrm{X}-\mathrm{CC}$ & . & . & . & . & & . & . & . & 4 & 8 & 1 & . & 1 & . & 2 & . & . & & . & . & . & . & . & . & 4 \\
\hline $\mathrm{A}-9 \mathrm{X}-\mathrm{CC}$ & . & . & 1 & . & & & . & & : & . & : & . & $\because$ & & & & . & . & & . & . & & & . & : \\
\hline A-10X-CC & . & . & . & . & . & . & & 8 & . & & . & . & . & . & & . & . & & & 1 & . & . & . & 1 & . \\
\hline$A-11 X-C C$ & & & . & & . & & & . & & & . & . & . & . & . & . & . & . & . & : & . & . & . & $\because$ & . \\
\hline$A-12 X-C C$ & . & . & & . & & . & . & 1 & . & . & & . & & & & & & & . & . & & . & & & . \\
\hline A-14X-CC & . & . & & & & . & . & & & & . & . & . & . & . & . & . & . & & . & . & . & . & . & . \\
\hline A-15X-CC & & & . & . & 10 & & & 3 & & & & . & . & . & . & . & . & . & & . & . & . & . & & . \\
\hline$A-16 X-C C$ & & & . & . & . & . & . & & . & . & & , & & & . & & & & . & . & & & & & . \\
\hline $\mathrm{A}-17 \mathrm{X}-\mathrm{CC}$ & , & . & . & 1 & 13 & . & & 1 & . & . & & . & & 1 & & 3 & . & . & . & . & . & 1 & & . & . \\
\hline$A-18 X-C C$ & , & . & 9 & : & . & . & . & : & . & . & & & . & : & . & . & . & . & . & & . & : & . & . & . \\
\hline A-19X-CC & . & . & . & . & & . & . & & & & & & . & . & . & . & . & . & . & . & . & . & . & . & . \\
\hline A-20X-CC & & . & . & . & . & . & . & & & & & & . & . & . & . & & & & . & . & . & & & . \\
\hline A-21X-CC & . & . & 2 & & & . & & 1 & & & & 8 & 1 & & . & 7 & 1 & 3 & 6 & . & 1 & . & . & . & . \\
\hline $\mathrm{A}-22 \mathrm{X}-\mathrm{CC}$ & . & . & . & . & 1 & . & . & & & . & & & $\therefore$ & . & . & . & : & . & . & & : & . & . & . & . \\
\hline A-23X-CC & . & . & . & . & . & . & . & & & & . & & . & . & . & . & . & . & . & . & . & . & . & & . \\
\hline A- $24 X-C C$ & & & . & . & 1 & . & . & & . & . & & . & & . & . & & & & & & . & & . & . & \\
\hline A- $25 \mathrm{X}-\mathrm{CC}$ & . & & . & & & . & . & & . & . & . & . & & & & . & . & & & . & . & . & . & . & \\
\hline$A-26 X-C C$ & & & & & & & . & & . & . & . & . & . & . & . & . & . & & & . & . & . & . & . & . \\
\hline $\mathrm{A}-27 \mathrm{X}-\mathrm{CC}$ & 3 & 3 & 1 & & 3 & 1 & 2 & 1 & . & . & 1 & . & . & . & . & . & . & . & & 3 & & & 1 & & . \\
\hline $\begin{array}{l}\text { Core-section } \\
\text { interval }(\mathrm{cm})\end{array}$ & 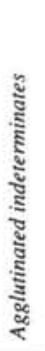 & 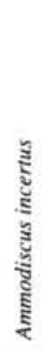 & 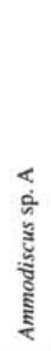 & 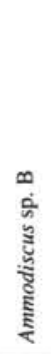 & 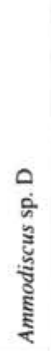 & 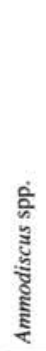 & 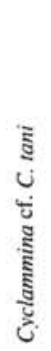 & 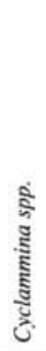 & 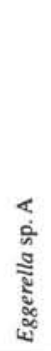 & 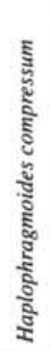 & 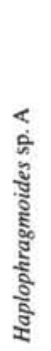 & 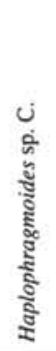 & 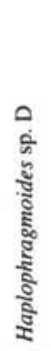 & 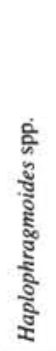 & 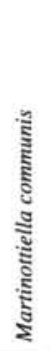 & 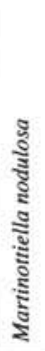 & 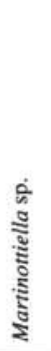 & 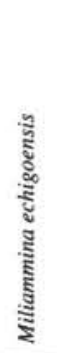 & 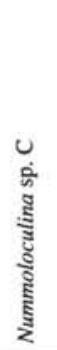 & 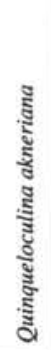 & 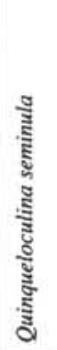 & 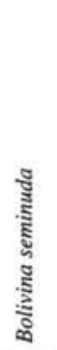 & 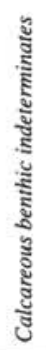 & 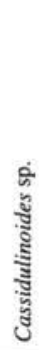 & 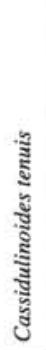 \\
\hline B-1R-CC & & & & & . & & . & . & . & . & . & . & . & . & . & . & . & & & . & . & & . & . & . \\
\hline B-2R-CC & & & & & . & . & & . & . & . & . & . & . & . & & . & & . & & . & . & . & . & & . \\
\hline B-3R-CC & & & . & & . & . & . & . & . & . & . & & & & & . & . & 2 & & . & . & . & 1 & 1 & 6 \\
\hline B- 4 R-CC & & & & & & $\therefore$ & . & & & . & 8 & . & . & . & . & . & . & 14 & & . & & . & . & & . \\
\hline B-5R-CC & & & & & . & . & & . & . & . & . & . & . & . & . & . & . & . & & . & . & . & . & & . \\
\hline B-6R-CC & . & & . & & . & . & & . & . & . & & & & & & . & . & & & & . & . & . & & . \\
\hline B-7R-CC & . & & , & . & . & & & . & 1 & , & & & & . & . & . & . & 2 & & . & . & . & 1 & & \\
\hline B-9R-CC & & & & & & & . & . & : & , & . & . & . & . & . & . & . & 27 & 2 & . & . & & 1 & . & \\
\hline B-10R-CC & & & & & & & & & . & . & . & & & & & & & & & & . & . & : & . & . \\
\hline B-IIR-CC & . & & . & . & . & . & . & . & . & . & & . & . & . & . & . & . & & & . & . & . & . & & . \\
\hline B-12R-CC & & & & & & & . & . & . & . & $\therefore$ & . & . & . & . & . & . & . & . & . & . & & . & . & \\
\hline B-13R-CC & . & & & & & . & . & & . & . & . & . & . & . & . & . & . & . & . & . & . & . & . & . & \\
\hline B-14R-CC & & & & & & & & & . & . & . & & & & & 1 & . & & & & . & . & . & . & . \\
\hline B-15R-CC & & & . & & . & 1 & & & & . & . & . & . & . & . & : & . & . & & . & . & . & & . & . \\
\hline B-16R-CC & . & . & . & & . & . & . & & . & . & . & . & . & . & . & . & . & & & . & . & + & & . & . \\
\hline B-17R-CC & & & & & & $\therefore$ & . & & & & . & . & . & . & . & & & . & . & . & . & . & . & . & \\
\hline B-18R-CC & & & & & & & & & . & & . & & & & 1 & . & . & & & & . & . & . & . & . \\
\hline B-19R-CC & . & . & & . & & . & . & & & . & . & . & . & 1 & 9 & 2 & & & . & 1 & 1 & : & 3 & . & . \\
\hline B-20R-CC & & & & & . & . & . & . & . & . & . & . & . & . & & . & . & . & & . & . & . & . & & . \\
\hline B- $24 \mathrm{R}-\mathrm{CC}$ & & & . & . & . & . & . & . & . & . & . & . & . & . & & . & . & . & & . & . & . & . & & . \\
\hline B- $28 \mathrm{R}-\mathrm{CC}$ & 5 & 2 & 4 & . & . & 1 & & 1 & . & 4 & . & 1 & & 4 & & 1 & 2 & & & . & . & . & . & . & . \\
\hline B-29R-CC & i & : & & . & & . & 3 & i & . & . & . & : & . & . & 1 & . & . & . & & . & . & . & & & . \\
\hline B-30R-CC & . & & & . & . & . & . & & . & . & . & . & . & . & : & . & . & & & . & . & . & . & & . \\
\hline B-3।R-CC & & & & 1 & . & . & & 1 & . & . & . & & & . & . & . & . & . & & . & . & . & . & . & . \\
\hline B- 32 R- $03,31-33$ & 2 & & & . & 1 & . & . & . & . & . & & 1 & & 2 & . & . & & & & . & . & . & . & : & . \\
\hline B-32R-04, $143-145$ & : & . & . & . & : & . & . & . & . & 3 & . & 15 & 1 & . & & . & . & . & & . & . & 1 & 8 & & \\
\hline B-32R-05, 24-26 & & & & & & & & 1 & . & . & & . & & . & & . & . & & & . & . & : & 5 & . & . \\
\hline B-33R-02, 34-36 & 3 & & . & . & . & . & . & & . & . & & . & . & . & . & . & . & . & . & . & . & & 3 & . & . \\
\hline
\end{tabular}


Table 4 (continued).

\begin{tabular}{|c|c|c|c|c|c|c|c|c|c|c|c|c|c|c|c|c|c|c|c|c|c|c|c|}
\hline $\begin{array}{l}\text { Core-section } \\
\text { interval }(\mathrm{cm})\end{array}$ & 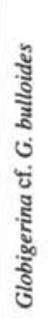 & 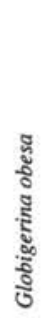 & 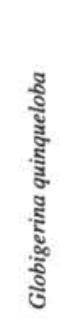 & 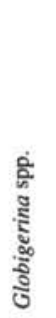 & 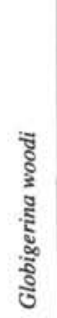 & 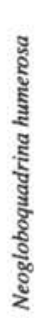 & 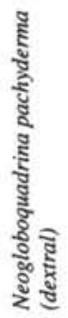 & 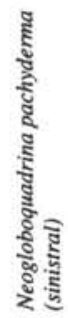 & & & & & & & & & & & & & & & \\
\hline A-1H-CC & . & 3 & . & . & . & . & 2 & 12 & & & & & & & & & & & & & & & \\
\hline A-2H-CC & & . & . & & . & . & . & ? & & & & & & & & & & & & & & & \\
\hline A-3H-CC & 19 & . & 1 & . & . & . & . & 7 & & & & & & & & & & & & & & & \\
\hline A- $4 \mathrm{H}-\mathrm{CC}$ & . & . & . & & . & & II & 35 & & & & & & & & & & & & & & & \\
\hline A-5H-CC & . & . & . & & & . & . & . & & & & & & & & & & & & & & & \\
\hline A-6H-CC & & & . & & . & . & . & & & & & & & & & & & & & & & & \\
\hline A- $8 \mathrm{X}-\mathrm{CC}$ & . & & 3 & . & . & 2 & 35 & & & & & & & & & & & & & & & & \\
\hline A-9X-CC & . & & . & . & & . & . & . & & & & & & & & & & & & & & & \\
\hline A-10X-CC & . & . & . & & & . & . & & & & & & & & & & & & & & & & \\
\hline A-11X-CC & . & & & & & . & . & & & & & & & & & & & & & & & & \\
\hline A-12X-CC & . & . & . & . & . & & & & & & & & & & & & & & & & & & \\
\hline$A-14 X-C C$ & . & . & . & . & & . & - & . & & & & & & & & & & & & & & & \\
\hline$A-15 X-C C$ & . & . & . & . & & & . & & & & & & & & & & & & & & & & \\
\hline A-16X-CC & . & . & & . & . & & . & . & & & & & & & & & & & & & & & \\
\hline $\mathrm{A}-17 \mathrm{X}-\mathrm{CC}$ & . & . & & & . & & & & & & & & & & & & & & & & & & \\
\hline A-18X-CC & . & . & . & & & & . & & & & & & & & & & & & & & & & \\
\hline A-19X-CC & . & & . & . & . & & . & & & & & & & & & & & & & & & & \\
\hline A-20X-CC & . & . & & & & , & & . & & & & & & & & & & & & & & & \\
\hline A-21X-CC & . & . & . & 1 & & & . & & & & & & & & & & & & & & & & \\
\hline A-22X-CC & & . & . & . & . & & & . & & & & & & & & & & & & & & & \\
\hline A-23X-CC & . & & & & & . & . & . & & & & & & & & & & & & & & & \\
\hline A-24X-CC & . & . & & . & . & & & & & & & & & & & & & & & & & & \\
\hline A-25X-CC & . & & . & . & & & . & . & & & & & & & & & & & & & & & \\
\hline A-26X-CC & . & & . & : & : & & . & . & & & & & & & & & & & & & & & \\
\hline A-27X-CC & . & & & 1 & 1 & . & . & . & & & & & & & & & & & & & & & \\
\hline $\begin{array}{l}\text { Core-section } \\
\text { interval }(\mathrm{cm})\end{array}$ & 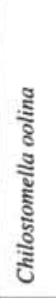 & 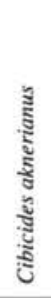 & 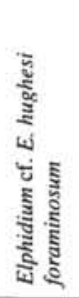 & 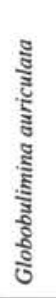 & 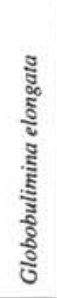 & 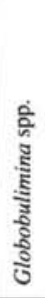 & 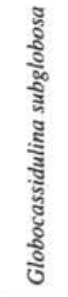 & 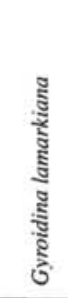 & 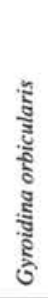 & 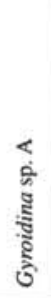 & 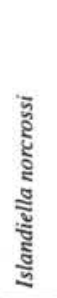 & 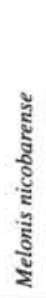 & 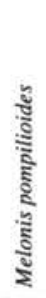 & $\begin{array}{l}\frac{1}{0} \\
\frac{5}{5} \\
\frac{5}{2} \\
2\end{array}$ & 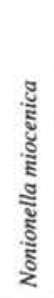 & 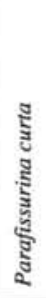 & 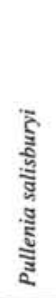 & 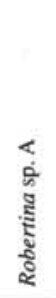 & 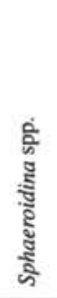 & $\begin{array}{l}\text { ปूँ } \\
\text { हूँ } \\
\text { है } \\
\text { हूँ } \\
\text { हू } \\
\text { जू }\end{array}$ & 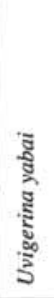 & 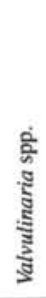 & 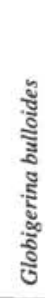 \\
\hline B-1R-CC & & & . & . & . & , & & . & . & . & & & . & . & & & . & . & . & & . & . & 1 \\
\hline B-2R-CC & & . & . & . & . & . & & . & & & . & . & . & . & . & . & . & . & & . & . & . & 1 \\
\hline B-3R-CC & & . & & & & & . & . & . & . & . & . & . & . & . & . & . & . & . & & . & . & 2 \\
\hline B-4R-CC & & & & . & . & . & . & . & . & . & . & & . & . & . & . & . & . & . & & . & & . \\
\hline B-5R-CC & & & . & . & . & . & & . & . & . & & . & . & . & & . & . & . & . & . & . & & . \\
\hline B-6R-CC & & & & & & . & & . & & . & . & . & . & & . & . & . & & . & . & . & . & . \\
\hline B-7R-CC & . & 1 & 3 & 1 & & . & & . & & . & . & 1 & 1 & . & , & . & . & . & 1 & . & 4 & . & . \\
\hline B-9R-CC & & & . & . & . & . & . & & . & . & . & & . & . & . & & . & . & . & & . & . & \\
\hline B-10R-CC & & & . & . & . & . & & . & & & 1 & . & . & . & . & . & . & & & . & . & . & . \\
\hline B-IIR-CC & & & . & . & . & & . & . & & . & . & . & . & & & & . & . & . & & & . & . \\
\hline B-12R-CC & & & & . & . & & & . & . & . & . & & . & & & . & . & . & . & & . & . & . \\
\hline B-13R-CC & & & . & . & . & & . & . & . & . & & & . & . & & & . & . & . & & . & . & . \\
\hline B-14R-CC & & . & . & . & . & & & . & & . & . & . & . & . & . & . & . & & . & . & . & . & . \\
\hline B-15R-CC & & & & & & . & $\therefore$ & . & . & . & . & & & . & . & . & . & . & . & & & . & . \\
\hline B-16R-CC & & . & & & . & & & . & . & . & . & & . & . & . & & . & . & . & . & . & . & \\
\hline B-17R-CC & & & . & . & . & & . & . & & . & & . & . & & . & . & . & . & & . & . & . & . \\
\hline B-18R-CC & & . & . & . & . & & . & . & & . & . & . & . & & . & . & & . & . & . & & . & . \\
\hline B-19R-CC & & . & & & . & . & 8 & . & 1 & . & & & . & . & . & 1 & . & . & . & & . & . & . \\
\hline B-20R-CC & & & & . & . & . & . & & : & . & . & . & . & . & . & : & . & . & . & . & . & . & . \\
\hline B- $24 R-C C$ & & & . & . & . & . & & . & . & & . & . & . & . & . & . & . & & & . & . & . & . \\
\hline B-28R-CC & & & & & & . & & . & & . & & . & & & . & . & . & . & . & . & . & . & . \\
\hline B-29R-CC & & & . & . & . & & . & & . & . & & . & . & . & . & & . & . & & . & . & . & \\
\hline B-30R-CC & & & . & . & $\cdot$ & & . & . & . & . & . & . & . & & & . & . & . & . & & . & . & . \\
\hline B-3IR-CC & & . & . & . & . & & . & . & & . & . & . & . & . & . & $\therefore$ & . & . & . & & & . & . \\
\hline B-32R-03, 31-33 & & & & & & & . & . & . & 7 & & & . & . & . & & . & . & . & & . & . & . \\
\hline B-32R-04, $143-145$ & 8 & & . & 2 & . & & . & . & . & 34 & & . & . & 1 & 14 & . & . & 2 & & 1 & . & 1 & . \\
\hline B-32R-05, 24-26 & 5 & & . & : & 1 & 2 & . & 1 & & 24 & . & . & . & : & 2 & . & & 1 & . & & . & 5 & . \\
\hline B-33R-02, 34-36 & 16 & & . & . & . & 11 & . & 51 & . & . & . & . & & . & 3 & . & 1 & . & . & & . & 2 & . \\
\hline
\end{tabular}


Table 4 (continued).

\begin{tabular}{|c|c|c|c|c|c|c|c|}
\hline $\begin{array}{l}\text { Core-section } \\
\text { interval }(\mathrm{cm})\end{array}$ & & & & & & & \\
\hline $\begin{array}{l}\text { A-1H-CC } \\
\text { A-2H-CC } \\
\text { A-3H-CC } \\
\text { A-4H-CC } \\
\text { A-5H-CC } \\
\text { A-6H-CC } \\
\text { A-8X-CC } \\
\text { A-9X-CC } \\
\text { A-10X-CC } \\
\text { A-11X-CC } \\
\text { A-12X-CC } \\
\text { A-14X-CC } \\
\text { A-15X-CC } \\
\text { A-16X-CC } \\
\text { A-17X-CC } \\
\text { A-18X-CC } \\
\text { A-19X-CC } \\
\text { A-20X-CC } \\
\text { A-21X-CC } \\
\text { A-22X-CC } \\
\text { A-23X-CC } \\
\text { A-24X-CC } \\
\text { A-25X-CC } \\
\text { A-26X-CC } \\
\text { A-27X-CC }\end{array}$ & & & & & & & \\
\hline $\begin{array}{l}\text { Core-section } \\
\text { interval }(\mathrm{cm})\end{array}$ & 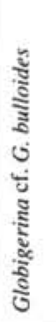 & 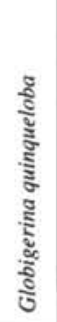 & 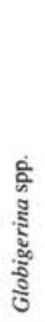 & 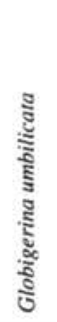 & 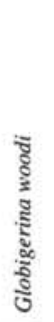 & 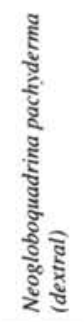 & 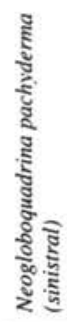 \\
\hline B-IR-CC & & & & . & & & \\
\hline $\mathrm{B}-2 \mathrm{R}-\mathrm{CC}$ & & . & & . & & . & 1 \\
\hline B-3R-CC & 1 & 1 & 1 & 4 & & 12 & 6 \\
\hline$B-4 R \cdot C C$ & 1 & . & . & & . & 7 & 15 \\
\hline B-5R-CC & & & . & . & 1 & . & . \\
\hline B-6R-CC & . & & : & & $\therefore$ & . & . \\
\hline B-7R-CC & & & & & 1 & . & . \\
\hline B-9R-CC & . & . & . & . & 1 & . & . \\
\hline B-10R-CC & . & & & . & . & . & . \\
\hline B-IIR-CC & . & & . & & & & . \\
\hline B-12R-CC & . & . & . & & & & . \\
\hline B-13R-CC & . & . & . & . & & . & \\
\hline B-14R-CC & . & & . & . & & , & . \\
\hline B-15R-CC & . & . & . & . & . & & . \\
\hline B-16R-CC & . & . & & & & . & \\
\hline B-17R-CC & & & . & . & & . & \\
\hline B-18R-CC & . & & . & & & . & . \\
\hline B-19R-CC & . & . & . & & 1 & & . \\
\hline B-20R-CC & . & . & . & . & : & . & \\
\hline B- $24 R-C C$ & . & . & . & . & & . & \\
\hline B-28R-CC & & & & & & . & . \\
\hline B-29R-CC & . & . & . & . & . & . & . \\
\hline B-30R-CC & . & . & . & . & . & . & \\
\hline B-31R-CC & & . & . & . & & . & . \\
\hline B-32R-03, 31-33 & . & . & . & . & 1 & . & - \\
\hline B-32R-04, 143-145 & . & . & 5 & . & 2 & . & . \\
\hline B-32R-05, 24-26 & . & & & . & 1 & . & . \\
\hline B-33R-02, 34-36 & & & 6 & . & . & . & . \\
\hline
\end{tabular}

Globigerina bulloides. This association is found in present-day surficial sediments in the subarctic North Pacific Ocean at water depths from 3,300 to 4,300 $\mathrm{m}$ (Coulbourn et al., 1980). The assemblage is produced under the same production conditions as that of Cluster 2, but it is subsequently more strongly dissolved (Sautter and Thunell, 1989).

Three samples cluster above the cutoff value and are not sufficiently similar to be regarded a cluster. They are most similar to cluster 3. One sample bears a high relative frequency of a species rare in all other samples, Tenuitella parkerae. One sample contains near equal proportions of sinistral Neogloboquadrina pachyderma, Globigerina bulloides, and Globigerina quinqueloba with accessory dextral Neogloboquadrina pachyderma. The last sample is dominated by sinistral Neogloboquadrina pachyderma with significant but small proportions of Globigerina bulloides, Tenuitella parkerae, and Turborotalita humilis.

Cluster 4 is composed of three samples and is dominated by Globigerina bulloides. These samples are the most different from all other samples of the data subset. Globigerina bulloides production is accelerated during upwelling events (Thunell, pers. comm., 1990) but thrives in a wide range of environments and is not limited to upwelling habitats especially in temperate and subpolar waters (Sautter and Thunell, 1991).

The Quaternary lithologic sequences at all four sites bear distinctive alternations in color between dark chocolate brown, medium brown, and light putty gray. It is interesting to note that each planktonic cluster has some samples from each color interval (Table 11).

\section{DISCUSSION}

\section{Foraminifer Biostratigraphy}

The benthic foraminifer sequences described above can be assigned to the biostratigraphy of Matsunaga (1963) (Table 12), which was further described by Matoba (1984) and Matoba et al. (1990) for the oil fields of Northern Japan. The low abundance of foraminifers at Sites 794 through 797 does not justify comparison to the zonules defined by Matsunaga (1963).

\section{Globorotalia cf. G. fohsi Zone}

The middle or lower(?) Miocene foraminifer-bearing sequences at Sites 794 and 797 are tentatively assigned to the Globorotalia cf. $G$. fohsi Zone. The absence of the subtropical marker species, Globorotalia $\mathrm{cf}$. $G$. fohsi, precludes certain assignment, but the occurrence of Globorotalia praescitula supports the conclusion. The dominance of a subtropical planktonic foraminifer assemblage suggests that the Japan Sea was open to southern waters during deposition of the sequences, a situation expected within the zone. The benthic species listed are similar to the bathyal fauna of the Nishikurosawa stage described by Matoba (1984). Some planktonic species listed (Tables 4 and 5) are reported from the Nishikurosawa by Saito and Maiya (1973), although some taxonomic questions need resolution.

\section{Spirosigmoilinella compressa Zone}

The barren interval above the Globorotalia cf. G. fohsi Zone at Sites 794 and 797, the middle Miocene sequence at Site 795, and the upper Miocene sequences at all four sites including the barren interval within the Neodenticula kamtschatica Diatom Zone fit the definition of the zone. At all four sites, the lower Pliocene, foraminifer-bearing intervals below the first occurrence of Miliammina echigoensis are also assigned to this zone. Evidence from the planktonic foraminifer fauna support the assignment. The cold-water fauna confirms that surficial waters had become isolated from subtropical waters that flowed on the Pacific margin of the Japanese islands during deposition of these sequences. 


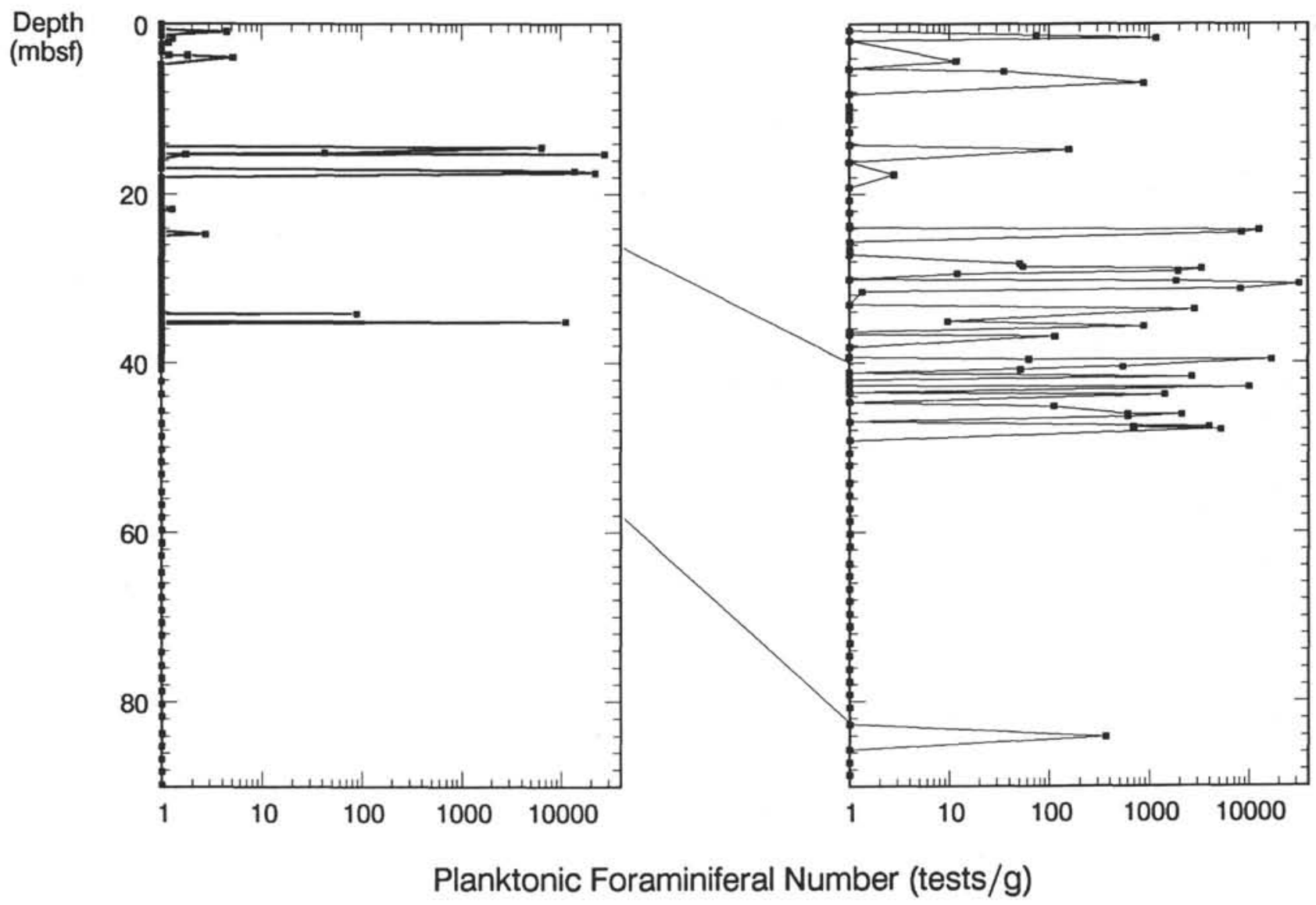

Figure 1. Planktonic foraminifer number (number of tests/g of sediment) plotted with depth in core in Hole 794A and Hole 797B. All samples plotted on the $y$ axis are barren of planktonic foraminifers. The two lines connecting the graphs denote the Brunhes/Matuyama Boundary and the top of the

\section{Miliammina echigoensis Zone}

The Pliocene sequence that includes the range of Miliammina echigoensis is assigned to the Miliammina echigoensis Zone. The interval extends from the Thalassiosira oestrupii Zone at its base to the uppermost Neodenticula koizumii Diatom Zone.

The Quaternary interval is not similar to the zonation described by Matsunaga. The species list, however, is similar to those reported by Ingle (1975), Matoba (1984), Ujiié et al. (1983), and Kato (1989).

\section{Event: Deepening of the Nascent Backarc Basin}

The Yamato Basin has lain at lower middle bathyal depths since the middle or early(?) Miocene, and the Japan Basin has been at a similar depth at least since the middle Miocene (Table 6). Others (Guber and Merrill, 1983; Matoba, 1984) have similarly concluded that some sections in the Hokuroku District and other regions surrounding Akita were bathyal and lower bathyal in depth as early as the early to middle Miocene. Neritic, upper bathyal, and middle bathyal elements are mixed with lower bathyal species in all of the Miocene sequences. Further, there is no clear change in paleodepth in either the Yamato or Japan basins from the middle or lower(?) Miocene sequences through the Pliocene section. Apparently, subsidence of the basin occurred very rapidly after emplacement of the igneous "basement" or during emplacement of the igneous "basement." No foraminifers at Sites 794,795 , or 797 are preserved that record the subsidence in sediments above the igneous basement or in sediment intercalated amid the igneous basement units.

\section{Event: Early Middle Miocene Cooling}

The accuracy of the date of change from subtropical to cold surficial water in the Japan Sea is not increased by this data set and is essentially no different from that cited by Matoba et al. (1990). It occurs above the base of the Sphenolithus heteromorphus Zone and within or below the base of the Denticulopsis praedimorpha Diatom Zone of the middle middle Miocene based on paleoenvironmental interpretation of the planktonic foraminifers at Sites 794, 795, and 797 (Tables 2, 3, and 5). Samples in the Sphenolithus heteromorphus Zone at Site 794 and 797 contain subtropical species. Two samples, 127-795B-27R-CC, and 127-795B-13R-CC, contain no subtropical species and are dominated by Globigerina bulloides and Globigerina cf. G. bulloides, which are characteristic of transitional faunas. An assemblage that includes high-latitude Neogloboquadrina pachyderma appears at Site 794 within or below the Denticulopsis katayamae Diatom Zone of the late Miocene. Like land-based sections, the ODP sequences are plagued with barren intervals and unconformities.

\section{Event: Oxygenation of Stagnant Miocene Deep Water}

Matoba (1984) and others relate the range of agglutinated assemblages in the Miocene to low-oxygen conditions. The ranges of Cyclammina-Ammodiscus-Haplophragmoides assemblages in the Miocene correspond with the occurrence of distinctive intervals dominated by horizontal burrows (Table 7) described by the shipboard sedimentologists (Tamaki, Pisciotto, Allan, et al., 1990). The uppermost occurrence of the association lies in the upper upper Miocene 
Table 5. Range chart of foraminifers from Site 797 arranged alphabetically within the taxonomic groups, agglutinated, milliolid, rotalid, and planktonic.

\begin{tabular}{|c|c|c|c|c|c|c|c|c|c|c|c|c|c|c|c|c|c|c|c|c|c|c|c|c|c|}
\hline $\begin{array}{l}\text { Core-section } \\
\text { interval }(\mathrm{cm})\end{array}$ & 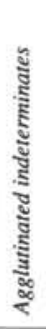 & 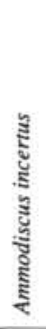 & 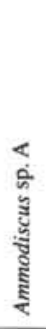 & 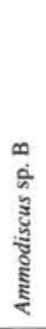 & 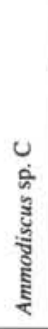 & 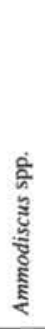 & $\begin{array}{l}< \\
\text { की } \\
\text { पूँ } \\
\text { हूँ } \\
\text { हैं } \\
\text { है } \\
\text { है }\end{array}$ & 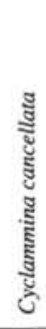 & 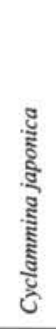 & 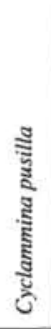 & 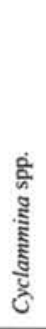 & 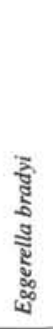 & 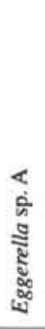 & 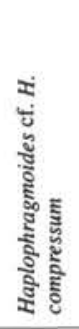 & 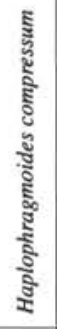 & 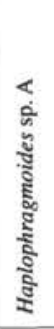 & 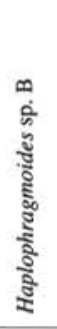 & 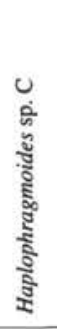 & 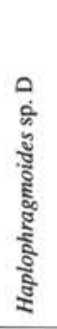 & 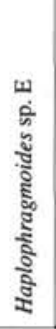 & 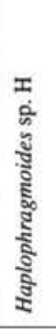 & 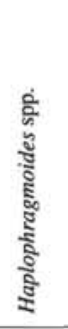 & 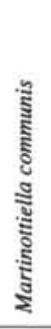 & 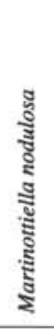 & 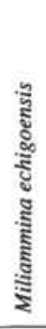 \\
\hline $\mathrm{A}-\mathrm{IH}-\mathrm{CC}$ & . & & . & . & . & . & . & . & & . & & . & . & , & . & . & . & & . & . & . & . & & . & . \\
\hline B-1H-CC & . & . & . & . & & . & & . & . & . & . & & . & . & . & . & . & . & . & . & . & & & & . \\
\hline B- $2 \mathrm{H}-\mathrm{CC}$ & . & . & . & & & . & . & . & & . & . & & & . & . & . & . & . & . & . & & & . & . & . \\
\hline B- $3 \mathrm{H}-\mathrm{CC}$ & & . & . & & & & & . & & & & & & . & . & . & : & . & . & . & . & . & . & . & . \\
\hline B- $4 \mathrm{H}-\mathrm{CC}$ & . & . & . & . & . & . & . & . & & & & . & & & . & . & & . & & . & . & . & . & . & . \\
\hline B-5H-CC & & & & & . & & & . & & . & & . & . & . & . & . & . & . & . & . & . & . & & & . \\
\hline B-6H-CC & . & . & . & & & . & . & & & & & . & . & . & . & . & . & . & . & . & . & & & . & . \\
\hline B- $7 \mathrm{H}-\mathrm{CC}$ & . & . & . & & & . & . & & & & & & & & . & . & & . & . & . & & . & & . & . \\
\hline B- $8 \mathrm{H}-\mathrm{CC}$ & . & . & . & & & . & & & & & & & & . & . & . & & . & . & . & . & . & & . & . \\
\hline B-9H-CC & & & & & & & & . & & . & & & & . & . & . & . & . & . & . & . & & & . & . \\
\hline B-10H-CC & . & . & . & & . & . & . & . & & . & & & & & . & . & . & . & . & . & . & . & 1 & . & . \\
\hline B-11H-CC & . & . & . & & . & . & . & . & & & & . & 1 & & . & & & & & . & . & & 1 & . & . \\
\hline B-12H-CC & . & . & . & & & . & & & & & & & . & . & . & & & & . & . & & . & & . & 1 \\
\hline B-13H-CC & . & . & . & & & . & & & & & & & . & . & . & . & . & . & . & . & & & & . & 2 \\
\hline B-14H-CC & & & & & & & . & . & & . & & . & . & . & . & . & . & . & . & . & . & . & . & . & 7 \\
\hline B-15H-CC & . & . & . & - & . & . & . & . & & . & & & 3 & & . & . & & . & & . & . & . & 13 & . & 5 \\
\hline B-16H-CC & . & . & . & . & & . & . & . & & & & & . & & . & . & & & . & . & . & . & 4 & . & . \\
\hline B-17H-CC & . & . & . & & . & . & . & & & & & . & 1 & . & . & & & . & . & . & . & & & . & . \\
\hline B- $18 \mathrm{H}-\mathrm{CC}$ & & & & & & & & & & & & 7 & . & & . & . & . & . & . & . & . & . & & . & 13 \\
\hline B-19H-CC & 2 & . & . & & . & & . & & & . & . & . & 2 & & & . & . & . & & . & & . & 5 & . & : \\
\hline B-20X-CC & 1 & . & . & . & . & . & . & . & & & . & 3 & . & & . & . & & & & . & . & & 1 & & 6 \\
\hline B-2IX-CC & . & & . & . & . & . & . & . & & . & . & . & 4 & & . & & . & . & . & . & . & . & 12 & . & 2 \\
\hline B-22X-CC & . & & & & & . & . & . & . & . & . & 1 & 2 & & . & . & . & . & . & . & . & & 12 & . & \\
\hline B-23X-CC & & & & & & & & & & & & . & & & . & . & . & . & & . & . & & & + & . \\
\hline B-24X-CC & 1 & . & . & . & . & & 1 & . & & . & . & . & . & & . & . & & & & . & . & . & 1 & & . \\
\hline B- $25 \mathrm{X}-\mathrm{CC}$ & . & . & . & . & . & & : & . & & & . & . & & . & . & & & . & . & . & . & . & & . & . \\
\hline B-26X-CC & & & . & & . & . & & . & . & & . & . & & & . & . & . & . & . & . & . & . & 6 & . & \\
\hline B-27X-CC & & & & . & & & & . & & . & & . & & & . & . & & & . & . & . & . & & . & . \\
\hline B- $28 \mathrm{X}-\mathrm{CC}$ & . & . & & & & & 1 & & . & . & . & . & & & . & . & & & . & . & . & $\therefore$ & 8 & . & . \\
\hline B-29X-CC & . & . & . & . & . & . & $\therefore$ & . & & . & & . & . & & . & . & . & . & . & . & . & 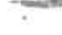 & . & & . \\
\hline B-30X-CC & . & . & . & . & . & . & . & . & & . & . & . & & & . & & . & . & . & . & . & & . & . & \\
\hline B-3IX-CC & . & & . & & . & . & . & . & & . & . & & . & . & . & . & . & . & . & . & & . & . & & . \\
\hline B-32X-CC & 1 & & & & & . & & . & & & . & . & & . & . & . & . & . & & . & . & . & : & . & . \\
\hline B-33X-CC & 2 & . & & . & + & & . & . & . & . & 1 & . & . & 2 & & & & 3 & 1 & 6 & . & . & 7 & . & \\
\hline B- 34 X-CC & . & . & . & . & . & & . & . & . & . & & . & & . & . & . & . & . & . & . & . & . & & . & \\
\hline B- $35 \mathrm{X}-\mathrm{CC}$ & . & . & . & . & . & . & . & . & & . & & & 1 & & & . & . & 1 & 2 & . & & & 3 & . & . \\
\hline B-36X-CC & 3 & & & & & & & & & & . & . & i & & . & . & & 1 & . & . & . & . & 4 & . & . \\
\hline B-37X-CC & 4 & . & & . & & 1 & . & . & & . & & & 2 & & & & . & & . & . & . & & 14 & . & \\
\hline B- $38 \mathrm{X}-\mathrm{CC}$ & : & . & . & . & . & $\therefore$ & . & . & . & . & & . & . & . & . & . & . & & . & . & . & . & & . & . \\
\hline B-39X-CC & . & . & . & . & . & . & . & . & . & . & & & . & . & & & . & & & . & & & & . & . \\
\hline B- $40 \mathrm{X}-\mathrm{CC}$ & . & . & . & . & . & . & . & . & & . & & . & . & & & & . & . & & . & . & . & & & . \\
\hline B-41X-CC & & & & & & . & & & & & & . & . & & . & . & & & . & . & . & & & . & . \\
\hline B- $42 \mathrm{X}-\mathrm{CC}$ & . & . & & . & . & . & . & & . & . & . & . & & & . & . & . & . & . & . & . & . & . & . & . \\
\hline B- $43 \mathrm{X}-\mathrm{CC}$ & . & . & + & , & . & . & . & . & . & . & . & . & & . & . & . & & & & . & & & . & . & . \\
\hline B- $44 \mathrm{X}-\mathrm{CC}$ & & & & & & . & & & & & & & & & & . & . & . & & . & . & . & . & . & . \\
\hline B- $45 \mathrm{X}-\mathrm{CC}$ & & & & , & & & . & & . & . & . & . & & & . & . & . & . & . & . & . & . & . & . & . \\
\hline B- $46 \mathrm{X}-\mathrm{CC}$ & . & . & . & . & + & . & . & . & . & . & . & & & & . & . & . & . & & . & & . & . & . & . \\
\hline B- $47 \mathrm{X}-\mathrm{CC}$ & 1 & . & . & & . & . & & & & & . & . & . & . & & & . & . & . & . & . & . & . & . & . \\
\hline B- $48 \mathrm{X}-\mathrm{CC}$ & 2 & & & & & & . & & . & 2 & . & . & . & . & & . & . & 2 & , & . & . & . & 9 & . & . \\
\hline B-49X-CC & & . & & . & . & . & . & . & . & . & . & . & . & . & . & & . & & . & . & . & & . & . & . \\
\hline B-50X-CC & . & . & . & . & . & . & . & . & . & . & & . & & . & & & & & . & . & - & & : & . & \\
\hline B- $51 X-C C$ & . & . & . & . & . & . & . & . & . & . & & 2 & . & 1 & & . & . & . & . & . & & & 3 & 1 & . \\
\hline B-52X-CC & 2 & 1 & 2 & . & . & , & & . & & 7 & & 2 & . & : & 6 & 3 & . & 3 & 1 & . & . & . & 4 & . & . \\
\hline C-IR-CC & . & & & . & & & & & . & . & . & . & . & . & . & . & & & . & . & . & . & & . & - \\
\hline C-2R-CC & 2 & . & . & & & . & . & & . & . & . & 1 & & 5 & . & 2 & 1 & & . & . & & 1 & . & . & . \\
\hline C-3R-CC & : & : & $\therefore$ & & . & . & . & . & & 4 & & & . & . & & & 2 & $\therefore$ & & . & . & . & & : & . \\
\hline C- $6 \mathrm{R}-\mathrm{CC}$ & 3 & 1 & 4 & 1 & . & . & . & 1 & & 3 & . & . & . & . & . & 3 & 16 & 3 & . & . & 5 & . & 8 & 1 & . \\
\hline C-7R-CC & 6 & 1 & 3 & . & 1 & . & . & . & 1 & 6 & 3 & . & & & . & . & 11 & 6 & & . & 4 & 1 & . & . & . \\
\hline C-8R-02, 36-38 & . & 2 & 3 & 2 & . & . & . & 1 & . & 5 & 1 & . & 2 & & 5 & . & 8 & 2 & . & . & & 8 & 6 & 1 & \\
\hline C-18R-01, 88-90 & . & . & . & & . & . & . & . & . & . & & & & . & & & . & & & . & . & . & . & . & \\
\hline C- $20 \mathrm{R}-02,42-44$ & & . & . & & . & . & . & +. & & & & . & . & . & & & , & . & . & . & . & . & . & . & . \\
\hline$C-22 R-C C$ & . & . & & . & & . & . & . & . & . & . & . & . & . & . & & . & & . & . & . & . & . & . & . \\
\hline C-23R-CC & . & & & & . & . & & & . & & . & . & & & . & . & & & . & . & & & . & . & . \\
\hline C- $25 \mathrm{R}-06,33-35$ & & . & & & & & . & . & . & . & & & & . & & . & . & & & . & . & . & . & . & . \\
\hline C-27R-CC & & . & & . & . & & . & . & & . & . & . & . & . & . & . & . & & & . & . & . & . & - & . \\
\hline C- $33 \mathrm{R}-03,42-44$ & & . & . & . & & . & . & & . & . & . & . & & . & . & . & & & . & . & . & . & . & . & . \\
\hline C-34R-CC & . & & . & & & . & & . & . & . & . & . & & . & & . & . & . & . & $\cdot$ & $\cdot$ & $\cdot$ & $\cdot$ & $\cdot$ & $\cdot$ \\
\hline
\end{tabular}


Table 5 (continued).

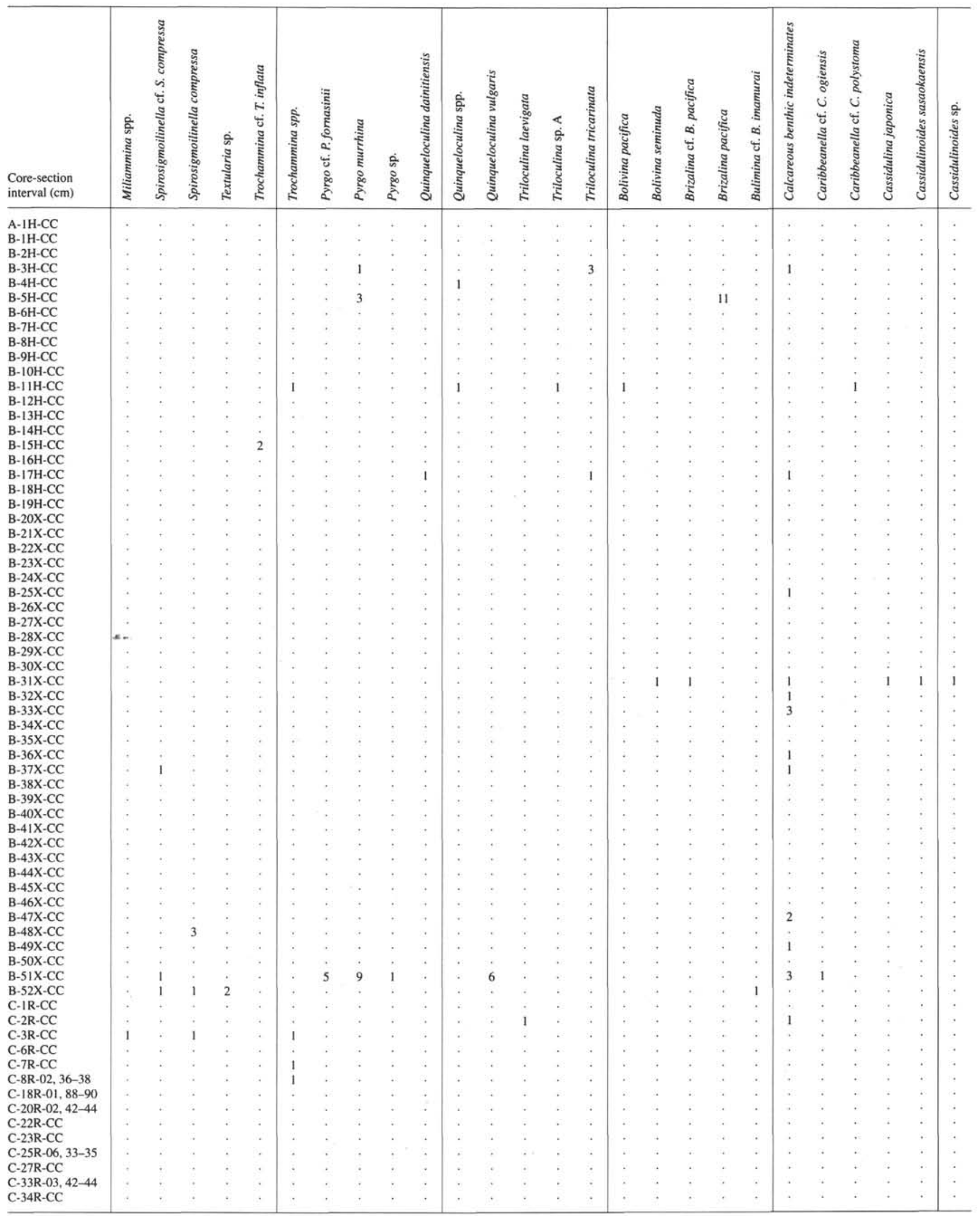


Table 5 (continued).

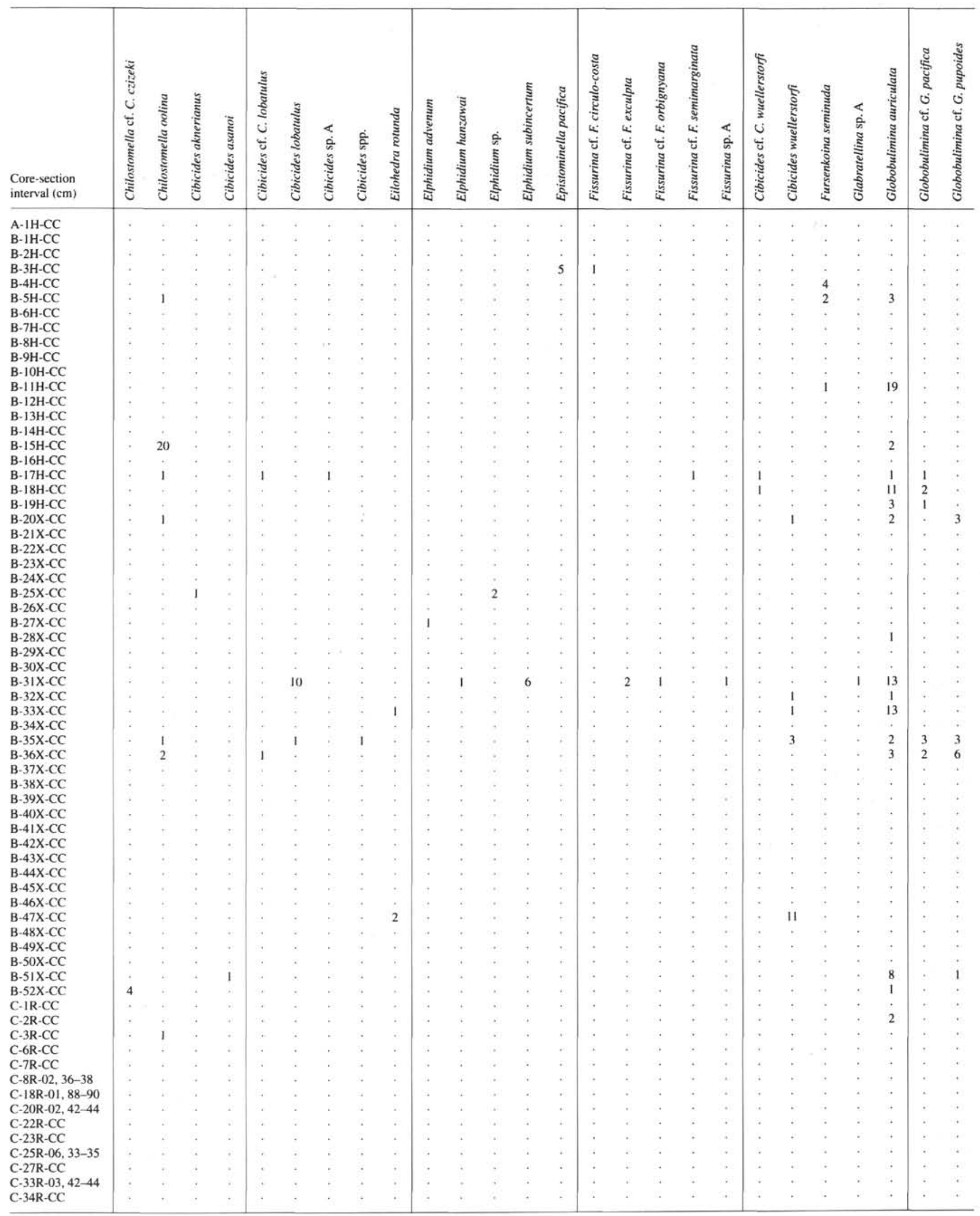


Table 5 (continued).

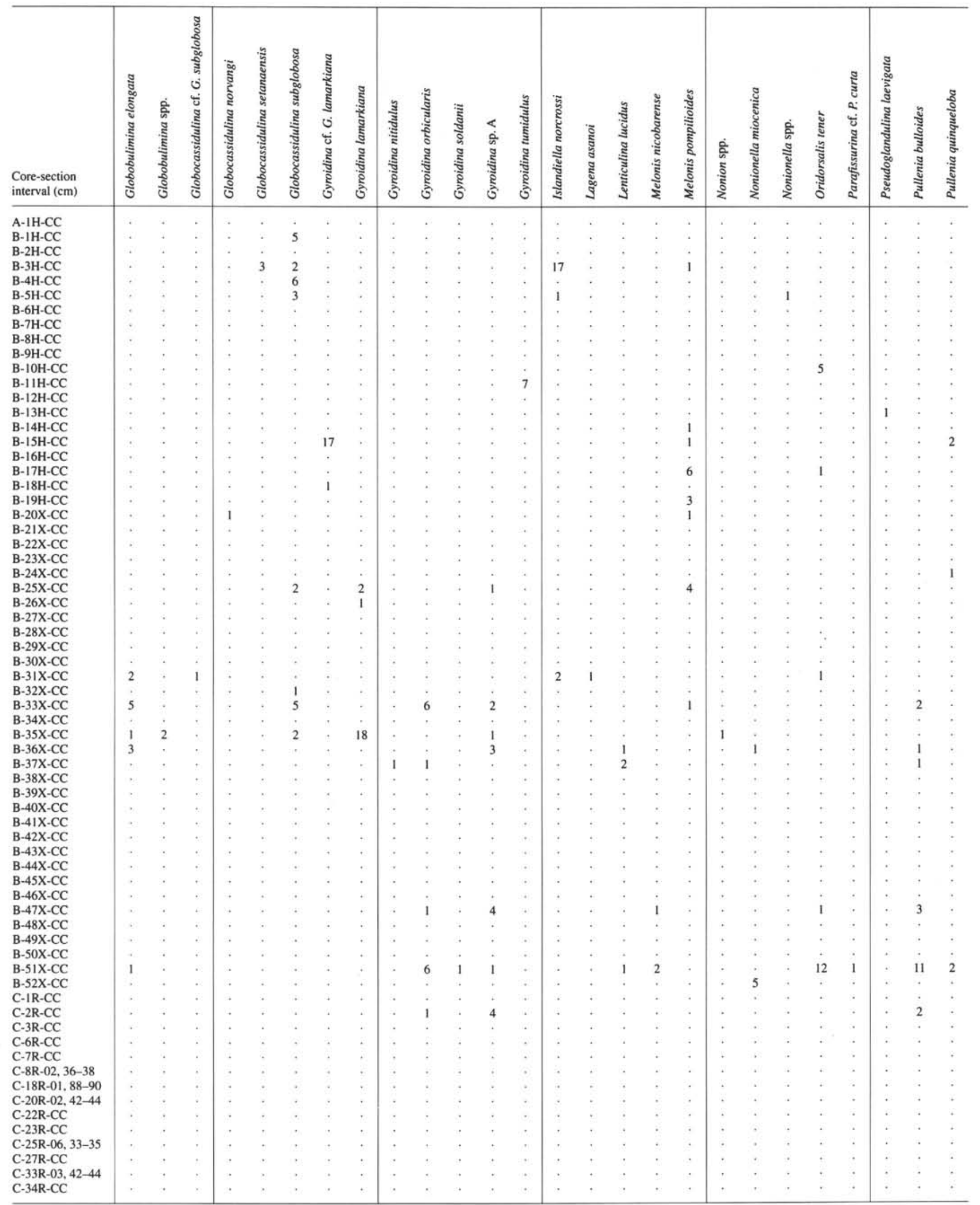


Table 5 (continued).

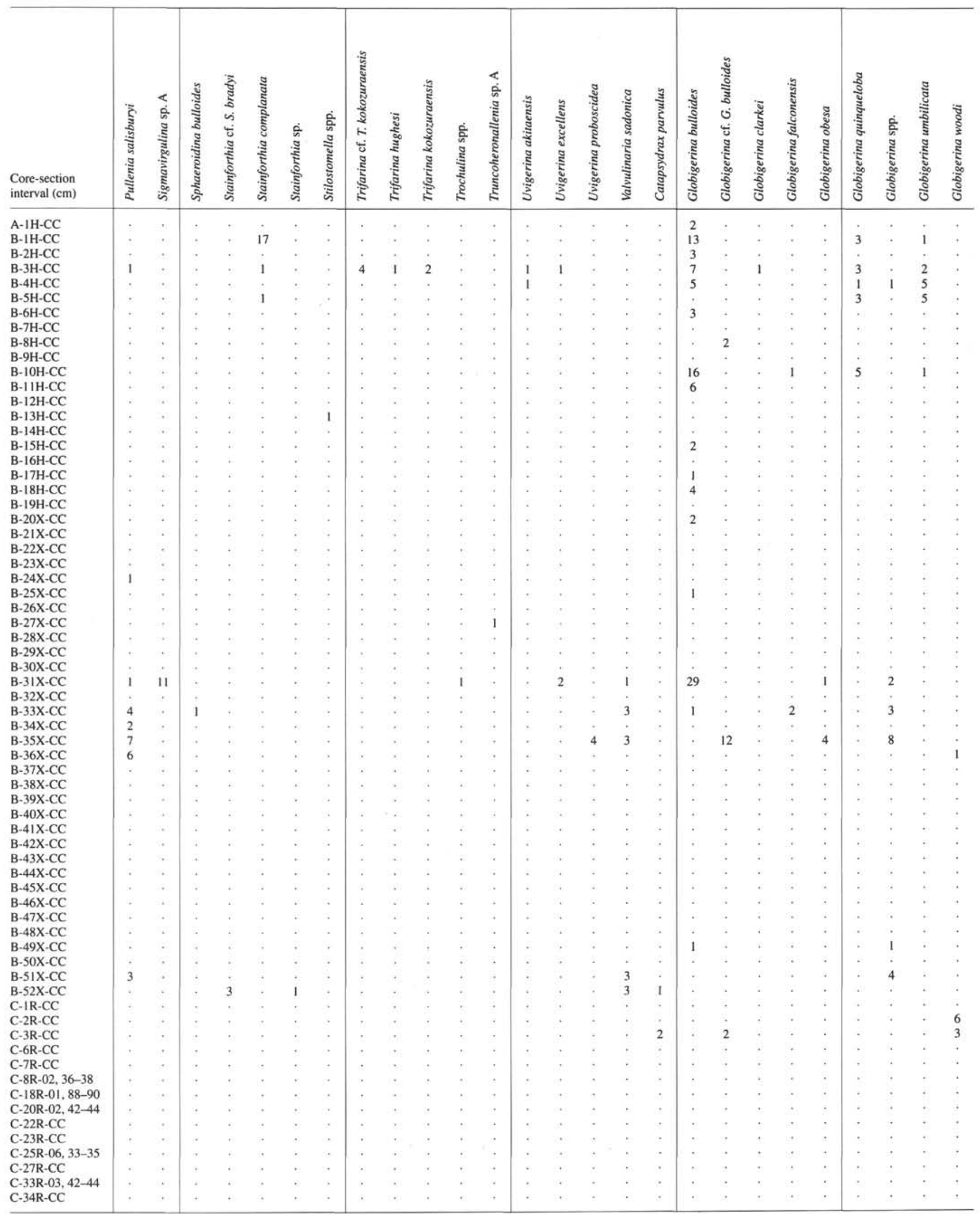


Table 5 (continued).

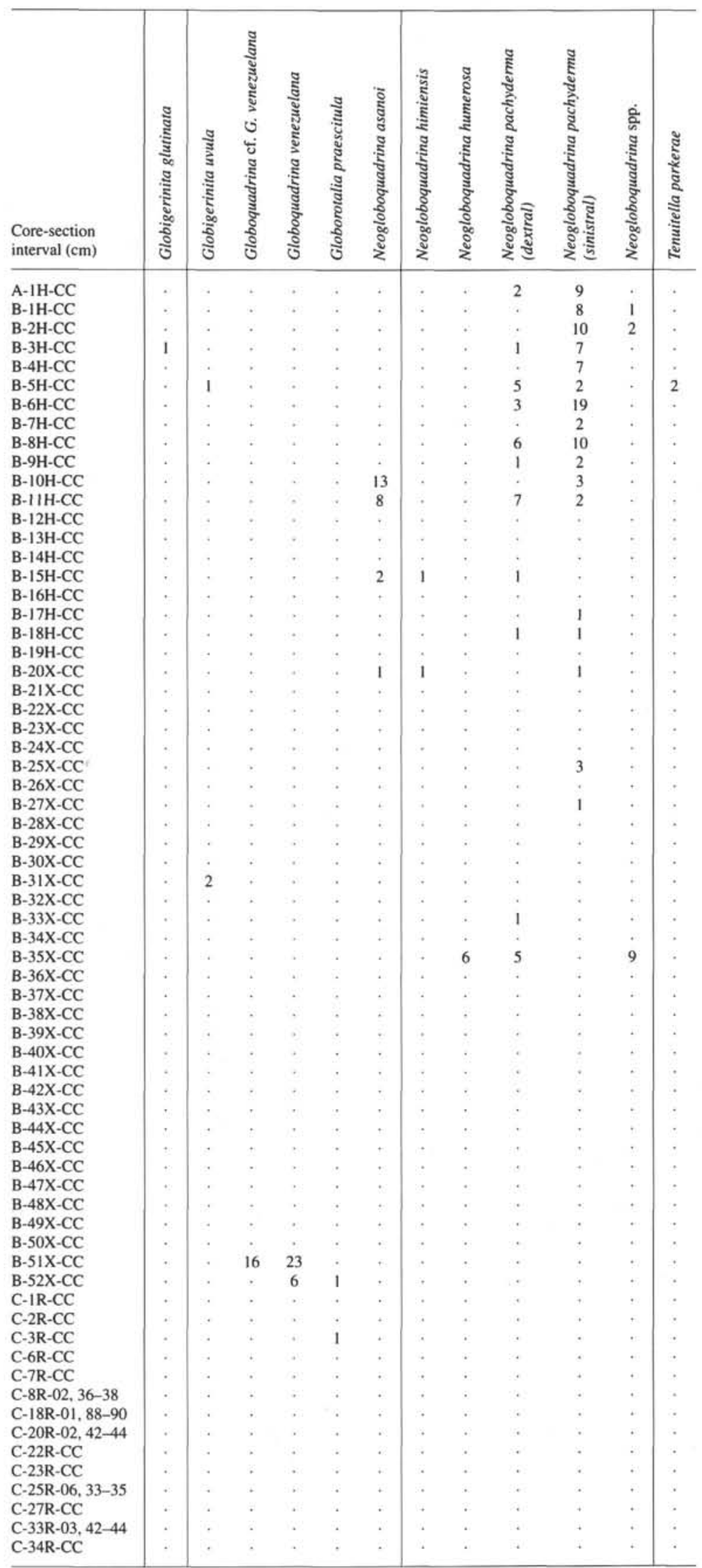


Table 6. Summary of benthic foraminifer upper depth limits and species distribution in the Yamato and Japan Basins.

\begin{tabular}{|c|c|c|c|c|c|c|c|c|c|c|}
\hline \multirow[b]{2}{*}{$\begin{array}{l}\text { Upper } \\
\text { Depth } \\
\text { Limit }\end{array}$} & \multirow[b]{2}{*}{ Species } & \multicolumn{5}{|c|}{ Yamato Basin } & \multicolumn{4}{|c|}{ Japan Basin } \\
\hline & & Quaternary & Pliocene & $\begin{array}{c}\text { upper } \\
\text { upper } \\
\text { Miocene }\end{array}$ & $\begin{array}{c}\text { lower } \\
\text { upper } \\
\text { Miocene }\end{array}$ & $\begin{array}{c}\text { mid- } \\
\text { lower } \\
\text { Miocene }\end{array}$ & Quaternary & Pliocene & $\begin{array}{c}\text { lower } \\
\text { upper } \\
\text { Miocene }\end{array}$ & $\begin{array}{c}\text { mid } \\
\text { Miocene }\end{array}$ \\
\hline $\mathrm{N}$ & Cibicides lobatulus & & & $\mathrm{X}$ & & & & & & \\
\hline $\mathrm{N}$ & Elphidium subincertum & & & $\mathrm{x}$ & & & & & & $\mathrm{X}$ \\
\hline $\mathrm{N}$ & Nonionella miocenica & & & & & $\mathrm{X}$ & & & $\mathrm{x}$ & $\mathrm{X}$ \\
\hline UB & Epistominella pacifica & $\mathrm{x}$ & & & & & $\mathrm{x}$ & & & \\
\hline$<$ UMB & Chilostomella oolina & & & & & $\mathrm{X}$ & & & $\mathrm{x}$ & \\
\hline$<$ UMB & Stainforthia complanata & $\mathrm{X}$ & & & & & $\mathrm{X}$ & & & \\
\hline$>$ UMB & Gyroidina orbicularis & & & & & $\mathrm{X}$ & & & & $\mathrm{X}$ \\
\hline UMB & Bulimina striata & $\mathrm{x}$ & & & & & $\mathrm{X}$ & & & \\
\hline$>\mathrm{UMB}$ & Cibicides wuellerstorfi & & & & & $\mathrm{X}$ & & & $\mathrm{X}$ & $\mathrm{X}$ \\
\hline$>\mathrm{UMB}$ & Oridorsalis tener & & & & & $\mathrm{X}$ & & & & \\
\hline$<\mathrm{MB}$ & Pullenia salisburyi & & & & $\mathrm{X}$ & & & & & \\
\hline $\mathrm{MB}$ & Islandiella noncrossi & $\mathrm{X}$ & & & & & $\mathrm{x}$ & & & \\
\hline LMB & Eggerella bradyi & & $\mathrm{X}$ & & & & & $\mathrm{X}$ & & \\
\hline LMB & $\begin{array}{c}\text { Globobulimina } \\
\text { auriculata }\end{array}$ & & $\mathrm{x}$ & $\mathrm{X}$ & $\mathrm{x}$ & $\mathrm{x}$ & & $\mathrm{x}$ & $\mathrm{x}$ & $\mathrm{x}$ \\
\hline$>\mathrm{LMB}$ & Martinortiella communis & & $\mathrm{x}$ & & & & & $\mathrm{X}$ & & \\
\hline$>\mathrm{LMB}$ & Pullenia bulloides & & & & & $\mathrm{X}$ & & & & $\mathrm{x}$ \\
\hline$>\mathrm{LMB}$ & Melonis pompilioides & & $\mathrm{x}$. & & & & & $\mathrm{X}$ & $\mathrm{X}$ & $\mathrm{x}$ \\
\hline$>\mathrm{LMB}$ & Gyroidina soldanii & & & & & & & & $\mathrm{X}$ & \\
\hline $\mathrm{N}$ & neritic depth $(<200 \mathrm{~m})$ & & & & & & & & & \\
\hline UB & upper bathyal depth (150- & $0 \mathrm{~m})$ & & & & & & & & \\
\hline$<\mathrm{UMB}$ & upper middle bathyal to sh & ower depths ( & $1,500 \mathrm{~m})$ & & & & & & & \\
\hline UMB & upper middle bathyal dept & $500-1,500 \mathrm{~m})$ & & & & & & & & \\
\hline$>$ UMB & upper middle bathyal to gr & ter depths $(>50$ & $0 \mathrm{~m})$ & & & & & & & \\
\hline$<\mathrm{MB}$ & middle bathyal to shallowe & lepths $(<2,500$ & & & & & & & & \\
\hline $\mathrm{MB}$ & middle bathyal depth (500 & $500 \mathrm{~m})$ & & & & & & & & \\
\hline LMB & lower middle bathyal dept & $1,500-2,500 \mathrm{n}$ & & & & & & & & \\
\hline$>\mathrm{LMB}$ & lower middle bathyal to gr & er depths $(>1$, & $00 \mathrm{~m})$ & & & & & & & \\
\hline
\end{tabular}

in the Japan Basin and the lower, upper, and middle upper Miocene in the Yamato Basin. Based on this evidence, low-oxygen bottom waters persisted through much of the Miocene but oxygenated bottom waters were well established by the uppermost Miocene, and similar conditions persisted throughout the Pliocene sequence. It is interesting to consider whether or not high-latitude Late Miocene cooling might have driven renewal of Japan Sea deep water.

\section{Event: Appearance of Anomalously Shallow Quaternary Fauna}

The Quaternary fauna appears shallower than that of the older sequences (Table 6) because it bears middle to upper bathyal species, but the present lower bathyal depth of the basin argues against such a shallowing. The anomalous fauna is either entirely displaced from shallow depth, or unusual conditions in the silled Japan Sea have excluded a lower bathyal fauna. The change to this unusual Quaternary fauna that was originally pointed out by Matoba (1984, and references therein) occurs near the Pliocene/Pleistocene Boundary rather than in the upper Pleistocene. The event may be related to shallowing of sills, which exclude from the marginal sea the deep fauna on the Pacific side of the Japan islands, and to altered bathyal conditions that exterminated the Pliocene fauna.

\section{Detailed Pliocene and Quaternary Planktonic Foraminifer Biostratigraphy}

Correlation of the Japan Sea sequences to the assemblage zones of Maiya et al. (1976) is not possible because abundant occurrences of planktonic foraminifers are limited mostly to the upper Quaternary. Furthermore, preservation is sporadic with many gaps within the upper Quaternary sequence. The deepest sample that bears planktonic foraminifers at Site 797 contains only Neogloboquadrina pachyderma with no Neogloboquadrina asanoi and lies within the uppermost Olduvai Subchron. This is consistent with the date of the last appearance of Neogloboquadrina asanoi at the base of the Olduvai Subchron as reported by Maiya et al. (1976) and confirmed by Lagoe and Thompson (1988).

Correlation to the coiling direction zones of Lagoe and Thompson (1988) is not possible because all Quaternary samples are dominated ( $>80 \%$ ) by sinistral Neogloboquadrina pachyderma (Table 10), and no dextral coiling intervals were found even though both sequences contain the Brunhes/Matuyama Boundary (at 38.4 mbsf in Hole 797B and at 24.6 or $34.1 \mathrm{mbsf}$ in Hole 794A; L. Vigliotti and J. Wippern, pers. comm., 1990) at which the CD8/CD9 coiling change boundary is expected from near $90 \%$ sinistral forms above to near $50 \%$ sinistral forms below. The ratio of $63 \%$ sinistral forms in the Olduvai sample of Site 797 is higher than predicted if it lies with Zone CD11, which spans the middle and upper Olduvai sequence. The Holocene interval of dextral coiling is absent either due to disturbance during the drill-string entry into the bottom or due to dissolution beneath the $\mathrm{CCD}$, which lies between 1,500 and 2,000 m water depth (Ichikura and Ujiié, 1976) in the Holocene Japan Sea.

\section{Character of the Upper Water Column during the Quaternary}

Cluster analysis separates four distinct groupings of planktonic foraminifers in this data set (Fig. 3, Table 11). Cluster 1 is a cold subarctic fauna produced during isothermal subarctic conditions, Cluster 2 is a well-preserved subarctic fauna produced by seasonal alternation of isothermal and stratified conditions, Cluster 3 is a subarctic fauna that appears similar to the more-dissolved end member of Cluster 2, and Cluster 4 is an upwelling(?) fauna.

The succession of clusters up-hole can be interpreted environmentally (Fig. 4). The deepest sample at Hole 797B is an unclustered sample with an assemblage most like that of the present-day transitional biogeographic province (Coulbourn et al., 1980). Between 50 and $40 \mathrm{mbsf}$, samples alternate between the cold, subpolar Cluster 1 and the seasonal, well-preserved subpolar Cluster 2. Near the 
Table 7. Cores bearing a low oxygen facies determined by style of bioturbation and by foraminifer assemblage.

\begin{tabular}{|c|c|c|c|}
\hline \multicolumn{2}{|c|}{ Yamato Basin } & \multicolumn{2}{|c|}{ Japan Basin } \\
\hline $\begin{array}{l}\text { Occurrences of horizontally } \\
\text { flattened burrows and } \\
\text { laminations }\end{array}$ & $\begin{array}{c}\text { Occurrences of Cyclammina- } \\
\text { Haplophragmoides- } \\
\text { Ammodiscus assemblage }\end{array}$ & $\begin{array}{l}\text { Occurrences of horizontally } \\
\text { flattened burrows and } \\
\text { laminations }\end{array}$ & $\begin{array}{c}\text { Occurrences of Cyclammina- } \\
\text { Haplophragmoides- } \\
\text { Ammodiscus assemblage }\end{array}$ \\
\hline Hole 794B & Hole 794B & Hole 796B & Hole 796B \\
\hline $12 \mathrm{R}$ & & $11 \mathrm{R}$ & \\
\hline $13 R$ & $13 R$ & $12 \mathrm{R}$ & \\
\hline $14 \mathrm{R}$ & $14 \mathrm{R}$ & $14 \mathrm{R}$ & \\
\hline $15 \mathrm{R}$ & & $15 \mathrm{R}$ & \\
\hline $16 \mathrm{R}$ & & $18 \mathrm{R}$ & \\
\hline $17 \mathrm{R}$ & & & $19 \mathrm{R}$ \\
\hline & $18 \mathrm{R}$ & $28 \mathrm{R}$ & $28 \mathrm{R}$ \\
\hline $20 \mathrm{R}$ & & $29 \mathrm{R}$ & $29 \mathrm{R}$ \\
\hline $21 \mathrm{R}$ & & $30 \mathrm{R}$ & \\
\hline $22 \mathrm{R}$ & & & $31 \mathrm{R}$ \\
\hline $24 \mathrm{R}$ & $24 \mathrm{R}$ & $32 \mathrm{R}$ & $32 \mathrm{R}$ \\
\hline $25 \mathrm{R}$ & $25 \mathrm{R}$ & & \\
\hline $26 \mathrm{R}$ & $26 \mathrm{R}$ & & \\
\hline \multirow[t]{2}{*}{ Hole 797C } & Hole 797C & Hole 795B & Hole 795B \\
\hline & $33 x$ & IR & \\
\hline \multirow[t]{4}{*}{$34 X$} & & $2 \mathrm{R}$ & \\
\hline & $35 \mathrm{X}$ & $3 \mathrm{R}$ & \\
\hline & $36 \mathrm{X}$ & $7 R$ & $7 \mathrm{R}$ \\
\hline & $37 X$ & 10R & 10R \\
\hline $41 X$ & & $11 \mathrm{R}$ & $11 \mathrm{R}$ \\
\hline $43 X$ & & $12 \mathrm{R}$ & $12 \mathrm{R}$ \\
\hline $44 X$ & & $13 R$ & $13 \mathrm{R}$ \\
\hline $46 \mathrm{X}$ & & $14 \mathrm{R}$ & $14 \mathrm{R}$ \\
\hline $47 X$ & $47 X$ & $15 \mathrm{R}$ & $15 \mathrm{R}$ \\
\hline $48 \mathrm{X}$ & $48 \mathrm{X}$ & $16 \mathrm{R}$ & $16 \mathrm{R}$ \\
\hline $49 \mathrm{X}$ & & $17 \mathrm{R}$ & $17 \mathrm{R}$ \\
\hline $51 X$ & $51 X$ & $18 \mathrm{R}$ & $18 \mathrm{R}$ \\
\hline $52 \mathrm{X}$ & $52 X$ & $19 \mathrm{R}$ & \\
\hline \multirow[t]{2}{*}{$2 \mathrm{R}$} & $2 \mathrm{R}$ & & $20 \mathrm{R}$ \\
\hline & $3 R$ & $21 \mathrm{R}$ & $21 \mathrm{R}$ \\
\hline $5 R$ & & $22 \mathrm{R}$ & \\
\hline \multirow[t]{2}{*}{$6 \mathrm{R}$} & $6 \mathrm{R}$ & $23 \mathrm{R}$ & $23 \mathrm{R}$ \\
\hline & $7 R$ & $24 \mathrm{R}$ & $24 \mathrm{R}$ \\
\hline \multirow[t]{8}{*}{$8 R$} & $8 \mathrm{R}$ & $25 \mathrm{R}$ & \\
\hline & & $26 \mathrm{R}$ & \\
\hline & & $27 R$ & \\
\hline & & $28 \mathrm{R}$ & $28 \mathrm{R}$ \\
\hline & & $29 \mathrm{R}$ & $29 \mathrm{R}$ \\
\hline & & $30 \mathrm{R}$ & $30 \mathrm{R}$ \\
\hline & & $31 \mathrm{R}$ & \\
\hline & & $32 \mathrm{R}$ & \\
\hline
\end{tabular}

Brunhes/Matuyama boundary at $38.40 \mathrm{mbsf}$, samples alternate between the cold subpolar Cluster 1 and the seasonal, somewhat dissolved Cluster 3 . Between 35 and 28 mbsf, approximately 0.57 $\mathrm{Ma}$, the surface-water column alternates between the seasonal subarctic conditions represented by Cluster 2 and upwelling(?) conditions represented by Cluster 4 . Above $25 \mathrm{mbsf}$, conditions return to those seen deeper in the core (between 50 and $40 \mathrm{mbsf}$ ) and alternate between the cold, subpolar Cluster 1 and the seasonal, well-preserved subpolar Cluster 2. A similar alternation of these two clusters is observed in the upper Pleistocene sequence of Hole 794A.

The four assemblages defined by the cluster analysis resemble the assemblages in latest Pleistocene piston cores from the Japan Sea, including the Globigerina bulloides enriched assemblage of Cluster 4 (Ujiié and Ichikura, 1973; Ichikura and Ujiié, 1976; Kato, 1984). In contrast, the Olduvai sample of Hole 797B has no latest Pleistocene or Holocene analog in the Japan Sea, but resembles the fauna of the modern transitional North Pacific Ocean (Coulbourn et al., 1980).

\section{CONCLUSIONS}

1. The abundance of foraminifers, in general, is low at all sites in the deep Japan and Yamato basins. Preservation, diversity, and abundance of the calcareous fauna are best at Site 797 and worst at Site 794. The assemblages of agglutinated foraminifers are best developed at Sites 795 and 796 in the Japan Basin.

2. The succession of benthic foraminifers in the Neogene sequences can be assigned to the zonation of Matsunaga (1963), whereas the planktonic foraminifers of the Quaternary cannot be assigned to the zonations of Maiya et al. (1976) or Lagoe and Thompson (1988).

3. Both the Japan and Yamato basins had subsided to lower middle bathyal depths by the time the first foraminifer-bearing sediments were deposited above igneous basement at the three sites where basement was drilled.

4. The Miocene cooling of the Japan Basin occurred in the early middle Miocene. The data set does not yield improved resolution of the event compared to land-based sequences. 
Table 8. Listing of data including total sediment weight, weight of sand-size fraction, percent sand-size material by weight, fraction (split) of sample examined for foraminifers, planktonic and benthic foraminifer numbers, benthic/planktonic ratio, coiling ratio of Neogloboquadrina pachyderma, the number of planktonic and benthic tests counted, and relative frequency taxa of planktonic foraminifers.

\begin{tabular}{|c|c|c|c|c|c|c|c|c|c|c|c|c|c|c|c|c|c|c|c|c|c|c|}
\hline Sample 127 . & $\begin{array}{l}\text { Depth } \\
\text { (mbsf) }\end{array}$ & $\begin{array}{l}\text { Sand } \\
\text { Weight }\end{array}$ & $\begin{array}{c}\text { Sed } \\
\text { Weight }\end{array}$ & $\begin{array}{c}\text { Sand } \\
\tilde{\%}\end{array}$ & Split & $\begin{array}{c}\text { Pla } \\
\text { Num }\end{array}$ & $\begin{array}{l}\text { Ben } \\
\text { Num }\end{array}$ & $\begin{array}{l}\text { B/P } \\
\text { Rat }\end{array}$ & $\begin{array}{l}\text { Coil } \\
\text { Rat }\end{array}$ & $\begin{array}{c}\# \\
\mathrm{Pla}\end{array}$ & $\begin{array}{c}\# \\
\text { Ben }\end{array}$ & $\begin{array}{c}\text { PacL } \\
\%\end{array}$ & $\begin{array}{c}\text { PacR } \\
\%\end{array}$ & $\begin{array}{l}\text { Bul } \\
\%\end{array}$ & $\underset{\%}{\text { Umb }}$ & $\underset{\%}{\text { Qui }}$ & $\underset{\%}{\text { Glu }}$ & $\underset{\%}{\operatorname{Min}}$ & $\begin{array}{l}\text { Uvu } \\
\%\end{array}$ & $\begin{array}{l}\text { Par } \\
\%\end{array}$ & $\underset{\%}{\mathrm{Hum}}$ & $\begin{array}{l}\text { Ind } \\
\text { \% }\end{array}$ \\
\hline $794 \mathrm{~A}-1 \mathrm{H}-1,2-4 \mathrm{~cm}$ & 0.02 & 0.1183 & 6.8940 & 1.72 & 1.0000 & 0 & 0 & 0 & 0 & 0 & 0 & 0 & $\mathbf{0}$ & 0 & 0 & 0 & 0 & 0 & 0 & 0 & 0 & 0 \\
\hline $794 \mathrm{~A}-1 \mathrm{H}-1.62-64$ & 0.62 & 0.0107 & 4.5130 & 0.24 & 1.0000 & 0 & 0 & 0 & 0 & 0 & 0 & 0 & 0 & 0 & 0 & 0 & 0 & 0 & 0 & 0 & 0 & 0 \\
\hline 794A-1H-1, 93-98 & 0.93 & 0.0431 & 13.2410 & 0.33 & 1.0000 & 3 & 0 & 0 & 100 & 46 & 0 & 24 & 0 & 74 & 0 & 2 & 0 & 0 & 0 & 0 & 0 & 0 \\
\hline $794 \mathrm{~A}-1 \mathrm{H}-1.135-137$ & 1.35 & 0.1957 & 20.4730 & 0.96 & 1.0000 & 0 & 0 & $\mathbf{0}$ & 0 & 0 & 0 & 0 & 0 & 0 & 0 & 0 & 0 & 0 & 0 & 0 & 0 & 0 \\
\hline $794 \mathrm{~A}-1 \mathrm{H}-2,21-24$ & 1.71 & 0.2101 & 10.2760 & 2.04 & 1.0000 & 0 & 0 & 0 & 0 & 3 & 0 & 0 & 0 & 100 & 0 & 0 & 0 & 0 & 0 & 0 & 0 & 0 \\
\hline $794 \mathrm{~A}-1 \mathrm{H}-2,65-67$ & 2.15 & 0.0852 & 5.6200 & 1.52 & 1.0000 & 0 & 0 & 0 & 100 & 1 & 0 & 100 & 0 & 0 & 0 & 0 & 0 & 0 & 0 & 0 & 0 & 0 \\
\hline $794 \mathrm{~A}-1 \mathrm{H}-2,93-98$ & 2.43 & 0.3309 & 13.2630 & 2.49 & 1.0000 & 0 & 0 & 0 & 0 & 0 & 0 & 0 & 0 & 0 & 0 & 0 & 0 & 0 & 0 & 0 & 0 & 0 \\
\hline $794 \mathrm{~A}-1 \mathrm{H}-2,133-136$ & 2.83 & 0.1333 & 8.8800 & 1.50 & 1.0000 & 0 & 0 & 0 & 0 & 0 & 0 & 0 & 0 & 0 & 0 & 0 & 0 & 0 & 0 & 0 & 0 & 0 \\
\hline $794 \mathrm{~A}-1 \mathrm{H}-3,26-28$ & 3.26 & 0.3270 & 10.8710 & 3.01 & 1.0000 & 0 & 0 & 0 & 0 & 0 & 0 & 0 & 0 & 0 & 0 & $\mathbf{0}$ & 0 & 0 & 0 & 0 & 0 & 0 \\
\hline $794 \mathrm{~A}-1 \mathrm{H}-3,65-67$ & 3.65 & 0.0105 & 5.2680 & 0.20 & 1.0000 & 0 & 0 & 0 & 0 & 1 & 0 & 0 & 0 & 0 & 0 & 0 & 100 & 0 & 0 & 0 & 0 & 0 \\
\hline $794 \mathrm{~A}-1 \mathrm{H}-3,69-70$ & 3.69 & 0.0253 & 2.3920 & 1.06 & 1.0000 & 1 & 0 & 0 & 100 & 2 & 0 & so & 0 & 0 & 0 & 50 & 0 & 0 & 0 & 0 & 0 & 0 \\
\hline $794 \mathrm{~A}-1 \mathrm{H}-3,93-98$ & 3.93 & 0.1156 & 11.4460 & 1.01 & 1.0000 & 4 & 0 & 8 & 100 & 48 & 4 & 85 & $\mathbf{0}$ & 12 & 0 & 2 & 0 & 0 & 0 & 0 & 0 & 0 \\
\hline $794 \mathrm{~A}-1 \mathrm{H}-4,26-31$ & 4.76 & 0.0275 & 23.0630 & 0.12 & 1.0000 & 0 & 0 & 0 & 0 & 0 & 0 & 0 & 0 & 0 & 0 & 0 & 0 & 0 & 0 & 0 & 0 & 0 \\
\hline $794 \mathrm{~A}-1 \mathrm{H}-4,56-58$ & 5.06 & 0.0104 & 7.3590 & 0.14 & 1.0000 & 0 & 0 & 100 & 0 & 0 & 1 & 0 & $\mathbf{0}$ & 0 & 0 & $\mathbf{0}$ & 0 & 0 & 0 & 0 & 0 & 0 \\
\hline $794 \mathrm{~A}-1 \mathrm{H}-4,92-94$ & 5.42 & 0.0093 & 6.9860 & 0.13 & 1.0000 & 0 & 0 & 0 & 0 & 0 & 0 & 0 & 0 & 0 & 0 & 0 & 0 & 0 & 0 & 0 & $\mathbf{0}$ & 0 \\
\hline $794 \mathrm{~A}-1 \mathrm{H}-4,136-138$ & 5.86 & 0.0192 & 7.2040 & 0.27 & 1.0000 & 0 & 0 & 0 & 0 & 0 & 0 & 0 & 0 & 0 & 0 & 0 & 0 & 0 & 0 & 0 & $\mathbf{0}$ & 0 \\
\hline $794 \mathrm{~A}-1 \mathrm{H}-5.3-5$ & 6.03 & 0.0000 & 11.5100 & 0.00 & 1.0000 & 0 & 0 & 0 & 0 & 0 & 0 & 0 & 0 & 0 & 0 & 0 & 0 & 0 & 0 & 0 & 0 & 0 \\
\hline $794 \mathrm{~A}-1 \mathrm{H}-5,25-27$ & 6.25 & 0.6584 & 11.1990 & 5.88 & 1.0000 & 0 & 0 & 0 & 0 & 0 & 0 & 0 & 0 & 0 & 0 & 0 & 0 & 0 & 0 & 0 & 0 & 0 \\
\hline $794 \mathrm{~A}-2 \mathrm{H}-1,22-24$ & 7.02 & 0.0220 & 10.9710 & 0.20 & 1.0000 & $\mathbf{0}$ & 0 & 0 & 0 & 0 & 0 & 0 & 0 & 0 & 0 & $\mathbf{0}$ & 0 & 0 & 0 & $\mathbf{0}$ & 0 & 0 \\
\hline $794 \mathrm{~A}-2 \mathrm{H}-1,50-53$ & 7.30 & 0.0131 & 8.1950 & 0.16 & 1.0000 & 0 & 0 & 0 & $\mathbf{0}$ & 0 & 0 & 0 & 0 & 0 & 0 & 0 & 0 & 0 & 0 & 0 & 0 & 0 \\
\hline $794 \mathrm{~A}-2 \mathrm{H}-1,96-98$ & 7.76 & 0.1209 & 9.8870 & 1.22 & 1.0000 & 0 & 0 & 0 & $\mathbf{0}$ & 0 & 0 & 0 & 0 & 0 & 0 & 0 & 0 & 0 & 0 & 0 & 0 & 0 \\
\hline $794 \mathrm{~A}-2 \mathrm{H}-1.118-120$ & 7.98 & 0.0648 & 10.8450 & 0.60 & 1.0000 & 0 & 0 & 0 & 0 & 0 & 0 & 0 & $\mathbf{0}$ & 0 & 0 & 0 & 0 & $\mathbf{0}$ & 0 & 0 & 0 & 0 \\
\hline $794 \mathrm{~A}-2 \mathrm{H}-1,142-144$ & 8.22 & 0.1935 & 11.4960 & 1.68 & 1.0000 & 0 & 0 & 0 & 0 & 0 & 0 & 0 & 0 & 0 & 0 & 0 & 0 & 0 & 0 & 0 & 0 & 0 \\
\hline $794 \mathrm{~A}-2 \mathrm{H}-2,10-12$ & 8.40 & 0.2570 & 12.8400 & 2.00 & 1.0000 & 0 & 0 & 0 & 0 & 0 & 0 & 0 & 0 & 0 & 0 & $\mathbf{0}$ & 0 & $\mathbf{0}$ & 0 & 0 & $\mathbf{0}$ & 0 \\
\hline $794 \mathrm{~A}-2 \mathrm{H}-2,50-52$ & 8.80 & 0.2041 & 12.3210 & 1.66 & 1.0000 & 0 & 0 & 0 & 0 & 0 & 0 & 0 & 0 & 0 & 0 & 0 & 0 & 0 & 0 & 0 & 0 & 0 \\
\hline $794 \mathrm{~A}-2 \mathrm{H}-2,113-116$ & 9.43 & 0.0973 & 9.6230 & 1.01 & 1.0000 & 0 & 0 & 0 & 0 & 0 & 0 & 0 & 0 & 0 & 0 & 0 & 0 & 0 & 0 & 0 & 0 & 0 \\
\hline $794 \mathrm{~A}-2 \mathrm{H}-2,136-138$ & 9.66 & 0.0936 & 11.7460 & 0.80 & 1.0000 & 0 & 0 & 0 & 0 & 0 & 0 & 0 & 0 & 0 & 0 & 0 & 0 & 0 & 0 & 0 & 0 & 0 \\
\hline $794 \mathrm{~A}-2 \mathrm{H}-3,8-11$ & 9.88 & 0.0505 & 10.8840 & 0.46 & 1.0000 & 0 & 0 & 0 & 0 & 0 & 0 & 0 & 0 & 0 & 0 & 0 & 0 & 0 & 0 & 0 & 0 & 0 \\
\hline $794 \mathrm{~A}-2 \mathrm{H}-3,56-58$ & 10.36 & 0.0166 & 8.9580 & 0.19 & 1.0000 & 0 & 0 & 0 & $\mathbf{0}$ & 0 & 0 & 0 & 0 & $\mathbf{0}$ & 0 & $\mathbf{0}$ & 0 & 0 & 0 & 0 & $\mathbf{0}$ & 0 \\
\hline $794 \mathrm{~A}-2 \mathrm{H}-3,96-98$ & 10.76 & 0.0019 & 9.8430 & 0.02 & 1.0000 & 0 & 0 & 0 & 0 & 0 & 0 & 0 & 0 & 0 & 0 & 0 & 0 & 0 & 0 & 0 & 0 & 0 \\
\hline $794 \mathrm{~A}-2 \mathrm{H}-3,127-129$ & 11.07 & 0.1009 & 12.4930 & 0.81 & 1.0000 & 0 & 0 & 0 & 0 & 0 & 0 & 0 & $\mathbf{0}$ & 0 & 0 & 0 & 0 & 0 & $\mathbf{0}$ & 0 & $\mathbf{0}$ & $\mathbf{0}$ \\
\hline $794 \mathrm{~A}-2 \mathrm{H}-3,135-138$ & 11.15 & 0.1010 & 9.3420 & 1.08 & 1.0000 & 0 & 0 & 0 & 0 & 0 & 0 & 0 & 0 & 0 & 0 & 0 & 0 & 0 & $\mathbf{0}$ & 0 & 0 & $\mathbf{0}$ \\
\hline $794 \mathrm{~A}-2 \mathrm{H}-4,17-20$ & 11.47 & 0.1638 & 12.1510 & 1.35 & 1.0000 & 0 & 0 & 0 & 0 & 0 & 0 & 0 & 0 & 0 & 0 & 0 & 0 & 0 & 0 & 0 & 0 & 0 \\
\hline $794 \mathrm{~A}-2 \mathrm{H}-4,46-49$ & 11.76 & 0.0883 & 11.8170 & 0.75 & 1.0000 & 0 & 0 & 0 & 0 & 0 & 0 & 0 & $\mathbf{0}$ & 0 & 0 & 0 & 0 & 0 & 0 & 0 & 0 & 0 \\
\hline $794 \mathrm{~A}-2 \mathrm{H}-4,87-89$ & 12.17 & 0.0062 & 7.9228 & 0.08 & 1.0000 & 0 & 0 & 0 & 0 & 0 & 0 & 0 & 0 & 0 & 0 & 0 & 0 & 0 & 0 & 0 & 0 & 0 \\
\hline $794 \mathrm{~A}-2 \mathrm{H}-4,96-98$ & 12.26 & 0.0000 & 7.3570 & 0.00 & 1.0000 & 0 & 0 & $\mathbf{0}$ & $\mathbf{0}$ & 0 & 0 & 0 & 0 & 0 & 0 & 0 & 0 & 0 & 0 & 0 & 0 & 0 \\
\hline $794 \mathrm{~A}-2 \mathrm{H}-4,132-135$ & 12.62 & 0.0039 & 9.3340 & 0.04 & 1.0000 & 0 & 0 & 0 & $\mathbf{0}$ & 0 & 0 & 0 & 0 & $\mathbf{0}$ & 0 & 0 & 0 & 0 & $\mathbf{0}$ & 0 & 0 & 0 \\
\hline $794 \mathrm{~A}-2 \mathrm{H}-5,10-12$ & 12.90 & 0.0052 & 8.2900 & 0.06 & 1.0000 & 0 & 0 & 0 & $\mathbf{0}$ & 0 & 0 & 0 & 0 & 0 & 0 & 0 & 0 & $\mathbf{0}$ & 0 & 0 & 0 & 0 \\
\hline $794 \mathrm{~A}-2 \mathrm{H}-5,39-42$ & 13,19 & 0.0191 & 7.1140 & 0.27 & 1.0000 & 0 & 0 & 0 & 0 & 0 & 0 & 0 & 0 & 0 & 0 & 0 & 0 & 0 & 0 & 0 & 0 & 0 \\
\hline $794 \mathrm{~A}-2 \mathrm{H}-5,77-79$ & 13.57 & 0.0398 & 9.2140 & 0.43 & 1.0000 & 0 & 0 & $\mathbf{0}$ & 0 & 0 & 0 & 0 & 0 & $\mathbf{0}$ & 0 & 0 & 0 & 0 & $\mathbf{0}$ & 0 & 0 & 0 \\
\hline $794 \mathrm{~A}-2 \mathrm{H}-5,96-98$ & 13.76 & 0.1380 & 8.3130 & 1.66 & 1.0000 & 0 & 0 & 0 & $\mathbf{0}$ & 0 & 0 & 0 & 0 & 0 & 0 & 0 & 0 & 0 & 0 & 0 & 0 & 0 \\
\hline $794 \mathrm{~A}-2 \mathrm{H}-5,126-128$ & 14.06 & 0.1262 & 10.4854 & 1.20 & 1.0000 & 0 & 0 & 0 & 0 & 0 & 0 & 0 & 0 & 0 & 0 & 0 & 0 & 0 & 0 & 0 & $\mathbf{0}$ & 0 \\
\hline $794 \mathrm{~A}-2 \mathrm{H}-6.8-10$ & 14.38 & 0.1267 & 8.1140 & 1.56 & 1.0000 & 0 & 0 & 0 & 0 & 0 & 0 & 0 & $\mathbf{0}$ & $\mathbf{0}$ & 0 & $\mathbf{0}$ & 0 & 0 & 0 & 0 & 0 & 0 \\
\hline $794 \mathrm{~A}-2 \mathrm{H}-6,17-20$ & 14.47 & 0.3739 & 12.0690 & 3.10 & 0.0039 & 6416 & 191 & 3 & 98 & 302 & 9 & 92 & 2 & 6 & 0 & 0 & 0 & 0 & $\mathbf{0}$ & 0 & $\mathbf{0}$ & 0 \\
\hline $794 \mathrm{~A}-2 \mathrm{H}-6,84-87$ & 15.14 & 0.0608 & 10.6740 & 0.57 & 1.0000 & 42 & 0 & 0 & 94 & 444 & 0 & 38 & 2 & 58 & 1 & 1 & 0 & 0 & 0 & 0 & 0 & $\mathbf{0}$ \\
\hline $794 \mathrm{~A}-2 \mathrm{H}-6,91-93$ & 15.21 & 0.2146 & 7.1680 & 2.99 & 1.0000 & 0 & 0 & 0 & 0 & 0 & 0 & 0 & 0 & 0 & 0 & 0 & 0 & 0 & 0 & 0 & 0 & 0 \\
\hline $794 \mathrm{~A}-2 \mathrm{H}-6,96-98$ & 15.26 & 0.8340 & 10.9830 & 7.59 & 0.0010 & 27770 & 1457 & 5 & 92 & 305 & 16 & 51 & 4 & 13 & 0 & 6 & 0 & 0 & 1 & 10 & 13 & 1 \\
\hline $794 \mathrm{~A}-2 \mathrm{H}-6,99-102$ & 15.29 & 0.0213 & 6.8260 & 0.31 & 1.0000 & 1 & 0 & 0 & 100 & 5 & 0 & 40 & 0 & 60 & 0 & 0 & 0 & 0 & 0 & 0 & 0 & 0 \\
\hline $794 \mathrm{~A}-2 \mathrm{H}-7,8-10$ & 15.88 & 0.0164 & 8.4150 & 0.19 & 1.0000 & 0 & 0 & 0 & 0 & 0 & 0 & 0 & 0 & 0 & 0 & 0 & 0 & 0 & 0 & 0 & 0 & 0 \\
\hline $794 \mathrm{~A}-2 \mathrm{H}-7,48-50$ & 16.28 & 0.0077 & 6.6374 & 0.12 & 1.0000 & 0 & 0 & 0 & 0 & 0 & 0 & 0 & 0 & 0 & 0 & 0 & 0 & 0 & $\mathbf{0}$ & 0 & 0 & 0 \\
\hline $794 \mathrm{~A}-3 \mathrm{H}-1,23-25$ & 16.53 & 0.0252 & 7.6680 & 0.33 & 1.0000 & 0 & 0 & 0 & 0 & 0 & 0 & 0 & 0 & 0 & 0 & 0 & 0 & 0 & $\mathbf{0}$ & 0 & 0 & 0 \\
\hline $794 \mathrm{~A}-3 \mathrm{H}-1,65-67$ & 16.95 & 0.1296 & 9.7010 & 1.34 & 1.0000 & 0 & 0 & 0 & 0 & 0 & 0 & 0 & 0 & 0 & 0 & 0 & 0 & 0 & $\mathbf{0}$ & 0 & 0 & 0 \\
\hline $794 \mathrm{~A}-3 \mathrm{H}-1,94-99$ & 17.24 & 1.2430 & 21.1400 & 5.88 & 0.0012 & 13836 & 394 & 3 & 99 & 351 & 10 & 67 & 1 & 14 & 0 & 17 & 0 & 0 & 0 & 1 & 0 & 0 \\
\hline
\end{tabular}


Table 8 (continued).

\begin{tabular}{|c|c|c|c|c|c|c|c|c|c|c|c|c|c|c|c|c|c|c|c|c|c|c|}
\hline Sample 127. & $\begin{array}{l}\text { Depth } \\
\text { (mbsf) }\end{array}$ & $\begin{array}{l}\text { Sand } \\
\text { Weight }\end{array}$ & $\begin{array}{c}\text { Sed } \\
\text { Weight }\end{array}$ & $\begin{array}{l}\text { Sand } \\
\%\end{array}$ & Split & $\begin{array}{l}\mathrm{Pla} \\
\text { Num }\end{array}$ & $\begin{array}{l}\text { Ben } \\
\text { Num }\end{array}$ & $\begin{array}{l}\mathrm{B} / \mathrm{P} \\
\text { Rat }\end{array}$ & $\begin{array}{l}\text { Coil } \\
\text { Rat }\end{array}$ & $\stackrel{\#}{\mathrm{Pla}}$ & $\stackrel{\#}{\# \text { Ben }}$ & $\begin{array}{c}\text { PacL } \\
\%\end{array}$ & $\begin{array}{c}\text { PacR } \\
\%\end{array}$ & $\begin{array}{l}\text { Bul } \\
\%\end{array}$ & $\underset{\%}{\text { Umb }}$ & $\begin{array}{l}\text { Qui } \\
\%\end{array}$ & $\begin{array}{l}\text { Glu } \\
\%\end{array}$ & $\underset{\%}{\operatorname{Min}}$ & $\begin{array}{l}\text { Uvu } \\
\%\end{array}$ & $\begin{array}{l}\text { Par } \\
\%\end{array}$ & $\underset{\%}{\operatorname{Hum}}$ & $\begin{array}{l}\text { Ind } \\
\%\end{array}$ \\
\hline $794 \mathrm{~A}-3 \mathrm{H}-1,117-120$ & 17.47 & 1.0276 & 10.8870 & 9.44 & 0.0020 & 22366 & 1056 & 5 & 98 & 487 & 23 & 77 & 1 & 18 & $\mathbf{0}$ & 1 & 0 & 0 & 0 & 2 & 0 & 0 \\
\hline $794 \mathrm{~A}-3 \mathrm{H}-2,19-22$ & 17.99 & 0.1002 & 10.1460 & 0.99 & 1.0000 & 0 & 0 & 0 & 0 & 0 & 0 & 0 & 0 & 0 & 0 & 0 & $\mathbf{0}$ & 0 & 0 & 0 & 0 & 0 \\
\hline $794 \mathrm{~A}-3 \mathrm{H}-2,65-67$ & 18.45 & 0.1938 & 11.4190 & 1.70 & 1.0000 & 0 & 0 & 0 & 0 & 0 & 0 & 0 & 0 & 0 & 0 & 0 & $\mathbf{0}$ & 0 & 0 & $\mathbf{0}$ & 0 & 0 \\
\hline $794 \mathrm{~A}-3 \mathrm{H}-2,119-121$ & 18.99 & 0.0210 & 6.8491 & 0.31 & 1.0000 & 0 & 0 & 0 & 0 & 0 & 0 & 0 & 0 & 0 & 0 & 0 & 0 & 0 & 0 & $\mathbf{0}$ & 0 & 0 \\
\hline $794 \mathrm{~A}-3 \mathrm{H}-3,5-7$ & 19.35 & 0.0083 & 9.1821 & 0.09 & 1.0000 & 0 & 0 & 0 & 0 & 0 & 0 & 0 & 0 & 0 & 0 & 0 & 0 & 0 & 0 & 0 & 0 & 0 \\
\hline $794 \mathrm{~A}-3 \mathrm{H}-3,46-49$ & 19.76 & 0.0386 & 12.9780 & 0.30 & 1.0000 & 0 & 0 & 0 & 0 & 0 & 0 & 0 & 0 & 0 & 0 & 0 & 0 & 0 & 0 & 0 & 0 & 0 \\
\hline $794 \mathrm{~A}-3 \mathrm{H}-3,119-121$ & 20.49 & 0.2012 & 5.0730 & 3.97 & 1.0000 & 0 & 0 & $\mathbf{0}$ & 0 & 0 & 0 & 0 & 0 & 0 & 0 & 0 & 0 & 0 & 0 & 0 & 0 & 0 \\
\hline $794 \mathrm{~A}-3 \mathrm{H}-4,24-26$ & 21.04 & 0.3263 & 14.7640 & 2.21 & 1.0000 & 0 & 0 & 0 & 0 & 0 & 0 & 0 & 0 & 0 & 0 & 0 & 0 & 0 & 0 & 0 & 0 & 0 \\
\hline $794 \mathrm{~A}-3 \mathrm{H}-4,51-53$ & 21.31 & 0.7120 & 12.5220 & 5.69 & 1.0000 & 0 & 0 & 0 & 0 & 0 & 0 & 0 & $\mathbf{0}$ & 0 & 0 & 0 & 0 & 0 & 0 & $\mathbf{0}$ & 0 & 0 \\
\hline $794 \mathrm{~A}-3 \mathrm{H}-4,94-99$ & 21.74 & 0.0767 & 21.8600 & 0.35 & 1.0000 & 0 & 0 & 0 & 100 & 6 & 0 & 100 & 0 & 0 & 0 & 0 & 0 & 0 & 0 & 0 & 0 & 0 \\
\hline $794 \mathrm{~A}-3 \mathrm{H}-4,119-122$ & 21.99 & 0.0901 & 10.2760 & 0.88 & 1.0000 & 0 & 0 & 0 & 0 & 0 & 0 & 0 & 0 & 0 & 0 & 0 & 0 & 0 & 0 & 0 & 0 & 0 \\
\hline $794 \mathrm{~A}-3 \mathrm{H}-5,31-33$ & 22.61 & 0.0804 & 10.4860 & 0.77 & 1.0000 & 0 & 0 & 0 & 0 & 0 & 0 & 0 & 0 & 0 & 0 & 0 & 0 & 0 & 0 & 0 & 0 & 0 \\
\hline $794 \mathrm{~A}-3 \mathrm{H}-5,65-67$ & 22.95 & 0.0755 & 12.8380 & 0.59 & 1.0000 & 0 & 0 & 0 & 0 & 0 & 0 & 0 & 0 & 0 & 0 & 0 & 0 & 0 & 0 & 0 & 0 & 0 \\
\hline 794A-3H-5, 93-98 & 23.23 & 0.0841 & 20.5460 & 0.41 & 1.0000 & 0 & 0 & 0 & 0 & 0 & 0 & 0 & 0 & 0 & 0 & 0 & 0 & 0 & 0 & 0 & 0 & 0 \\
\hline $794 \mathrm{~A}-3 \mathrm{H}-5,116-118$ & 23.46 & 0.1282 & 13.0280 & 0.98 & 1.0000 & 0 & 0 & $\mathbf{0}$ & 0 & 0 & 0 & 0 & $\mathbf{0}$ & 0 & 0 & 0 & 0 & 0 & 0 & 0 & 0 & 0 \\
\hline $794 \mathrm{~A}-3 \mathrm{H}-6,25-27$ & 24.05 & 0.0080 & 9.6983 & 0.08 & 1.0000 & 0 & 0 & 0 & 0 & 0 & 0 & 0 & 0 & 0 & 0 & 0 & 0 & 0 & 0 & 0 & 0 & 0 \\
\hline $794 \mathrm{~A}-3 \mathrm{H}-6,56-58$ & 24.36 & 0.0179 & 9.0520 & 0.20 & 1.0000 & 0 & 0 & 0 & 0 & 0 & 0 & 0 & 0 & 0 & 0 & 0 & 0 & 0 & 0 & 0 & 0 & 0 \\
\hline $794 \mathrm{~A}-3 \mathrm{H}-6,94-98$ & 24.74 & 0.5728 & 23.4020 & 2.45 & 1.0000 & 2 & 0 & 0 & 90 & 41 & 0 & 22 & 2 & 76 & 0 & 0 & 0 & 0 & 0 & 0 & 0 & $\mathbf{0}$ \\
\hline $794 \mathrm{~A}-3 \mathrm{H}-6,117-119$ & 24.97 & 0.1818 & 12.9180 & 1.41 & 1.0000 & 0 & 0 & 0 & 0 & 0 & 0 & 0 & 0 & 0 & 0 & 0 & 0 & 0 & 0 & 0 & 0 & 0 \\
\hline $794 \mathrm{~A}-3 \mathrm{H}-7,24-26$ & 25.54 & 0.0152 & 8.0380 & 0.19 & 1.0000 & 0 & 0 & 0 & 0 & 0 & 0 & 0 & 0 & 0 & 0 & 0 & 0 & 0 & 0 & 0 & 0 & 0 \\
\hline $794 \mathrm{~A}-3 \mathrm{H}-7,33-35$ & 25.63 & 0.0697 & 9.8020 & 0.71 & 1.0000 & 0 & $\mathbf{0}$ & 0 & 0 & 0 & 0 & 0 & 0 & 0 & 0 & 0 & 0 & 0 & 0 & 0 & 0 & 0 \\
\hline $794 \mathrm{~A}-3 \mathrm{H}-7,54-58$ & 25.84 & 0.0342 & 18.2240 & 0.19 & 1.0000 & 0 & 0 & 0 & 0 & 0 & 0 & 0 & 0 & 0 & 0 & 0 & 0 & 0 & 0 & 0 & 0 & 0 \\
\hline $794 \mathrm{~A}-4 \mathrm{H}-1,29-32$ & 26.09 & 0.0094 & 7.6150 & 0.12 & 1.0000 & 0 & 0 & 0 & 0 & 0 & 0 & 0 & 0 & 0 & 0 & 0 & $\mathbf{0}$ & 0 & 0 & 0 & 0 & 0 \\
\hline $794 \mathrm{~A}-4 \mathrm{H}-1,65-67$ & 26.45 & 0.2063 & 14.3060 & 1.44 & 1.0000 & 0 & 0 & 0 & 0 & 0 & 0 & 0 & $\mathbf{0}$ & 0 & 0 & 0 & 0 & 0 & 0 & 0 & $\mathbf{0}$ & $\mathbf{0}$ \\
\hline $794 \mathrm{~A}-4 \mathrm{H}-1,93-99$ & 26.73 & 0.5280 & 28.3840 & 1.86 & 1.0000 & 0 & 0 & 0 & 0 & 0 & 0 & 0 & 0 & 0 & 0 & 0 & 0 & 0 & 0 & 0 & $\mathbf{0}$ & 0 \\
\hline $794 \mathrm{~A}-4 \mathrm{H}-1,117-119$ & 26.97 & 0.2016 & 12.7540 & 1.58 & 1.0000 & 0 & 0 & 0 & 0 & 0 & 0 & 0 & 0 & 0 & 0 & 0 & 0 & 0 & 0 & 0 & $\mathbf{0}$ & 0 \\
\hline $794 \mathrm{~A}-4 \mathrm{H}-2,47-49$ & 27.77 & 1.0410 & 9.5660 & 10.88 & 1.0000 & 0 & 0 & 0 & 0 & 0 & 0 & 0 & 0 & 0 & 0 & 0 & 0 & 0 & 0 & 0 & $\mathbf{0}$ & 0 \\
\hline $794 \mathrm{~A}-4 \mathrm{H}-2,74-77$ & 28.04 & 0.1622 & 8.6340 & 1.88 & 1.0000 & 0 & 0 & 0 & 0 & 0 & 0 & 0 & 0 & 0 & 0 & 0 & 0 & 0 & 0 & 0 & 0 & 0 \\
\hline $794 \mathrm{~A}-4 \mathrm{H}-2,99-105$ & 28.29 & 0.4163 & 22.4460 & 1.85 & 1.0000 & 0 & 0 & 0 & 0 & 0 & 0 & 0 & 0 & 0 & $\mathbf{0}$ & 0 & $\mathbf{0}$ & 0 & 0 & 0 & $\mathbf{0}$ & $\mathbf{0}$ \\
\hline $794 \mathrm{~A}-4 \mathrm{H}-2,144-146$ & 28.74 & 0.0318 & 10.2830 & 0.31 & 1.0000 & 0 & 0 & 0 & 0 & 0 & 0 & 0 & 0 & 0 & 0 & 0 & 0 & 0 & 0 & $\mathbf{0}$ & $\mathbf{0}$ & 0 \\
\hline $794 \mathrm{~A}-4 \mathrm{H}-3,29-31$ & 29,08 & 0.0178 & 8.2210 & 0.22 & 1.0000 & 0 & 0 & 0 & 0 & 0 & 0 & 0 & 0 & 0 & 0 & 0 & 0 & 0 & 0 & 0 & 0 & 0 \\
\hline $794 \mathrm{~A}-4 \mathrm{H}-3,64-67$ & 29.44 & 0.0541 & 9.1447 & 0.59 & 1.0000 & 0 & 0 & 0 & 0 & 0 & 0 & 0 & $\mathbf{0}$ & 0 & 0 & 0 & 0 & 0 & 0 & 0 & 0 & $\mathbf{0}$ \\
\hline $794 \mathrm{~A}-4 \mathrm{H}-3,94-100$ & 29.74 & 0.0180 & 17.6190 & 0.10 & 1.0000 & 0 & 0 & 0 & 0 & 0 & 0 & 0 & 0 & 0 & 0 & 0 & 0 & 0 & 0 & 0 & 0 & 0 \\
\hline $794 \mathrm{~A}-4 \mathrm{H}-3,142-144$ & 30.22 & 0.0279 & 9.5600 & 0.29 & 1.0000 & 0 & 0 & 0 & 0 & 0 & 0 & 0 & 0 & 0 & 0 & 0 & 0 & 0 & 0 & 0 & 0 & 0 \\
\hline $794 \mathrm{~A}-4 \mathrm{H}-4,38-41$ & 30.68 & 0.1317 & 10.6340 & 1.24 & 1.0000 & 0 & 0 & 0 & 0 & 0 & 0 & 0 & 0 & 0 & 0 & 0 & 0 & 0 & 0 & 0 & 0 & 0 \\
\hline $794 \mathrm{~A}-4 \mathrm{H}-4,47-50$ & 30.77 & 0.1843 & 11.4590 & 1.61 & 1.0000 & 0 & 0 & 0 & 0 & 0 & 0 & $\mathbf{0}$ & $\mathbf{0}$ & 0 & 0 & $\mathbf{0}$ & 0 & 0 & 0 & 0 & 0 & $\mathbf{0}$ \\
\hline $794 \mathrm{~A}-4 \mathrm{H}-4,90-95$ & 31.20 & 3.4746 & 18.9880 & 18.30 & 1.0000 & 0 & 0 & 0 & 0 & 0 & 0 & 0 & 0 & 0 & 0 & 0 & 0 & 0 & 0 & 0 & 0 & 0 \\
\hline $794 \mathrm{~A}-4 \mathrm{H}-4,132-134$ & 31.62 & 0.0440 & 9.5920 & 0.46 & 1.0000 & 0 & 0 & 0 & 0 & 0 & 0 & $\mathbf{0}$ & 0 & 0 & 0 & 0 & 0 & 0 & 0 & 0 & $\mathbf{0}$ & 0 \\
\hline $794 \mathrm{~A}-4 \mathrm{H}-5,12-15$ & 31.92 & 0.1102 & 10.9580 & 1.01 & 1.0000 & 0 & 0 & 0 & 0 & 0 & 0 & 0 & 0 & 0 & 0 & 0 & 0 & 0 & 0 & 0 & $\mathbf{0}$ & 0 \\
\hline $794 \mathrm{~A}-4 \mathrm{H}-5,46-49$ & 32.26 & 0.0307 & 9.1940 & 0.33 & 1.0000 & 0 & 0 & 0 & 0 & 0 & 0 & 0 & 0 & 0 & 0 & 0 & 0 & 0 & 0 & 0 & 0 & 0 \\
\hline $794 \mathrm{~A}-4 \mathrm{H}-5,118-121$ & 32.98 & 0.0090 & 9.0480 & 0.10 & 1.0000 & 0 & 0 & 0 & 0 & 0 & 0 & 0 & 0 & 0 & 0 & 0 & 0 & 0 & 0 & 0 & 0 & 0 \\
\hline $794 \mathrm{~A}-4 \mathrm{H}-6,17-20$ & 33.47 & 0.0036 & 9.4236 & 0.04 & 1.0000 & 0 & 0 & 0 & 0 & 0 & 0 & 0 & 0 & 0 & 0 & 0 & 0 & 0 & 0 & 0 & 0 & 0 \\
\hline $794 \mathrm{~A}-4 \mathrm{H}-6,92-94$ & 34.22 & 0.1648 & 9.3352 & 1.77 & 1.0000 & 0 & 0 & 0 & 0 & 0 & 0 & 0 & 0 & 0 & 0 & 0 & 0 & 0 & 0 & 0 & 0 & 0 \\
\hline $794 \mathrm{~A}-4 \mathrm{H}-6,95-100$ & 34.25 & 0.0000 & 22.1000 & 0.00 & 0.1563 & 89 & 0 & 0 & 90 & 306 & 0 & 65 & 7 & 26 & 1 & 0 & 0 & 0 & 0 & 0 & 0 & 0 \\
\hline $794 \mathrm{~A}-4 \mathrm{H}-6,99-102$ & 34.29 & 0.1506 & 7.5660 & 1.99 & 1.0000 & 0 & 0 & 0 & 0 & 0 & 0 & 0 & $\mathbf{0}$ & 0 & 0 & 0 & 0 & 0 & 0 & 0 & 0 & 0 \\
\hline $794 \mathrm{~A}-4 \mathrm{H}-7,32-34$ & 35.12 & 0.1281 & 14.9181 & 0.86 & 1.0000 & 0 & 0 & 0 & 0 & 0 & 0 & 0 & $\mathbf{0}$ & 0 & 0 & $\mathbf{0}$ & 0 & 0 & 0 & 0 & 0 & 0 \\
\hline $794 \mathrm{~A}-4 \mathrm{H}-7,40-42$ & 35.20 & 1.5219 & 14.5880 & 10.43 & 0.0020 & 11276 & 171 & 1 & 96 & 329 & 5 & 91 & 4 & 0 & 0 & 4 & 0 & 0 & 0 & 0 & 0 & 0 \\
\hline $794 \mathrm{~A}-5 \mathrm{H}-1,15-17$ & 35.45 & 0.2325 & 9.5742 & 2.43 & 1.0000 & 0 & 0 & 0 & 0 & 0 & 0 & 0 & 0 & 0 & 0 & 0 & 0 & 0 & 0 & 0 & 0 & 0 \\
\hline $794 \mathrm{~A}-5 \mathrm{H}-1,80-83$ & 36.10 & 0.0922 & 11.1410 & 0.83 & 1.0000 & 0 & 0 & 0 & 0 & 0 & 0 & 0 & 0 & 0 & 0 & 0 & 0 & 0 & 0 & 0 & 0 & 0 \\
\hline $794 \mathrm{~A}-5 \mathrm{H}-1,92-97$ & 36.22 & 0.4781 & 20.8400 & 2.29 & 1.0000 & 0 & 0 & 0 & 0 & 0 & 0 & 0 & 0 & 0 & 0 & 0 & 0 & 0 & 0 & 0 & 0 & 0 \\
\hline $794 \mathrm{~A}-5 \mathrm{H}-1,134-136$ & 36.64 & 0.0228 & 8.5430 & 0.27 & 1.0000 & 0 & 0 & 0 & 0 & 0 & 0 & 0 & 0 & 0 & 0 & 0 & 0 & 0 & 0 & 0 & 0 & 0 \\
\hline $794 \mathrm{~A}-5 \mathrm{H}-2,13-16$ & 36.93 & 0.0332 & 8.0790 & 0.41 & 1.0000 & 0 & 0 & 0 & 0 & 0 & 0 & 0 & 0 & 0 & 0 & 0 & 0 & 0 & 0 & 0 & 0 & 0 \\
\hline $794 \mathrm{~A}-5 \mathrm{H}-2,71-73$ & 37.51 & 0.1928 & 14.7470 & 1.31 & 1.0000 & 0 & 0 & 0 & 0 & 0 & 0 & 0 & 0 & 0 & 0 & 0 & 0 & 0 & 0 & 0 & 0 & 0 \\
\hline $794 \mathrm{~A}-5 \mathrm{H}-2,93-97$ & 37.73 & 0.4293 & 25.2900 & 1.70 & 1.0000 & 0 & 0 & 0 & 0 & 0 & 0 & 0 & 0 & 0 & 0 & 0 & 0 & 0 & 0 & 0 & 0 & 0 \\
\hline $794 \mathrm{~A}-5 \mathrm{H}-2,142-145$ & 38.22 & 0.0579 & 9.1830 & 0.63 & 1.0000 & 0 & 0 & 0 & 0 & 0 & 0 & 0 & 0 & 0 & 0 & 0 & 0 & 0 & 0 & 0 & 0 & 0 \\
\hline
\end{tabular}


Table 8 (continued).

\begin{tabular}{|c|c|c|c|c|c|c|c|c|c|c|c|c|c|c|c|c|c|c|c|c|c|c|}
\hline Sample 127. & $\begin{array}{l}\text { Depth } \\
\text { (mbsf) }\end{array}$ & $\begin{array}{c}\text { Sand } \\
\text { Weight }\end{array}$ & $\begin{array}{c}\text { Sed } \\
\text { Weight }\end{array}$ & $\begin{array}{c}\text { Sand } \\
q /\end{array}$ & Split & $\begin{array}{l}\mathrm{Pla} \\
\text { Num }\end{array}$ & $\begin{array}{l}\text { Ben } \\
\text { Num }\end{array}$ & $\begin{array}{l}\text { B/P } \\
\text { Ral }\end{array}$ & $\begin{array}{l}\text { Coil } \\
\text { Rat }\end{array}$ & $\stackrel{\#}{\mathrm{Pla}}$ & $\stackrel{\#}{\text { Ben }}$ & $\begin{array}{c}\text { PacL } \\
\%\end{array}$ & $\begin{array}{c}\text { PacR } \\
\%\end{array}$ & $\begin{array}{c}\text { Bul } \\
\%\end{array}$ & $\begin{array}{c}\text { Umb } \\
\%\end{array}$ & $\begin{array}{l}\text { Qui } \\
\stackrel{5}{t}\end{array}$ & $\underset{\%}{\text { Glu }}$ & $\underset{\%}{\operatorname{Min}}$ & $\begin{array}{l}\text { Uvu } \\
\%\end{array}$ & $\begin{array}{l}\text { Par } \\
\text { \& }\end{array}$ & $\underset{\%}{\operatorname{Hum}}$ & $\begin{array}{l}\text { Ind } \\
\%\end{array}$ \\
\hline $794 \mathrm{~A}-5 \mathrm{H}-3,27-30$ & 38.57 & 0.3220 & 8.6360 & 3.73 & 1.0000 & 0 & 0 & 0 & 0 & 0 & 0 & 0 & 0 & 0 & 0 & 0 & 0 & 0 & 0 & 0 & 0 & 0 \\
\hline $794 \mathrm{~A}-5 \mathrm{H}-3,66-68$ & 38.96 & 0.2613 & 10.1020 & 2.59 & 1.0000 & 0 & 0 & 0 & 0 & 0 & 0 & 0 & 0 & 0 & 0 & 0 & 0 & 0 & 0 & 0 & 0 & 0 \\
\hline $794 \mathrm{~A}-5 \mathrm{H}-3,93-97$ & 39.23 & 0.3170 & 25.1400 & 1.26 & 1.0000 & 0 & 0 & 0 & 0 & 0 & 0 & 0 & 0 & 0 & 0 & 0 & 0 & 0 & 0 & 0 & 0 & 0 \\
\hline $794 \mathrm{~A}-5 \mathrm{H}-3,135-137$ & 39.65 & 0.1702 & 8.0100 & 2.12 & 1.0000 & 0 & 0 & 0 & 0 & 0 & 0 & 0 & 0 & 0 & 0 & 0 & 0 & 0 & 0 & 0 & 0 & 0 \\
\hline $794 \mathrm{~A}-5 \mathrm{H}-4,8-10$ & 39.88 & 0.0042 & 7.2040 & 0.06 & 1.0000 & 0 & 0 & 0 & 0 & 0 & 0 & 0 & 0 & 0 & 0 & 0 & 0 & 0 & 0 & 0 & 0 & 0 \\
\hline $794 \mathrm{~A}-5 \mathrm{H}-4,22-25$ & 40.02 & 0.1934 & 7.3970 & 2.61 & 1.0000 & 0 & 0 & 0 & 0 & 0 & 0 & 0 & 0 & 0 & 0 & 0 & 0 & 0 & 0 & 0 & 0 & 0 \\
\hline $794 \mathrm{~A}-5 \mathrm{H}-4,54-57$ & 40.34 & 0.1360 & 13.7300 & 0.99 & 1.0000 & 0 & 0 & 0 & 0 & 0 & 0 & 0 & 0 & 0 & 0 & 0 & 0 & 0 & 0 & 0 & 0 & 0 \\
\hline $794 \mathrm{~A}-5 \mathrm{H}-4,92-97$ & 40.72 & 0.0079 & 17.5960 & 0.04 & 1.0000 & 0 & 0 & 0 & 0 & 0 & 0 & 0 & 0 & 0 & 0 & 0 & 0 & 0 & 0 & 0 & 0 & 0 \\
\hline 794A-5H-5, 93-98 & 42.23 & 0.0381 & 18.4650 & 0.21 & 1.0000 & 0 & 0 & 0 & 0 & 0 & 0 & 0 & 0 & 0 & 0 & 0 & 0 & 0 & 0 & 0 & 0 & 0 \\
\hline $794 \mathrm{~A}-5 \mathrm{H}-6,93-98$ & 43.73 & 0.0205 & 17.9360 & 0.11 & 1.0000 & 0 & 0 & 0 & 0 & 0 & 0 & 0 & 0 & 0 & 0 & 0 & 0 & 0 & 0 & 0 & 0 & 0 \\
\hline $794 \mathrm{~A}-6 \mathrm{H}-1,92-97$ & 45.72 & 0.0364 & 15.9320 & 0.23 & 1.0000 & 0 & 0 & 0 & 0 & 0 & 0 & 0 & 0 & 0 & $\mathbf{0}$ & 0 & 0 & 0 & 0 & 0 & 0 & 0 \\
\hline $794 \mathrm{~A}-6 \mathrm{H}-2,92-97$ & 47.22 & 0.1392 & 24.2810 & 0.57 & 1.0000 & 0 & 0 & 0 & 0 & 0 & 0 & 0 & 0 & 0 & 0 & 0 & 0 & 0 & 0 & 0 & 0 & 0 \\
\hline $794 \mathrm{~A}-6 \mathrm{H}-3,90-96$ & 48.70 & 0.6192 & 17.9850 & 3.44 & 1.0000 & 0 & 0 & 0 & 0 & 0 & 0 & 0 & 0 & 0 & 0 & 0 & 0 & 0 & 0 & 0 & 0 & 0 \\
\hline $794 \mathrm{~A}-6 \mathrm{H}-4,92-97$ & 50.22 & 0.0375 & 17.6260 & 0.21 & 1.0000 & 0 & 0 & 0 & 0 & 0 & 0 & 0 & 0 & 0 & 0 & 0 & 0 & 0 & 0 & 0 & 0 & 0 \\
\hline 794A-6H-5, 93-98 & 51.73 & 0.0759 & 16.9340 & 0.45 & 1.0000 & 0 & 0 & 0 & 0 & 0 & 0 & 0 & 0 & 0 & 0 & 0 & 0 & 0 & 0 & $\mathbf{0}$ & 0 & 0 \\
\hline $794 \mathrm{~A}-6 \mathrm{H}-6,84-89$ & 53.14 & 0.0000 & 25.4201 & 0.00 & 1.0000 & 0 & 0 & 0 & 0 & 0 & 0 & 0 & 0 & 0 & 0 & 0 & 0 & 0 & 0 & 0 & 0 & 0 \\
\hline $794 \mathrm{~A}-7 \mathrm{H}-1,92-96$ & 55.22 & 0.0102 & 24.7570 & 0.04 & 1.0000 & 0 & 0 & 0 & 0 & 0 & 0 & 0 & 0 & 0 & 0 & 0 & 0 & 0 & 0 & 0 & 0 & 0 \\
\hline $794 \mathrm{~A}-7 \mathrm{H}-2,94-96$ & 56.72 & 0.0069 & 27.3080 & 0.03 & 1.0000 & 0 & 0 & 0 & 0 & 0 & 0 & 0 & 0 & 0 & 0 & 0 & 0 & 0 & 0 & 0 & 0 & 0 \\
\hline $794 \mathrm{~A}-7 \mathrm{H}-3,92-96$ & 58.22 & 0.0378 & 20.8740 & 0.18 & 1.0000 & 0 & 0 & 0 & 0 & 0 & 0 & 0 & 0 & 0 & 0 & 0 & 0 & 0 & 0 & 0 & 0 & 0 \\
\hline $794 \mathrm{~A}-7 \mathrm{H}-4,92-96$ & 59,72 & 0.0505 & 0.0000 & $\ldots$ & 1.0000 & $\ldots$ & $\ldots$ & 0 & 0 & 0 & 0 & 0 & 0 & 0 & 0 & 0 & 0 & 0 & 0 & 0 & 0 & 0 \\
\hline $794 \mathrm{~A}-7 \mathrm{H}-5.92-96$ & 61.22 & 0.0197 & 20.1683 & 0.10 & 1.0000 & 0 & 0 & 0 & 0 & 0 & 0 & 0 & 0 & 0 & 0 & 0 & 0 & 0 & 0 & 0 & 0 & 0 \\
\hline $794 \mathrm{~A}-7 \mathrm{H}-6,92-96$ & 62.72 & 0.0093 & 22.5660 & 0.04 & 1.0000 & 0 & 0 & 0 & 0 & 0 & 0 & 0 & 0 & 0 & 0 & 0 & 0 & 0 & 0 & 0 & 0 & 0 \\
\hline $794 \mathrm{~A}-8 \mathrm{H}-\mathrm{I}, 93-97$ & 64.73 & 0.0197 & 16.2930 & 0.12 & 1.0000 & 0 & 0 & 0 & 0 & 0 & 0 & 0 & 0 & 0 & 0 & 0 & 0 & 0 & 0 & 0 & 0 & 0 \\
\hline $794 \mathrm{~A}-8 \mathrm{H}-2,93-97$ & 66.23 & 0.2196 & 19.0710 & 1.15 & 1.0000 & 0 & 0 & 0 & 0 & 0 & 0 & 0 & 0 & 0 & 0 & 0 & 0 & 0 & 0 & 0 & 0 & 0 \\
\hline $794 \mathrm{~A}-8 \mathrm{H}-3,93-97$ & 67.73 & 0.1284 & 19.6380 & 0.65 & 1.0000 & 0 & 0 & 0 & 0 & 0 & 0 & 0 & 0 & 0 & 0 & 0 & 0 & 0 & 0 & 0 & 0 & 0 \\
\hline $794 \mathrm{~A}-8 \mathrm{H}-4,93-97$ & 69.23 & 0.0354 & 15.8860 & 0.22 & 1.0000 & 0 & 0 & 0 & 0 & 0 & 0 & 0 & 0 & 0 & 0 & 0 & 0 & 0 & 0 & 0 & 0 & 0 \\
\hline $794 \mathrm{~A}-8 \mathrm{H}-5,93-97$ & 70.73 & 0.0174 & 14.9130 & 0.12 & 1.0000 & 0 & 0 & 0 & 0 & 0 & 0 & 0 & 0 & 0 & 0 & 0 & 0 & 0 & 0 & 0 & 0 & 0 \\
\hline $794 \mathrm{~A}-8 \mathrm{H}-6,94-98$ & 72.24 & 0.1601 & 18.6000 & 0.86 & 1.0000 & 0 & 0 & 0 & 0 & 0 & 0 & 0 & 0 & 0 & 0 & 0 & 0 & 0 & 0 & 0 & 0 & 0 \\
\hline $794 \mathrm{~A}-9 \mathrm{H}-1,93-97$ & 74.23 & 0.1392 & 14.4710 & 0.96 & 1.0000 & 0 & 0 & 0 & 0 & 0 & 0 & 0 & 0 & 0 & 0 & 0 & 0 & 0 & 0 & 0 & 0 & 0 \\
\hline $794 \mathrm{~A}-9 \mathrm{H}-2,93-97$ & 75.73 & 0.1029 & 16.4890 & 0.62 & 1.0000 & 0 & 0 & 0 & 0 & 0 & 0 & 0 & 0 & 0 & 0 & 0 & 0 & 0 & 0 & 0 & 0 & 0 \\
\hline 794A-9H-3, 93-97 & 77.23 & 0.1090 & 16.3740 & 0.67 & 1.0000 & 0 & 0 & 0 & 0 & 0 & 0 & 0 & 0 & 0 & 0 & 0 & 0 & 0 & 0 & 0 & 0 & 0 \\
\hline $794 \mathrm{~A}-9 \mathrm{H}-4,93-97$ & 78.73 & 0.1955 & 13.6440 & 1.43 & 1.0000 & 0 & 0 & 0 & 0 & 0 & 0 & 0 & 0 & 0 & 0 & 0 & 0 & 0 & 0 & $\mathbf{0}$ & 0 & 0 \\
\hline $794 \mathrm{~A}-9 \mathrm{H}-5,93-97$ & 80.23 & 0.6952 & 13.7090 & 5.07 & 1.0000 & 0 & 0 & 0 & 0 & 0 & 0 & 0 & 0 & 0 & 0 & 0 & 0 & 0 & 0 & 0 & 0 & 0 \\
\hline $794 \mathrm{~A}-9 \mathrm{H}-6,93-97$ & 81.73 & 0.8620 & 10.3830 & 8.30 & 1.0000 & 0 & 0 & 0 & 0 & 0 & 0 & 0 & 0 & 0 & 0 & 0 & 0 & 0 & 0 & 0 & 0 & 0 \\
\hline $794 \mathrm{~A}-10 \mathrm{H}-1,93-97$ & 83.73 & 0.2433 & 11.6026 & 2.10 & 1.0000 & 0 & 0 & 0 & 0 & 0 & 0 & 0 & 0 & 0 & 0 & 0 & 0 & 0 & 0 & 0 & 0 & 0 \\
\hline $794 \mathrm{~A}-10 \mathrm{H}-2,93-97$ & 85.23 & 0.1919 & 14.6610 & 1.31 & 1.0000 & 0 & 0 & 0 & 0 & 0 & 0 & 0 & 0 & 0 & 0 & 0 & 0 & 0 & 0 & 0 & 0 & 0 \\
\hline $794 \mathrm{~A}-10 \mathrm{H}-3,93-97$ & 86.73 & 0.6580 & 17.4930 & 3,76 & 1.0000 & 0 & 0 & 0 & 0 & 0 & 0 & 0 & 0 & 0 & 0 & 0 & 0 & 0 & 0 & 0 & 0 & 0 \\
\hline $794 \mathrm{~A}-10 \mathrm{H}-4,93-97$ & 88.23 & 0.9842 & 11.9200 & 8.26 & 1.0000 & 0 & 0 & 0 & 0 & 0 & 0 & 0 & 0 & 0 & 0 & 0 & 0 & 0 & 0 & 0 & 0 & 0 \\
\hline $794 \mathrm{~A}-10 \mathrm{H}-5,93-97$ & 89.73 & 0.0289 & 17.7790 & 0.16 & 1.0000 & 0 & 0 & 0 & 0 & 0 & 0 & 0 & 0 & 0 & 0 & 0 & 0 & 0 & 0 & 0 & 0 & 0 \\
\hline $794 \mathrm{~A}-10 \mathrm{H}-6,93-97$ & 91.23 & 1.1983 & 19.3350 & 6.20 & 1.0000 & 0 & 0 & 0 & 0 & 0 & 0 & 0 & 0 & 0 & 0 & 0 & 0 & 0 & 0 & 0 & 0 & 0 \\
\hline $794 \mathrm{~A}-11 \mathrm{H}-1,92-96$ & 93.22 & 0.1795 & 17.8190 & 1.01 & 1.0000 & 0 & 0 & 0 & 0 & 0 & 0 & 0 & 0 & 0 & 0 & 0 & 0 & 0 & 0 & 0 & 0 & 0 \\
\hline $794 \mathrm{~A}-11 \mathrm{H}-2,92-96$ & 94.72 & 0.4521 & 17.2420 & 2.62 & 1.0000 & 0 & 0 & 0 & 0 & 0 & 0 & 0 & 0 & 0 & 0 & 0 & 0 & 0 & 0 & 0 & 0 & 0 \\
\hline $794 \mathrm{~A}-11 \mathrm{H}-3,91-95$ & 96.21 & 0.0914 & 15.7830 & 0.58 & 1.0000 & 0 & 0 & 0 & 0 & 0 & 0 & 0 & 0 & 0 & 0 & 0 & 0 & 0 & 0 & 0 & 0 & 0 \\
\hline $794 \mathrm{~A}-11 \mathrm{H}-4,93-97$ & 97.73 & 0.2431 & 16.5090 & 1.47 & 1.0000 & 0 & 0 & 0 & 0 & 0 & 0 & 0 & 0 & 0 & 0 & 0 & 0 & 0 & 0 & 0 & 0 & 0 \\
\hline $794 \mathrm{~A}-11 \mathrm{H}-5,93-97$ & 99.23 & 0.1375 & 17.7390 & 0.78 & 1.0000 & 0 & 0 & 0 & 0 & 0 & 0 & 0 & 0 & 0 & 0 & 0 & 0 & 0 & 0 & 0 & 0 & 0 \\
\hline $794 \mathrm{~A}-11 \mathrm{H}-6,93-97$ & 100.73 & 0.4570 & 13.6480 & 3.35 & 1.0000 & 0 & 0 & 0 & 0 & 0 & 0 & 0 & 0 & 0 & 0 & 0 & 0 & 0 & 0 & 0 & 0 & 0 \\
\hline $794 \mathrm{~A}-12 \mathrm{H}-1.93-97$ & 102.73 & 0.0645 & 15.1980 & 0.42 & 1.0000 & 0 & 0 & 0 & 0 & 0 & 0 & 0 & 0 & 0 & 0 & 0 & 0 & 0 & 0 & 0 & 0 & 0 \\
\hline $794 \mathrm{~A}-12 \mathrm{H}-2,93-97$ & 104.23 & 0.1182 & 15.0920 & 0.78 & 1.0000 & 0 & 0 & 0 & 0 & 0 & 0 & 0 & 0 & 0 & 0 & 0 & 0 & 0 & 0 & 0 & 0 & 0 \\
\hline $794 \mathrm{~A}-12 \mathrm{H}-3,93-97$ & 105.73 & 0.8137 & 16.0650 & 5.07 & 1.0000 & 0 & 0 & 0 & 0 & 0 & 0 & 0 & 0 & 0 & 0 & 0 & 0 & 0 & 0 & 0 & 0 & 0 \\
\hline $794 \mathrm{~A}-12 \mathrm{H}-4,93-97$ & 107.23 & 0.8430 & 21.7810 & 3.87 & 1.0000 & 0 & 0 & 0 & 0 & 0 & 0 & 0 & 0 & 0 & 0 & 0 & 0 & 0 & 0 & 0 & 0 & 0 \\
\hline $794 \mathrm{~A}-12 \mathrm{H}-5,93-97$ & 108.73 & 0.2903 & 14.5240 & 2.00 & 1.0000 & 0 & 0 & 0 & 0 & 0 & 0 & 0 & 0 & 0 & 0 & 0 & 0 & 0 & 0 & 0 & 0 & 0 \\
\hline $794 \mathrm{~A}-12 \mathrm{H}-6,93-97$ & 110.23 & 0.1672 & 16.6970 & 1.00 & 1.0000 & 0 & 0 & 0 & 0 & 0 & 0 & 0 & 0 & 0 & 0 & 0 & 0 & 0 & 0 & 0 & 0 & 0 \\
\hline $794 \mathrm{~A}-13 \mathrm{H}-1.92-97$ & 112.22 & 0.6059 & 16.2370 & 3.73 & 1.0000 & 0 & 0 & 0 & 0 & 0 & 0 & 0 & 0 & 0 & 0 & 0 & 0 & 0 & 0 & 0 & 0 & 0 \\
\hline $794 \mathrm{~A}-13 \mathrm{H}-2,92-97$ & 113.72 & 0.2070 & 17.8700 & 1.16 & 1.0000 & 0 & 0 & 0 & 0 & 0 & 0 & 0 & 0 & 0 & 0 & 0 & 0 & 0 & 0 & 0 & 0 & 0 \\
\hline $794 \mathrm{~A}-13 \mathrm{H}-3,93-97$ & 115.22 & 0.1849 & 15.6080 & 1.18 & 1.0000 & 0 & 0 & 0 & 0 & 0 & 0 & 0 & 0 & 0 & 0 & 0 & 0 & 0 & 0 & 0 & 0 & 0 \\
\hline
\end{tabular}


Table 8 (continued).

\begin{tabular}{|c|c|c|c|c|c|c|c|c|c|c|c|c|c|c|c|c|c|c|c|c|c|c|}
\hline Sample 127. & $\begin{array}{l}\text { Depeh } \\
\text { (mbsf) }\end{array}$ & $\begin{array}{c}\text { Sand } \\
\text { Weight }\end{array}$ & $\begin{array}{c}\text { Sed } \\
\text { Weight }\end{array}$ & $\begin{array}{c}\text { Sand } \\
\%\end{array}$ & Split & $\begin{array}{l}\text { Pla } \\
\text { Num }\end{array}$ & $\begin{array}{l}\text { Ben } \\
\text { Num }\end{array}$ & $\begin{array}{l}\text { B/P } \\
\text { Rat }\end{array}$ & $\begin{array}{l}\text { Coil } \\
\text { Rat }\end{array}$ & $\begin{array}{c}\# \\
\mathrm{Pla}\end{array}$ & $\begin{array}{c}\# \\
\text { Ben }\end{array}$ & $\begin{array}{l}\text { PacL } \\
\%\end{array}$ & $\begin{array}{l}\text { PacR } \\
\%\end{array}$ & $\begin{array}{l}\text { Bul } \\
\text { \% }\end{array}$ & $\underset{\%}{\text { Umb }}$ & $\begin{array}{c}\text { Qui } \\
\%\end{array}$ & $\underset{\%}{\text { Glu }}$ & $\underset{\%}{\text { Min }}$ & $\begin{array}{l}\text { Uvu } \\
\%\end{array}$ & $\begin{array}{l}\text { Par } \\
\%\end{array}$ & $\underset{\%}{\text { Hum }}$ & $\begin{array}{l}\text { Ind } \\
\%\end{array}$ \\
\hline $794 \mathrm{~A}-13 \mathrm{H}-4.92-97$ & 116.72 & 0.0586 & 16.6000 & 0.35 & 1.0000 & 0 & 0 & 0 & 0 & 0 & 0 & 0 & 0 & 0 & 0 & 0 & 0 & 0 & 0 & 0 & 0 & 0 \\
\hline $794 \mathrm{~A}-13 \mathrm{H}-5,92-97$ & 118.22 & 0.4459 & 19.1420 & 2.33 & 1.0000 & 0 & 0 & 0 & 0 & 0 & 0 & 0 & 0 & 0 & 0 & 0 & 0 & 0 & 0 & 0 & 0 & 0 \\
\hline $794 \mathrm{~A}-13 \mathrm{H}-6.92-97$ & 119.72 & 0.0224 & 26.2900 & 0.09 & 1.0000 & 0 & 0 & 0 & 0 & 0 & 0 & 0 & 0 & 0 & 0 & 0 & 0 & 0 & 0 & 0 & 0 & 0 \\
\hline $794 \mathrm{~A}-14 \mathrm{H}-1.92-97$ & 121.72 & 0.0856 & 23.7480 & 0.36 & 1.0000 & 0 & 0 & 0 & 0 & 0 & 0 & 0 & 0 & 0 & 0 & 0 & 0 & 0 & 0 & 0 & 0 & 0 \\
\hline $794 \mathrm{~A}-14 \mathrm{H}-2,92-97$ & 123.22 & 0.0674 & 36.8020 & 0.18 & 1.0000 & 0 & 0 & 0 & 0 & 0 & 0 & 0 & 0 & 0 & 0 & 0 & 0 & 0 & 0 & 0 & 0 & 0 \\
\hline $794 \mathrm{~A}-14 \mathrm{H}-3,92-97$ & 124.72 & 0.0806 & 21.6609 & 0.37 & 1.0000 & 0 & 0 & 0 & 0 & 0 & 0 & 0 & 0 & 0 & 0 & 0 & 0 & 0 & 0 & 0 & 0 & 0 \\
\hline $794 \mathrm{~A}-14 \mathrm{H}-4,92-97$ & 126.22 & 0.2995 & 23.8909 & 1.25 & 1.0000 & 0 & 0 & 0 & 0 & 0 & 0 & 0 & 0 & 0 & 0 & 0 & 0 & 0 & 0 & 0 & 0 & 0 \\
\hline $794 \mathrm{~A}-14 \mathrm{H}-5.92-97$ & 127.72 & 0.0611 & 17.1691 & 0.36 & 1.0000 & 0 & 0 & 0 & 0 & 0 & 0 & 0 & 0 & 0 & 0 & 0 & 0 & 0 & 0 & 0 & 0 & 0 \\
\hline $794 \mathrm{~A}-14 \mathrm{H}-6,92-97$ & 129.22 & 0.1991 & 20.9693 & 0.95 & 1.0000 & 0 & 0 & 0 & 0 & 0 & 0 & 0 & 0 & 0 & 0 & 0 & 0 & 0 & 0 & 0 & 0 & 0 \\
\hline 797A-IH-I. 76-78 & 4.16 & 0.0000 & 9.4899 & 0.00 & 1.0000 & 4 & 0 & 0 & 100 & 38 & 0 & 71 & 0 & 29 & 0 & 0 & 0 & 0 & 0 & 0 & 0 & 0 \\
\hline $797 \mathrm{~A}-1 \mathrm{H}-2.76-78$ & 5.66 & 0.2384 & 5.4890 & 4.34 & 1.0000 & 4 & 0 & 4 & 86 & 22 & 1 & 86 & 14 & 0 & 0 & 0 & 0 & 0 & 0 & 0 & 0 & 0 \\
\hline $797 \mathrm{~A}-1 \mathrm{H}-3,76-78$ & 7.16 & 0.0531 & 7.4573 & 0.71 & 1.0000 & 0 & 0 & 0 & 0 & 0 & 0 & 0 & 0 & $\mathbf{0}$ & 0 & 0 & 0 & 0 & 0 & 0 & 0 & 0 \\
\hline $797 \mathrm{~A}-1 \mathrm{H}-4,76-78$ & 8.66 & 0.0109 & 4.9982 & 0.22 & 1.0000 & $\mathbf{0}$ & 0 & $\mathbf{0}$ & $\mathbf{0}$ & 0 & 0 & 0 & 0 & $\mathbf{0}$ & 0 & 0 & 0 & 0 & $\mathbf{0}$ & $\mathbf{0}$ & $\mathbf{0}$ & 0 \\
\hline $797 \mathrm{~A}-1 \mathrm{H}-5.76-78$ & 10.16 & 0.1192 & 4.5166 & 2.64 & 1.0000 & 0 & 0 & $\mathbf{0}$ & $\mathbf{0}$ & $\mathbf{0}$ & 0 & 0 & 0 & 0 & 0 & 0 & 0 & 0 & 0 & $\mathbf{0}$ & 0 & 0 \\
\hline $797 \mathrm{~B}-1 \mathrm{H}-1.76-78$ & 0.76 & 0.0835 & 8.6160 & 0.97 & 1.0000 & 0 & 0 & $\mathbf{0}$ & 0 & 0 & 0 & 0 & 0 & 0 & 0 & 0 & 0 & 0 & 0 & 0 & 0 & 0 \\
\hline 7978-1H-1, $134-136$ & 1.34 & 0.0412 & 3.3695 & 1.22 & 1.0000 & 73 & 0 & $\mathbf{0}$ & 91 & 245 & 1 & 69 & 7 & 24 & 0 & 0 & 0 & 0 & 0 & 0 & 0 & 0 \\
\hline 797B-1H-2, 13-15 & 1.63 & 0.0209 & 1.2748 & 1.64 & 0.2500 & 1174 & 69 & 6 & 96 & 374 & 22 & 91 & 3 & 1 & 0 & 1 & 1 & 0 & 2 & 1 & 0 & 0 \\
\hline $797 \mathrm{~B}-1 \mathrm{H}-2,44-46$ & 1.94 & 0.0101 & 1.6600 & 0.61 & 1.0000 & 0 & 0 & 0 & $\mathbf{0}$ & 0 & 0 & 0 & 0 & 0 & 0 & 0 & 0 & 0 & 0 & $\mathbf{0}$ & 0 & 0 \\
\hline 797B-1H-3.134-136 & 4.34 & 0.0096 & 3.0612 & 0.31 & 1.0000 & 11 & 0 & $\mathbf{0}$ & 91 & 33 & 0 & 64 & 6 & 30 & 0 & 0 & 0 & 0 & 0 & 0 & 0 & 0 \\
\hline 797B-1H-4, 74-76 & 5.24 & 0.0240 & 4.8229 & 0.50 & 1.0000 & 0 & 0 & 0 & $\mathbf{0}$ & 0 & 0 & 0 & 0 & 0 & 0 & 0 & 0 & 0 & 0 & $\mathbf{0}$ & 0 & 0 \\
\hline $797 \mathrm{~B}-1 \mathrm{H}-4,104-106$ & 5.54 & 0.0280 & 3.1292 & 0.89 & 1.0000 & 35 & 11 & 24 & 98 & 108 & 34 & 97 & 2 & 1 & 0 & 0 & 0 & 0 & 0 & $\mathbf{0}$ & 0 & 0 \\
\hline 797B-2H-1, 102-104 & 6.92 & 0.1146 & 2.7719 & 4.13 & 0.1250 & 877 & 35 & 4 & 98 & 304 & 12 & 71 & 1 & 28 & 0 & 0 & 0 & 0 & 0 & $\mathbf{0}$ & 0 & 0 \\
\hline 797B-2H-2, 76-78 & 8.16 & 0.0000 & 5.4783 & 0.00 & 1.0000 & 0 & 0 & $\mathbf{0}$ & 0 & 0 & 0 & 0 & 0 & 0 & 0 & 0 & 0 & 0 & 0 & 0 & 0 & 0 \\
\hline $797 \mathrm{~B}-2 \mathrm{H}-3.74-76$ & 9.64 & 0.2544 & 4.5686 & 5.57 & 1.0000 & 0 & 0 & $\mathbf{0}$ & $\mathbf{0}$ & 0 & 0 & 0 & $\mathbf{0}$ & 0 & 0 & 0 & 0 & 0 & 0 & $\mathbf{0}$ & 0 & 0 \\
\hline $797 \mathrm{~B}-2 \mathrm{H}-3.76-78$ & 9.66 & 0.3885 & 7.2654 & 5.35 & 1.0000 & 0 & 0 & 0 & $\mathbf{0}$ & 0 & $\mathbf{0}$ & 0 & 0 & 0 & 0 & 0 & 0 & 0 & 0 & $\mathbf{0}$ & 0 & 0 \\
\hline $797 \mathrm{~B}-2 \mathrm{H}-4,14-16$ & 10.54 & 0.0358 & 2.3550 & 1.52 & 1.0000 & 0 & 0 & 0 & $\mathbf{0}$ & 0 & 0 & 0 & 0 & 0 & 0 & 0 & 0 & 0 & 0 & 0 & 0 & 0 \\
\hline 797B-2H-4, 76-78 & 11.16 & 0.0917 & 9.3926 & 0.98 & 1.0000 & 0 & 0 & $\mathbf{0}$ & $\mathbf{0}$ & 0 & 0 & 0 & 0 & 0 & 0 & 0 & 0 & 0 & 0 & 0 & 0 & 0 \\
\hline 7978-2H-5. 76-78 & 12.66 & 0.0106 & 5.9251 & 0.18 & 1.0000 & 0 & 0 & 0 & 0 & 0 & 0 & 0 & 0 & 0 & 0 & 0 & 0 & 0 & 0 & 0 & 0 & 0 \\
\hline $797 \mathrm{~B}-2 \mathrm{H}-6,76-78$ & 14.16 & 0.4081 & 8.5711 & 4.76 & 1.0000 & 0 & 0 & 0 & 0 & 0 & 0 & 0 & 0 & 0 & 0 & 0 & 0 & 0 & 0 & $\mathbf{0}$ & 0 & 0 \\
\hline 797B-2H-6. 134-136 & 14.74 & 0.1101 & 2.0578 & 5.35 & 1.0000 & 155 & 0 & 0 & 98 & 319 & 0 & 83 & 2 & 0 & 0 & 1 & 0 & 0 & 4 & 10 & 0 & 0 \\
\hline 797B-3H-1, 77-79 & 16.17 & 0.0891 & 10.3619 & 0.86 & 1.0000 & 0 & 0 & $\mathbf{0}$ & 0 & 0 & 0 & 0 & 0 & 0 & 0 & 0 & 0 & 0 & 0 & 0 & 0 & 0 \\
\hline $797 \mathrm{~B}-3 \mathrm{H}-2,77-79$ & 17.67 & 0.0387 & 7.2258 & 0.54 & 1.0000 & 2 & 0 & $\mathbf{0}$ & 20 & 13 & 0 & 8 & 31 & 54 & 0 & 8 & 0 & 0 & 0 & 0 & 0 & 0 \\
\hline 797B-3H-3, 77-79 & 19.17 & 0.1350 & 7.1800 & 1.88 & 1.0000 & 0 & 0 & $\mathbf{0}$ & 0 & 0 & 0 & 0 & 0 & 0 & 0 & 0 & 0 & 0 & 0 & 0 & 0 & 0 \\
\hline 797B-3H-4. 77-79 & 20.67 & 0.0350 & 6.1081 & 0.57 & 1.0000 & 0 & 0 & 0 & 0 & 0 & 0 & 0 & 0 & 0 & 0 & 0 & 0 & 0 & 0 & 0 & 0 & 0 \\
\hline 797B-3H-5. 77-79 & 22.17 & 0.1702 & 7.1258 & 2.39 & 1.0000 & 0 & 0 & 0 & 0 & 0 & 0 & 0 & 0 & 0 & 0 & 0 & 0 & 0 & 0 & 0 & 0 & 0 \\
\hline 797B-3H-6, 77-79 & 23.67 & 0.0999 & 7.5471 & 1.32 & 1.0000 & $\mathbf{0}$ & 0 & 0 & $\mathbf{0}$ & 0 & 0 & 0 & 0 & 0 & 0 & 0 & 0 & 0 & 0 & 0 & $\mathbf{0}$ & 0 \\
\hline 797B-3H-6, 103-105 & 23.93 & 0.0093 & 3.8248 & 0.24 & 1.0000 & 0 & 0 & $\mathbf{0}$ & $\mathbf{0}$ & 0 & 0 & 0 & 0 & 0 & 0 & 0 & 0 & 0 & 0 & 0 & 0 & 0 \\
\hline 797B-3H-6. 135- 137 & 24.25 & 0.1371 & 3.5552 & 3.86 & 0.0156 & 12585 & 108 & 1 & 97 & 698 & 6 & 77 & 2 & 12 & 0 & 5 & 0 & 0 & 0 & 3 & 0 & 1 \\
\hline $797 \mathrm{~B}-3 \mathrm{H}-7,14-16$ & 24.54 & 0.0799 & 3.7423 & 2.14 & 0.0156 & 8428 & 1593 & 16 & 95 & 492 & 93 & 32 & 2 & 0 & 0 & 7 & 4 & 1 & 0 & 52 & 0 & 2 \\
\hline 797B-4H-1.77-79 & 25.67 & 0.0327 & 5,4682 & 0.60 & 1.0000 & 0 & 0 & 0 & 0 & 0 & 0 & 0 & 0 & $\mathbf{0}$ & 0 & 0 & 0 & 0 & 0 & 0 & 0 & 0 \\
\hline $797 \mathrm{~B}-4 \mathrm{H}-2,20-22$ & 26.60 & 0.0027 & 2.8818 & 0.09 & 1.0000 & 0 & 0 & 0 & $\mathbf{0}$ & 0 & 0 & 0 & 0 & $\mathbf{0}$ & 0 & 0 & 0 & 0 & 0 & 0 & 0 & 0 \\
\hline $797 \mathrm{~B}-4 \mathrm{H}-2,77-79$ & 27.17 & 0.0439 & 4.9804 & 0.88 & 1.0000 & 0 & 0 & $\mathbf{0}$ & $\mathbf{0}$ & 0 & 0 & 0 & 0 & 0 & 0 & 0 & 0 & 0 & 0 & 0 & $\mathbf{0}$ & 0 \\
\hline $797 \mathrm{~B}-4 \mathrm{H}-3,44-46$ & 28.34 & 0.0353 & 0.7667 & 4.60 & 1.0000 & 50 & 38 & 43 & 80 & 38 & 29 & 11 & 3 & 21 & 0 & 50 & 0 & 0 & 16 & 0 & 0 & 0 \\
\hline $797 \mathrm{~B}-4 \mathrm{H}-3,77-79$ & 28.67 & 0.0571 & 4.9549 & 1.15 & 1.0000 & 54 & 0 & 0 & 75 & 268 & 0 & 1 & 0 & 99 & 0 & 0 & 0 & 0 & 0 & 0 & 0 & 0 \\
\hline $797 \mathrm{~B}-4 \mathrm{H}-3,102-104$ & 28.92 & 0.0838 & 3.2582 & 2.57 & 0.0313 & 3344 & 1255 & 27 & 56 & 341 & 128 & 1 & 1 & 80 & 0 & 16 & 0 & 0 & 1 & 1 & 0 & 0 \\
\hline $797 \mathrm{~B}-4 \mathrm{H}-3,135-137$ & 29.25 & 0.8097 & 3.2370 & 25.01 & 0.0469 & 1943 & 198 & 9 & 98 & 295 & 30 & 74 & 1 & 8 & 0 & 16 & 0 & 0 & 1 & 1 & 0 & 0 \\
\hline $797 \mathrm{~B}-4 \mathrm{H}-4,13-15$ & 29.53 & 0.0093 & 3.1689 & 0.29 & 1.0000 & 11 & 23 & 68 & 100 & 35 & 73 & 100 & 0 & 0 & 0 & 0 & 0 & 0 . & 0 & 0 & 0 & 0 \\
\hline $797 \mathrm{~B}-4 \mathrm{H}-4,77-79$ & 30.17 & 0.0661 & 8.1956 & 0.81 & 1.0000 & 0 & 0 & 0 & 0 & 0 & 0 & 0 & 0 & 0 & 0 & 0 & 0 & 0 & 0 & 0 & 0 & 0 \\
\hline $797 \mathrm{~B}-4 \mathrm{H}-4,102-104$ & 30.42 & 0.0721 & 2.3006 & 3.13 & 0.0781 & 1864 & 189 & 9 & 80 & 335 & 34 & 6 & 1 & 87 & 2 & 3 & 0 & 0 & 0 & 0 & 0 & 0 \\
\hline $797 \mathrm{~B}-4 \mathrm{H}-4,135-137$ & 30.75 & 0.2588 & 1.7763 & 14.57 & 0.0078 & 32407 & 144 & 0 & 98 & 449 & 2 & 72 & 1 & 21 & 1 & 3 & 0 & 0 & 1 & 1 & 0 & 0 \\
\hline $797 \mathrm{~B}-4 \mathrm{H}-5,43-45$ & 31.33 & 0.1124 & 1.6416 & 6.85 & 0.0234 & 8304 & 234 & 3 & 98 & 319 & 9 & 75 & 2 & 20 & 0 & 1 & 0 & 0 & 0 & 0 & 0 & 2 \\
\hline $797 \mathrm{~B}-4 \mathrm{H}-5,74-76$ & 31.64 & 0.0193 & 5.7512 & 0.34 & 1.0000 & 0 & 0 & $\mathbf{0}$ & 0 & 2 & $\mathbf{0}$ & 0 & 0 & 100 & 0 & 0 & 0 & 0 & 0 & 0 & 0 & 0 \\
\hline $797 \mathrm{~B}-4 \mathrm{H}-6,77-79$ & 33.17 & 0.0300 & 3.3954 & 0.88 & 1.0000 & 0 & 0 & 0 & 0 & 0 & 0 & 0 & 0 & 0 & 0 & 0 & 0 & 0 & 0 & 0 & 0 & 0 \\
\hline $797 \mathrm{~B}-4 \mathrm{H}-6,135-137$ & 33.75 & 0.0319 & 3.3955 & 0.94 & 0.0625 & 2837 & 19 & 1 & 96 & 602 & 4 & 82 & 4 & 3 & 0 & 11 & 0 & 0 & 0 & 0 & 0 & 0 \\
\hline 797B-5H-1, 78-80 & 35.18 & 0.0927 & 9.5903 & 0.97 & 1.0000 & 9 & 0 & 0 & 93 & 83 & 0 & 52 & 4 & 45 & 0 & 0 & 0 & 0 & 0 & 0 & 0 & 0 \\
\hline
\end{tabular}


Table 8 (continued),

\begin{tabular}{|c|c|c|c|c|c|c|c|c|c|c|c|c|c|c|c|c|c|c|c|c|c|c|}
\hline Sample 127. & $\begin{array}{l}\text { Depth } \\
\text { (mbsf) }\end{array}$ & $\begin{array}{c}\text { Sand } \\
\text { Weight }\end{array}$ & $\begin{array}{c}\text { Sed } \\
\text { Weight }\end{array}$ & $\underset{c \%}{\text { Sand }}$ & Split & $\begin{array}{c}\mathrm{Pla} \\
\text { Num }\end{array}$ & $\begin{array}{l}\text { Ben } \\
\text { Num }\end{array}$ & $\begin{array}{l}\text { B/P } \\
\text { Rat }\end{array}$ & $\begin{array}{l}\text { Coil } \\
\text { Rat }\end{array}$ & $\begin{array}{c}\text { \# } \\
\text { Pla }\end{array}$ & $\begin{array}{c}\# \\
\text { Ben }\end{array}$ & $\begin{array}{c}\text { Pacl. } \\
\text { \% }\end{array}$ & $\begin{array}{c}\text { PacR } \\
\%\end{array}$ & $\begin{array}{c}\text { Bul } \\
\qquad\end{array}$ & $\underset{\%}{\text { Umb }}$ & $\begin{array}{l}\text { Qui } \\
\text { \% }\end{array}$ & $\begin{array}{c}\text { Glu } \\
\%\end{array}$ & $\underset{\%}{\operatorname{Min}}$ & $\begin{array}{l}\text { Uvu } \\
\text { \% }\end{array}$ & $\begin{array}{l}\text { Par } \\
\%\end{array}$ & $\underset{\%}{\text { Hum }}$ & $\begin{array}{l}\text { Ind } \\
\%\end{array}$ \\
\hline $797 \mathrm{~B}-5 \mathrm{H}-1,132-134$ & 35.72 & 0.0666 & 3.9071 & 1.70 & 0.0938 & 881 & 134 & 13 & 98 & 323 & 49 & 46 & I & 49 & 4 & 0 & 0 & 0 & 0 & 0 & $\mathbf{0}$ & 0 \\
\hline $797 \mathrm{~B}-5 \mathrm{H}-2,44-46$ & 36.34 & 0.0966 & 2.4283 & 3.98 & 1.0000 & 0 & 0 & 0 & 0 & 0 & 0 & 0 & 0 & 0 & 0 & 0 & 0 & 0 & 0 & 0 & $\mathbf{0}$ & 0 \\
\hline $797 \mathrm{~B}-5 \mathrm{H}-2,78-80$ & 36.68 & 0.2993 & 8.8424 & 3.38 & 1.0000 & 0 & 0 & 0 & 0 & 0 & 0 & 0 & 0 & 0 & 0 & 0 & 0 & 0 & 0 & 0 & 0 & 0 \\
\hline $797 \mathrm{~B}-5 \mathrm{H}-2,103-105$ & 36.93 & 0.0403 & 3.0209 & 1.33 & 1.0000 & 113 & 19 & 14 & 87 & 340 & 56 & 43 & 6 & 48 & $\mathbf{0}$ & 3 & 0 & 0 & 0 & 0 & 0 & 0 \\
\hline 797B-5H-3, 78-80 & 38.18 & 0.0298 & 6.2512 & 0.48 & 1.0000 & 0 & 0 & 0 & 0 & 0 & 0 & 0 & 0 & 0 & 0 & 0 & 0 & 0 & 0 & 0 & 0 & 0 \\
\hline $797 \mathrm{~B}-5 \mathrm{H}-4,43-45$ & 39.33 & 0.0366 & 4.2421 & 0.86 & 1.0000 & 0 & 0 & 0 & 0 & 0 & 0 & 0 & 0 & 0 & 0 & 0 & 0 & 0 & 0 & 0 & 0 & 0 \\
\hline 797B-5H-4, 74-76 & 39.64 & 0.0309 & 3.8578 & 0.80 & 1.0000 & 61 & 1 & 1 & 98 & 237 & 2 & 95 & 2 & 3 & 0 & 0 & 0 & 0 & 0 & $\mathbf{0}$ & 0 & $\mathbf{0}$ \\
\hline 797B-5H-4. 78-80 & 39.68 & 1.5850 & 10.8650 & 14.59 & 0.0020 & 17073 & 46 & 0 & 97 & 371 & 1 & 56 & 2 & 41 & 0 & 1 & 0 & 0 & 0 & 0 & 0 & 0 \\
\hline 797B-5H-5, 11-13 & 40.51 & 0.0214 & 2.7329 & 0.78 & 0.5000 & 544 & 50 & 8 & 98 & 744 & 68 & 87 & 2 & 4 & 0 & 6 & 0 & 0 & 0 & 0 & 0 & 0 \\
\hline $797 \mathrm{~B}-5 \mathrm{H}-5.43-45$ & 40.83 & 0.0330 & 2.8448 & 1.16 & 1.0000 & 51 & 73 & 59 & 100 & 144 & 209 & 98 & 0 & 1 & 0 & 1 & 0 & 0 & 1 & 0 & 0 & 0 \\
\hline 797B-5H-5, 74-76 & 41.14 & 0.0284 & 3.5086 & 0.81 & 1.0000 & 0 & 0 & 0 & 0 & 0 & 0 & 0 & 0 & 0 & 0 & 0 & 0 & 0 & 0 & 0 & 0 & 0 \\
\hline 797B-5H-5. 78-80 & 41.18 & 7.1045 & 9.7598 & 72.79 & 1.0000 & 0 & 0 & 0 & 0 & 0 & 0 & 0 & 0 & 0 & 0 & 0 & 0 & 0 & 0 & 0 & 0 & 0 \\
\hline 797B-5H-5, 13I-133 & 41.71 & 0.0396 & 2.7038 & 1.46 & 0.0469 & 2689 & 197 & 7 & 81 & 341 & 25 & 70 & 16 & 9 & 0 & 3 & 0 & $\mathbf{0}$ & 1 & 1 & 0 & 1 \\
\hline 797B-5H-6, 14-16 & 42.04 & 0.1587 & 3.2753 & 4.85 & 1.0000 & 0 & 0 & 0 & 0 & 0 & 0 & 0 & 0 & 0 & 0 & 0 & 0 & 0 & 0 & $\mathbf{0}$ & 0 & 0 \\
\hline 797B-5H-6, 78-80 & 42.68 & 0.2103 & 13.7800 & 1.53 & 1.0000 & 0 & 0 & 0 & 0 & 0 & 0 & 0 & 0 & 0 & 0 & 0 & 0 & 0 & 0 & 0 & 0 & 0 \\
\hline $797 \mathrm{~B}-5 \mathrm{H}-6,102-104$ & 42.92 & 0.2136 & 2.6603 & 8.03 & 0.0156 & 10048 & 1349 & 12 & 96 & 417 & 56 & 71 & 3 & 4 & 0 & 15 & 0 & 0 & 2 & 3 & 0 & 1 \\
\hline 797B-5H-7, 14-16 & 43.54 & 0.0553 & 2.6670 & 2.07 & 1.0000 & 0 & 0 & 0 & 0 & 0 & 0 & 0 & 0 & 0 & 0 & 0 & 0 & 0 & 0 & 0 & 0 & 0 \\
\hline $797 \mathrm{~B}-5 \mathrm{H}-7,43-45$ & 43.83 & 0.0725 & 3.5938 & 2.02 & 0.0625 & 1429 & 31 & 2 & 87 & 321 & 7 & 56 & 8 & 20 & 8 & 7 & 0 & 0 & 0 & 0 & 0 & 0 \\
\hline $797 \mathrm{~B}-6 \mathrm{H}-1,78-80$ & 44.68 & 0.0614 & 6.6945 & 0.92 & 1.0000 & 0 & 0 & 0 & 0 & 0 & 0 & 0 & 0 & 0 & 0 & 0 & 0 & 0 & 0 & 0 & 0 & 0 \\
\hline $797 \mathrm{~B}-6 \mathrm{H}-1,132-134$ & 45.22 & 0.1504 & 3.2690 & 4.60 & 0.1250 & 110 & 5 & 4 & 100 & 45 & 2 & 73 & 0 & 0 & 13 & 0 & 0 & 0 & 0 & 0 & 0 & 13 \\
\hline $797 \mathrm{~B}-6 \mathrm{H}-2,74-76$ & 46.14 & 0.0427 & 4.2680 & 1.00 & 0.1250 & 609 & 6 & 1 & 90 & 325 & 3 & 70 & 8 & 0 & 0 & 10 & 0 & 0 & 0 & 0 & 0 & 12 \\
\hline $797 \mathrm{~B}-6 \mathrm{H}-2,78-80$ & 46.18 & 0.1505 & 7.4428 & 2.02 & 0.0234 & 2107 & 17 & 1 & 94 & 367 & 3 & 90 & 6 & 1 & 0 & 1 & 0 & 0 & 0 & 0 & 0 & 2 \\
\hline $797 \mathrm{~B}-6 \mathrm{H}-2,102-104$ & 46.42 & 0.0918 & 4.7140 & 1.95 & 0.1250 & 608 & 15 & 2 & 92 & 358 & 9 & 82 & 8 & 1 & 0 & 3 & 0 & 0 & 0 & 0 & 0 & 7 \\
\hline $797 \mathrm{~B}-6 \mathrm{H}-3,13-15$ & 47.03 & 0.0087 & 2.9910 & 0.29 & 1.0000 & 0 & 0 & 0 & 0 & 0 & 0 & 0 & 0 & 0 & 0 & 0 & 0 & 0 & 0 & 0 & 0 & 0 \\
\hline $797 \mathrm{~B}-6 \mathrm{H}-3,74-76$ & 47.64 & 0.2587 & 2.8960 & 8.93 & 0.0313 & 3961 & 177 & 4 & 80 & 359 & 16 & 62 & 16 & 15 & 0 & 0 & 0 & 0 & 0 & 0 & 0 & 7 \\
\hline $797 \mathrm{~B}-6 \mathrm{H}-3,78-80$ & 47.68 & 0.0746 & 7.8028 & 0.96 & 0.0625 & 687 & 14 & 2 & 85 & 335 & 7 & 84 & 14 & 0 & 0 & 1 & 0 & 0 & 0 & 0 & 0 & 1 \\
\hline $797 \mathrm{~B}-6 \mathrm{H}-3,102-104$ & 47.92 & 0.1002 & 2.9110 & 3.44 & 0.0234 & 5197 & 132 & 2 & 91 & 354 & 9 & 87 & 9 & 0 & 0 & 0 & 0 & 0 & 0 & 0 & 0 & 4 \\
\hline $797 \mathrm{~B}-6 \mathrm{H}-4,78-80$ & 49.18 & 0.0725 & 0.9804 & 7.39 & 1.0000 & 0 & 0 & 0 & 0 & 0 & 0 & 0 & 0 & $\mathbf{0}$ & 0 & 0 & 0 & 0 & 0 & 0 & 0 & 0 \\
\hline $797 \mathrm{~B}-6 \mathrm{H}-5,78-80$ & 50.68 & 0.3409 & 6.1834 & 5.51 & 1.0000 & 0 & 0 & 0 & 0 & 0 & 0 & 0 & 0 & 0 & 0 & 0 & 0 & 0 & 0 & 0 & 0 & 0 \\
\hline $797 \mathrm{~B}-6 \mathrm{H}-6,78-80$ & 52.18 & 0.2606 & 7.5699 & 3.44 & 1.0000 & 0 & 0 & 0 & 0 & 0 & 0 & $\mathbf{0}$ & 0 & $\mathbf{0}$ & $\mathbf{0}$ & 0 & 0 & 0 & 0 & 0 & $\mathbf{0}$ & 0 \\
\hline 797B-7H-1, 78-80 & 54.18 & 0.1997 & 12.9515 & 1.54 & 1.0000 & 0 & 0 & 0 & 0 & 0 & 0 & 0 & 0 & 0 & 0 & 0 & 0 & 0 & 0 & 0 & 0 & 0 \\
\hline 797B-7H-2, 78-80 & 55.68 & 0.0524 & 7.0757 & 0,74 & 1.0000 & 0 & 0 & 0 & 0 & 0 & 0 & 0 & 0 & 0 & 0 & 0 & 0 & 0 & 0 & 0 & 0 & 0 \\
\hline 797B-7H-3, 78-80 & 57.18 & 0.0914 & 9.8832 & 0.92 & 1.0000 & 0 & 0 & 0 & 0 & 0 & 0 & 0 & 0 & 0 & 0 & 0 & 0 & 0 & 0 & 0 & 0 & 0 \\
\hline 797B-7H-4, 78-80 & 58.68 & 0.0503 & 8.8535 & 0.57 & 1.0000 & 0 & $\mathbf{0}$ & 0 & 0 & 0 & 0 & 0 & 0 & 0 & $\mathbf{0}$ & 0 & 0 & 0 & 0 & 0 & $\mathbf{0}$ & 0 \\
\hline 797B-7H-5, 78-80 & 60.18 & 0.0328 & 11.5727 & 0.28 & 1.0000 & 0 & 0 & 0 & 0 & 0 & 0 & 0 & 0 & 0 & 0 & 0 & 0 & 0 & 0 & 0 & 0 & 0 \\
\hline 797B-7H-6, 78-80 & 61.68 & 0.0482 & 10.4509 & 0.46 & 1.0000 & 0 & 0 & 0 & 0 & 0 & 0 & 0 & 0 & 0 & 0 & 0 & 0 & 0 & 0 & $\mathbf{0}$ & 0 & 0 \\
\hline $797 \mathrm{~B}-8 \mathrm{H}-1,78-80$ & 63.68 & 0.0566 & 8,9761 & 0.63 & 1.0000 & 0 & 0 & 0 & 0 & 0 & 0 & 0 & 0 & 0 & 0 & 0 & 0 & 0 & 0 & 0 & $\mathbf{0}$ & 0 \\
\hline $797 \mathrm{~B}-8 \mathrm{H}-2,78-80$ & 65.18 & 0.0405 & 9.8936 & 0.41 & 1.0000 & 0 & 0 & 0 & 0 & 0 & 0 & 0 & 0 & 0 & 0 & 0 & 0 & 0 & 0 & $\mathbf{0}$ & 0 & 0 \\
\hline $797 \mathrm{~B}-8 \mathrm{H}-3,78-80$ & 66.68 & 0.0037 & 5.9682 & 0.06 & 1.0000 & 0 & 0 & 0 & 0 & 0 & 0 & 0 & 0 & 0 & 0 & 0 & 0 & 0 & 0 & 0 & 0 & 0 \\
\hline $797 \mathrm{~B}-8 \mathrm{H}-4,77-79$ & 68.17 & 0.0150 & 7.4428 & 0.20 & 1.0000 & 0 & 0 & 0 & 0 & 0 & 0 & 0 & 0 & 0 & 0 & 0 & 0 & 0 & 0 & 0 & 0 & 0 \\
\hline $797 \mathrm{~B}-8 \mathrm{H}-5,77-79$ & 69.67 & 0.0172 & 5.6497 & 0.30 & 1.0000 & 0 & 0 & 0 & 0 & 0 & 0 & 0 & 0 & 0 & 0 & 0 & 0 & 0 & 0 & 0 & 0 & $\mathbf{0}$ \\
\hline $797 \mathrm{~B}-8 \mathrm{H}-6,78-80$ & 71.18 & 0.0099 & 6.6763 & 0.15 & 1.0000 & 0 & 0 & 0 & 0 & 0 & 0 & 0 & 0 & 0 & 0 & 0 & 0 & 0 & 0 & 0 & 0 & 0 \\
\hline $797 \mathrm{~B}-9 \mathrm{H}-1,78-80$ & 73.18 & 0.1971 & 8.0221 & 2.46 & 1,0000 & 0 & 0 & 0 & 0 & 0 & 0 & 0 & 0 & 0 & 0 & 0 & 0 & 0 & 0 & 0 & 0 & 0 \\
\hline $797 \mathrm{~B}-9 \mathrm{H}-2,78-80$ & 74.68 & 0.1170 & 15.8724 & 0.74 & 1.0000 & 0 & 0 & 0 & 0 & 0 & 0 & 0 & 0 & 0 & 0 & 0 & 0 & 0 & 0 & 0 & 0 & 0 \\
\hline $797 \mathrm{~B}-9 \mathrm{H}-3,78-80$ & 76.18 & 0.1912 & 10.3306 & 1.85 & 1.0000 & 0 & 0 & 0 & 0 & 0 & 0 & 0 & 0 & 0 & 0 & 0 & 0 & 0 & 0 & 0 & 0 & 0 \\
\hline 797B-9H-4, 78-80 & 77.68 & 0.0544 & 7.2795 & 0.75 & 1.0000 & 0 & 0 & 0 & 0 & 0 & 0 & 0 & 0 & 0 & 0 & 0 & 0 & 0 & 0 & 0 & 0 & 0 \\
\hline 797B-9H-5, 78-80 & 79.18 & 0.0091 & 9.7021 & 0.09 & 1.0000 & 0 & 0 & 0 & 0 & 0 & 0 & 0 & 0 & 0 & 0 & 0 & 0 & 0 & 0 & 0 & 0 & 0 \\
\hline 797B-9H-6. 78-80 & 80.68 & 0.0199 & 8.9368 & 0.22 & 1.0000 & 0 & 0 & 0 & 0 & 0 & 0 & 0 & 0 & 0 & 0 & 0 & 0 & 0 & 0 & 0 & 0 & 0 \\
\hline $797 \mathrm{~B}-10 \mathrm{H}-1,78-80$ & 82.68 & 0.0276 & 10.0406 & 0.27 & 1.0000 & 0 & 0 & 0 & 0 & 0 & 0 & 0 & 0 & 0 & 0 & 0 & 0 & 0 & 0 & 0 & 0 & 0 \\
\hline $797 \mathrm{~B}-1 \mathrm{OH}-2,78-80$ & 84.18 & 0.0519 & 13.4138 & 0.39 & 0.1250 & 361 & 1 & 0 & 63 & 606 & 2 & 23 & 13 & 27 & 0 & 24 & 0 & 1 & 4 & 7 & 0 & 1 \\
\hline $797 \mathrm{~B}-10 \mathrm{H}-3,78-80$ & 85.68 & 0.0131 & 9.1135 & 0.14 & 1.0000 & 0 & 0 & 0 & 0 & 0 & 0 & 0 & 0 & 0 & 0 & 0 & 0 & 0 & $\mathbf{0}$ & $\mathbf{0}$ & 0 & 0 \\
\hline $797 \mathrm{~B}-10 \mathrm{H}-4,78-80$ & 87.18 & 0.0284 & 8.5812 & 0.33 & 1.0000 & 0 & 0 & 0 & 0 & 0 & 0 & 0 & 0 & 0 & 0 & 0 & 0 & 0 & 0 & 0 & 0 & 0 \\
\hline $797 \mathrm{~B}-10 \mathrm{H}-5,78-80$ & 88.68 & 0.1155 & 15.4300 & 0.75 & 1.0000 & 0 & 0 & 0 & 0 & 0 & 0 & 0 & 0 & 0 & 0 & 0 & 0 & 0 & 0 & 0 & 0 & 0 \\
\hline $797 \mathrm{~B}-10 \mathrm{H}-6,78-80$ & 90.18 & 0.0697 & 8.8998 & 0.78 & 1.0000 & 0 & 0 & 0 & 0 & 0 & 0 & 0 & 0 & 0 & 0 & 0 & 0 & 0 & 0 & 0 & 0 & 0 \\
\hline $797 \mathrm{~B}-11 \mathrm{H}-1,78-80$ & 92.18 & 0.0981 & 8.0499 & 1.22 & 1.0000 & 0 & 0 & 0 & 0 & 0 & 0 & 0 & 0 & 0 & 0 & 0 & 0 & 0 & 0 & 0 & 0 & 0 \\
\hline
\end{tabular}


Table 8 (continued).

\begin{tabular}{|c|c|c|c|c|c|c|c|c|c|c|c|c|c|c|c|c|c|c|c|c|c|c|}
\hline Sample 127- & $\begin{array}{l}\text { Depth } \\
\text { (mbsf) }\end{array}$ & $\begin{array}{c}\text { Sand } \\
\text { Weight }\end{array}$ & $\begin{array}{c}\text { Sed } \\
\text { Weight }\end{array}$ & $\begin{array}{c}\text { Sand } \\
\%\end{array}$ & Split & $\begin{array}{c}\mathrm{Pla} \\
\mathrm{Num}\end{array}$ & $\begin{array}{l}\text { Ben } \\
\text { Num }\end{array}$ & $\begin{array}{l}\text { B/P } \\
\text { Rat }\end{array}$ & $\begin{array}{l}\text { Coil } \\
\text { Rat }\end{array}$ & $\begin{array}{c}\# \\
\mathrm{Pla}\end{array}$ & $\stackrel{\#}{B e n}$ & $\begin{array}{c}\text { PacL } \\
\%\end{array}$ & $\begin{array}{c}\text { PacR } \\
\%\end{array}$ & $\begin{array}{c}\text { Bul } \\
\tilde{F}\end{array}$ & $\underset{\%}{\text { Umb }}$ & $\begin{array}{l}\text { Qui } \\
\%\end{array}$ & $\begin{array}{c}\text { Glu } \\
\%\end{array}$ & $\begin{array}{c}\text { Min } \\
\mathscr{5}\end{array}$ & $\begin{array}{c}\text { Uvu } \\
\%\end{array}$ & $\begin{array}{c}\text { Par } \\
\%\end{array}$ & $\underset{\%}{\text { Hum }}$ & $\begin{array}{l}\text { Ind } \\
\%\end{array}$ \\
\hline $797 \mathrm{~B}-11 \mathrm{H}-2,78-80$ & 93.68 & 0.0935 & 9.6681 & 0.97 & 1.0000 & 0 & 0 & 0 & 0 & 0 & 0 & 0 & 0 & 0 & 0 & 0 & 0 & 0 & 0 & 0 & 0 & 0 \\
\hline $797 \mathrm{~B}-11 \mathrm{HH}-3.78-80$ & 95.18 & 0.0620 & 7.5263 & 0.82 & 1.0000 & 0 & 0 & 0 & 0 & 0 & 0 & 0 & 0 & 0 & 0 & 0 & 0 & 0 & 0 & 0 & 0 & 0 \\
\hline $797 \mathrm{~B}-11 \mathrm{H}-4,78-80$ & 96.68 & 0.0540 & 8.3024 & 0.65 & 1.0000 & 0 & 0 & 0 & 0 & 0 & 0 & 0 & 0 & 0 & $\mathbf{0}$ & 0 & 0 & $\mathbf{0}$ & 0 & 0 & 0 & 0 \\
\hline 797B-11H-5, 78-80 & 98.18 & 0.0984 & 8.5625 & 1.15 & 1.0000 & 0 & 0 & 0 & 0 & 0 & 0 & 0 & 0 & 0 & 0 & 0 & 0 & 0 & 0 & 0 & 0 & 0 \\
\hline $797 \mathrm{~B}-11 \mathrm{H}-6.78-80$ & 99.68 & 0.1742 & 8.5208 & 2.04 & 1.0000 & 0 & 0 & 0 & 0 & 0 & 0 & 0 & 0 & 0 & 0 & 0 & 0 & 0 & 0 & 0 & 0 & 0 \\
\hline $797 \mathrm{~B}-12 \mathrm{H}-1.78-80$ & 101.68 & 0.2559 & 7.7430 & 3.30 & 1.0000 & 0 & 0 & 0 & 0 & 0 & 0 & 0 & 0 & 0 & $\mathbf{0}$ & 0 & $\mathbf{0}$ & 0 & 0 & 0 & 0 & 0 \\
\hline $797 \mathrm{~B}-12 \mathrm{H}-2,78-80$ & 103.18 & 0.0062 & 6.4132 & 0.10 & 1.0000 & 0 & 0 & 0 & 0 & 0 & 0 & 0 & 0 & 0 & 0 & 0 & 0 & 0 & 0 & 0 & 0 & 0 \\
\hline $797 \mathrm{~B}-12 \mathrm{H}-3,78-80$ & 104.68 & 0.0326 & 6.6460 & 0.49 & 1.0000 & 0 & 0 & 0 & 0 & 0 & 0 & 0 & 0 & 0 & 0 & 0 & 0 & 0 & 0 & 0 & 0 & 0 \\
\hline $797 \mathrm{~B}-12 \mathrm{H}-4,78-80$ & 106.18 & 0.0635 & 7,6648 & 0.83 & 1.0000 & 0 & 0 & 0 & 0 & 0 & 0 & 0 & 0 & 0 & 0 & 0 & $\mathbf{0}$ & 0 & 0 & 0 & 0 & 0 \\
\hline $797 \mathrm{~B}-12 \mathrm{H}-5,78-80$ & 107.68 & 0.0998 & 6.3714 & 1.57 & 1,0000 & 0 & 0 & 0 & 0 & 0 & 0 & 0 & 0 & 0 & 0 & 0 & 0 & 0 & 0 & 0 & 0 & 0 \\
\hline $797 \mathrm{~B}-12 \mathrm{H}-6,78-80$ & 109.18 & 0.0824 & 6.0083 & 1.37 & 1.0000 & 0 & 0 & 0 & 0 & $\mathbf{0}$ & 0 & 0 & 0 & $\mathbf{0}$ & 0 & 0 & 0 & 0 & 0 & 0 & 0 & 0 \\
\hline $797 \mathrm{~B}-13 \mathrm{H}-1,78-80$ & 111.18 & 0.0000 & 6.6650 & 0.00 & 1.0000 & 0 & 0 & 0 & 0 & 0 & 0 & 0 & 0 & 0 & 0 & 0 & 0 & 0 & 0 & 0 & 0 & 0 \\
\hline $797 \mathrm{~B}-13 \mathrm{H}-2,78-80$ & 112.68 & 0.0953 & 8.9604 & 1.06 & 1.0000 & 0 & 0 & 0 & 0 & 0 & 0 & 0 & 0 & 0 & 0 & 0 & 0 & 0 & 0 & 0 & 0 & 0 \\
\hline $797 \mathrm{~B}-13 \mathrm{H}-3,78-80$ & 114.18 & 0.2598 & 9.9160 & 2.62 & 1.0000 & 0 & 0 & 0 & 0 & 0 & 0 & 0 & 0 & 0 & 0 & 0 & $\mathbf{0}$ & 0 & 0 & 0 & 0 & 0 \\
\hline $797 \mathrm{~B}-13 \mathrm{H}-4,78-80$ & 115.68 & 0.2888 & 8.5917 & 3.36 & 1.0000 & 0 & 0 & 0 & 0 & 0 & 0 & 0 & 0 & 0 & 0 & 0 & 0 & 0 & 0 & 0 & 0 & 0 \\
\hline 797B-13H-5, 78-80 & 117.18 & 0.1533 & 7.8382 & 1.96 & 1.0000 & 0 & 0 & 0 & 0 & 0 & 0 & 0 & 0 & 0 & 0 & 0 & 0 & 0 & 0 & 0 & 0 & 0 \\
\hline 797B-13H-6, 78-80 & 118.68 & 0,1893 & 8.3226 & 2.27 & 1.0000 & 0 & 0 & 0 & 0 & 0 & 0 & 0 & 0 & 0 & 0 & 0 & 0 & 0 & 0 & 0 & 0 & 0 \\
\hline $797 \mathrm{~B}-14 \mathrm{H}-1,78-80$ & 120.68 & 0.1439 & 7.6248 & 1.89 & 1.0000 & 0 & 0 & 0 & 0 & 0 & 0 & 0 & 0 & 0 & 0 & 0 & 0 & 0 & 0 & 0 & 0 & 0 \\
\hline $797 \mathrm{~B}-14 \mathrm{H}-2,78-80$ & 122.18 & 0.1623 & 6.9918 & 2.32 & 1.0000 & 0 & 0 & 0 & 0 & 0 & 0 & 0 & 0 & 0 & 0 & 0 & 0 & 0 & 0 & 0 & 0 & 0 \\
\hline $797 \mathrm{~B}-14 \mathrm{H}-3,78-80$ & 123.68 & 0.0901 & 6.8596 & 1.31 & 1.0000 & 0 & 0 & 0 & 0 & 0 & 0 & 0 & 0 & 0 & 0 & 0 & 0 & 0 & 0 & 0 & 0 & 0 \\
\hline $797 \mathrm{~B}-14 \mathrm{H}-4,78-80$ & 125.18 & 0.0742 & 6.1573 & 1.21 & 1.0000 & 0 & 0 & 0 & 0 & 0 & 0 & 0 & 0 & 0 & 0 & 0 & $\mathbf{0}$ & 0 & 0 & 0 & 0 & 0 \\
\hline $797 \mathrm{~B}-14 \mathrm{H}-5,78-80$ & 126.68 & 0.2246 & 7.4031 & 3.03 & 1.0000 & 0 & 0 & 0 & 0 & 0 & 0 & 0 & 0 & 0 & 0 & 0 & $\mathbf{0}$ & 0 & 0 & 0 & 0 & 0 \\
\hline $797 \mathrm{~B}-15 \mathrm{H}-1,79-81$ & 130.19 & 0.1008 & 5.2081 & 1.94 & 1.0000 & 0 & 0 & 0 & 0 & 0 & 0 & 0 & 0 & 0 & 0 & 0 & 0 & 0 & 0 & 0 & 0 & 0 \\
\hline $797 \mathrm{~B}-15 \mathrm{H}-2,79-81$ & 131.69 & 0.0359 & 4.6288 & 0.78 & 1.0000 & 0 & 0 & 0 & 0 & 0 & 0 & 0 & 0 & 0 & 0 & 0 & 0 & 0 & 0 & 0 & 0 & 0 \\
\hline $797 \mathrm{~B}-15 \mathrm{H}-3,79-81$ & 133.19 & 0.0230 & 5.3832 & 0.43 & 1.0000 & 0 & 0 & 0 & 0 & 0 & 0 & 0 & 0 & 0 & 0 & 0 & $\mathbf{0}$ & 0 & 0 & 0 & 0 & 0 \\
\hline $797 \mathrm{~B}-15 \mathrm{H}-4,80-82$ & 134.70 & 0.0340 & 5.2789 & 0.64 & 1.0000 & 0 & 0 & 0 & 0 & 0 & 0 & 0 & 0 & 0 & 0 & 0 & $\mathbf{0}$ & 0 & 0 & 0 & 0 & 0 \\
\hline $797 \mathrm{~B}-15 \mathrm{H}-5,79-81$ & 136.19 & 0.0596 & 4.4017 & 1.35 & 1.0000 & 0 & 0 & 0 & 0 & 0 & 0 & 0 & 0 & 0 & 0 & 0 & 0 & 0 & 0 & 0 & 0 & 0 \\
\hline 797B-15H-6, 79-81 & 137.69 & 0.0666 & 5.1132 & 1.30 & 1.0000 & 0 & 0 & 0 & 0 & 0 & 0 & 0 & 0 & 0 & 0 & 0 & 0 & 0 & 0 & 0 & 0 & 0 \\
\hline $797 \mathrm{~B}-16 \mathrm{H}-1,80-82$ & 139.70 & 0.4978 & 6.7217 & 7.41 & 1.0000 & 0 & 0 & 0 & 0 & 0 & 0 & 0 & 0 & 0 & 0 & 0 & 0 & 0 & 0 & 0 & 0 & 0 \\
\hline $797 \mathrm{~B}-16 \mathrm{H}-2,80-82$ & 141.20 & 0.0577 & 8.0013 & 0.72 & 1.0000 & 0 & 0 & 0 & 0 & 0 & 0 & 0 & 0 & 0 & 0 & 0 & 0 & 0 & 0 & 0 & 0 & 0 \\
\hline $797 \mathrm{~B}-16 \mathrm{H}-3,80-82$ & 142.70 & 0.1456 & 8.8090 & 1.65 & 1.0000 & 0 & 0 & 0 & 0 & 0 & 0 & 0 & 0 & 0 & 0 & 0 & 0 & 0 & 0 & 0 & 0 & 0 \\
\hline $797 \mathrm{~B}-16 \mathrm{H}-4,80-82$ & 144.20 & 0.1295 & 8.3125 & 1.56 & 1.0000 & 0 & 0 & 0 & 0 & 0 & 0 & 0 & 0 & 0 & 0 & 0 & 0 & 0 & 0 & 0 & $\mathbf{0}$ & 0 \\
\hline $797 \mathrm{~B}-16 \mathrm{H}-5,77-79$ & 145.67 & 0.0922 & 11.7856 & 0.78 & 1.0000 & 0 & 0 & 0 & 0 & 0 & 0 & 0 & 0 & 0 & 0 & 0 & 0 & 0 & 0 & 0 & 0 & 0 \\
\hline $797 \mathrm{~B}-16 \mathrm{H}-6,79-81$ & 147.19 & 0.1005 & 8.1014 & 1.24 & 1.0000 & 0 & 0 & 0 & 0 & 0 & 0 & 0 & 0 & 0 & 0 & 0 & 0 & 0 & 0 & 0 & 0 & 0 \\
\hline $797 \mathrm{~B}-17 \mathrm{H}-1,79-81$ & 149.19 & 0.0935 & 7.4331 & 1.26 & 1.0000 & 0 & 0 & 0 & 0 & 0 & 0 & 0 & 0 & 0 & 0 & 0 & 0 & 0 & 0 & 0 & 0 & 0 \\
\hline $797 \mathrm{~B}-17 \mathrm{H}-3,40-42$ & 150.60 & 0.0654 & 5.8169 & 1.12 & 1.0000 & 0 & 0 & 0 & 0 & 0 & 0 & 0 & 0 & 0 & 0 & 0 & 0 & 0 & 0 & 0 & 0 & 0 \\
\hline $797 \mathrm{~B}-17 \mathrm{H}-4,79-81$ & 151.56 & 0.0336 & 4.3532 & 0.77 & 1.0000 & 0 & 0 & 0 & 0 & 0 & 0 & 0 & 0 & 0 & 0 & 0 & 0 & 0 & 0 & 0 & 0 & 0 \\
\hline $797 \mathrm{~B}-17 \mathrm{H}-5,79-81$ & 153.06 & 0.1496 & 7.3517 & 2.03 & 1.0000 & 0 & 0 & 0 & 0 & 0 & 0 & 0 & 0 & 0 & 0 & $\mathbf{0}$ & 0 & 0 & 0 & 0 & 0 & 0 \\
\hline $797 \mathrm{~B}-17 \mathrm{H}-6,79-81$ & 154.56 & 0.0345 & 6.1962 & 0.56 & 1.0000 & $\mathbf{0}$ & 0 & 0 & 0 & 0 & 0 & 0 & 0 & 0 & 0 & 0 & 0 & 0 & 0 & 0 & 0 & 0 \\
\hline $797 \mathrm{~B}-17 \mathrm{H}-7,79-81$ & 156.06 & 0.1530 & 7.6429 & 2.00 & 1.0000 & 0 & 0 & 0 & 0 & 0 & 0 & 0 & 0 & 0 & 0 & 0 & 0 & 0 & 0 & 0 & 0 & 0 \\
\hline $797 \mathrm{~B}-18 \mathrm{H}-1,80-82$ & 158.70 & 0.0863 & 7.3654 & 1.17 & 1.0000 & 0 & 0 & 0 & 0 & 0 & 0 & 0 & 0 & 0 & 0 & 0 & 0 & 0 & 0 & 0 & 0 & 0 \\
\hline $797 \mathrm{~B}-18 \mathrm{H}-2,80-82$ & 160.20 & 0.1611 & 6.6940 & 2.41 & 1.0000 & 0 & 0 & 0 & $\mathbf{0}$ & 0 & 0 & 0 & 0 & 0 & 0 & 0 & 0 & 0 & 0 & 0 & 0 & 0 \\
\hline $797 \mathrm{~B}-18 \mathrm{H}-3,70-72$ & 161.60 & 0.2310 & 7.46662 & 3.09 & 1.0000 & 0 & 0 & 0 & 0 & 0 & 0 & 0 & 0 & 0 & 0 & 0 & 0 & 0 & 0 & 0 & 0 & 0 \\
\hline $797 \mathrm{~B}-18 \mathrm{H}-4,80-82$ & 163.20 & 0.2506 & 8.6653 & 2.89 & 1.0000 & 0 & 0 & 0 & 0 & 0 & 0 & 0 & 0 & 0 & 0 & 0 & 0 & 0 & 0 & 0 & 0 & 0 \\
\hline $797 \mathrm{~B}-18 \mathrm{H}-5,80-82$ & 164.70 & 0.1172 & 7.3798 & 1.59 & 1.0000 & 0 & 0 & 0 & 0 & 0 & 0 & 0 & 0 & 0 & 0 & 0 & 0 & 0 & 0 & 0 & 0 & 0 \\
\hline $797 \mathrm{~B}-18 \mathrm{H}-6,60-62$ & 166.00 & 0.1360 & 5.5749 & 2.44 & 1.0000 & 0 & 0 & $\mathbf{0}$ & 0 & 0 & 0 & 0 & 0 & 0 & 0 & 0 & 0 & 0 & 0 & 0 & 0 & 0 \\
\hline $797 \mathrm{~B}-19 \mathrm{H}-1,79-81$ & 167.19 & 0.3318 & 6.5383 & 5.07 & 1.0000 & 0 & 0 & 0 & 0 & 0 & 0 & 0 & 0 & $\mathbf{0}$ & 0 & 0 & 0 & 0 & 0 & 0 & 0 & 0 \\
\hline 797B-19H-2, 79-81 & 168.69 & 0.0802 & 7.6172 & 1.05 & 1.0000 & 0 & 0 & 0 & 0 & 0 & 0 & 0 & 0 & 0 & 0 & 0 & 0 & 0 & 0 & 0 & 0 & 0 \\
\hline $797 \mathrm{~B}-19 \mathrm{H}-3,79-81$ & 170.19 & 0.1204 & 6.9454 & 1.73 & 1.0000 & 0 & 0 & 0 & 0 & 0 & 0 & 0 & 0 & 0 & 0 & 0 & 0 & 0 & 0 & 0 & 0 & 0 \\
\hline $797 \mathrm{~B}-19 \mathrm{H}-4,79-81$ & 171.69 & 0.1743 & 7.1394 & 2.44 & 1.0000 & 0 & 0 & 0 & 0 & 0 & 0 & 0 & 0 & 0 & 0 & 0 & 0 & 0 & 0 & 0 & 0 & 0 \\
\hline $797 \mathrm{~B}-19 \mathrm{H}-5,79-81$ & 173.19 & 0.1452 & 8.0460 & 1.80 & 1.0000 & 0 & 0 & $\mathbf{0}$ & 0 & 0 & 0 & 0 & 0 & 0 & 0 & 0 & 0 & 0 & 0 & 0 & 0 & 0 \\
\hline 797B-19H-6, 79-81 & 174.69 & 0.1300 & 8.3638 & 1.55 & 1.0000 & 0 & 0 & 0 & 0 & 0 & 0 & 0 & 0 & 0 & 0 & 0 & 0 & 0 & 0 & 0 & 0 & 0 \\
\hline
\end{tabular}

Sand weight $\quad$ weight of $>63-\mu \mathrm{m}$ fraction

Sed weight weight of the total, dry sediment sample

Sand $\%$ percent by weight of the $>63-\mu \mathrm{m}$ fraction

Split fraction of the sample counted for foraminifers

Pla num number of planktonic foraminifer tests per gram of sediment

Ben num number of benthonic foraminiferal tests per gram of sediment

ratio: $100 \times$ (planktonic V(planktonic + benthic foraminifers)

Coil Rat ratio: $100 \times$ (sinistral Nq. pachyderma/all Nq. pachyderma)

\#Pla

mintank or plaminifers counted

PACL \% relative frequency of sinistral Neogloboquadrina pachvderma in planktonic foraminiferal assemblage

PACR \% relative frequency of dextral Neogloboquadrina pachyderma in planktonic foraminifer assemblage

BUL \% relative frequency of Globigerina bulloides in planktonic foraminifer assemblage

QU1 \% relative frequency of Globigerina umbilicata in planktonic foraminifer assemblage

$\begin{array}{ll}\text { QU1 \% } & \text { relative frequency of Globigerina quinqueloba in planktonic foraminifer assemblage } \\ \text { GLU \% } & \text { relative frequency of Globigerinita glutinata in planktonic foraminifer assemblage }\end{array}$

relative frequency of Globigerinita glutinata in planktonic foraminifer assemblage

relative frequency of Globigerinita minuta in planktonic foraminifer assemblage

$\begin{array}{ll}\text { UVU \% } & \text { relative frequency of Globigerinita uvula in planktonic foraminifer assemblage } \\ \text { PAR } \% & \text { relative frequency of Tenuitella parkerae in planktonic foraminifer assemblage }\end{array}$

HUM \% relative frequency of Turborotalita humilis in planktonic foraminifer as semblage

IND \% relative frequency of indeterminate tests in planktonic foraminifer assemblage 
Table 9. Constituents of sand-size fraction.

\begin{tabular}{|c|c|c|c|c|c|c|}
\hline Sample 127. & $\begin{array}{l}\text { Depth } \\
\text { (mbsf) }\end{array}$ & $\begin{array}{l}\text { Sand } \\
(\%)\end{array}$ & $\begin{array}{c}\text { Plank. } \\
\text { foram. } \\
\text { number } \\
(\# / g)\end{array}$ & Major constituent & Minor constituent-1 & Minor constituent-2 \\
\hline $794 \mathrm{~A}-2 \mathrm{H}-6,17-20 \mathrm{~cm}$ & 14.47 & 3,10 & 6416 & Foraminifers & Minerals & \\
\hline $794 \mathrm{~A}-2 \mathrm{H}-6,84-87$ & 15.14 & 0.57 & 42 & Minerals & Lithic fragments & \\
\hline $794 \mathrm{~A}-2 \mathrm{H}-6,96-98$ & 15.26 & 7.59 & 27770 & Foraminifers & Anhydrite & \\
\hline $794 \mathrm{~A}-3 \mathrm{H}-1,94-99$ & 17.24 & 5.88 & 13836 & Foraminifers & Gypsum & \\
\hline $794 \mathrm{~A}-3 \mathrm{H}-1,117-120$ & 17.47 & 9.44 & 22366 & Foraminifers & & \\
\hline $794 \mathrm{~A}-4 \mathrm{H}-6,95-100$ & 34.25 & & 89 & Gypsum & Anhydrite & Foraminifers \\
\hline $794 \mathrm{~A}-4 \mathrm{H}-7,40-42$ & 35.20 & 10.43 & 11276 & Foraminifers & Pyrite & Anhydrite \\
\hline 797B-1H-1, 134-136 & 1.34 & 1.22 & 73 & Pyrite & Minerals & \\
\hline $797 \mathrm{~B}-1 \mathrm{H}-2,13-15$ & 1.63 & 1.64 & 1174 & Foraminifers & Minerals & Radiolarians \\
\hline $797 \mathrm{~B}-2 \mathrm{H}-1,102-104$ & 6.92 & 4.13 & 877 & Foraminifers & Pyrite & Radiolarians \\
\hline $797 \mathrm{~B}-2 \mathrm{H}-6,134-136$ & 14.74 & 5.35 & 155 & Pumice & Pyrite & \\
\hline 797B-3H-6, 135-137 & 24.25 & 3.86 & 12585 & Foraminifers & & \\
\hline $797 \mathrm{~B}-3 \mathrm{H}-7,14-16$ & 24.54 & 2.14 & 8428 & Foraminifers & Spicules & Radiolarians and Diatoms \\
\hline $797 \mathrm{~B}-4 \mathrm{H}-3,44-46$ & 28.34 & 4.60 & 50 & Pumice & & \\
\hline 797B-4H-3, 77-79 & 28.67 & 1.15 & 54 & Anhydrite & Foraminifers & \\
\hline $797 \mathrm{~B}-4 \mathrm{H}-3,102-104$ & 28.92 & 2.57 & 3344 & Foraminifers & & \\
\hline $797 \mathrm{~B}-4 \mathrm{H}-3,135-137$ & 29.25 & 25.01 & 1943 & Pyrite & Foraminifers & \\
\hline $797 \mathrm{~B}-4 \mathrm{H}-4,102-104 *$ & 30.42 & 3.13 & 1864 & Foraminifers & Pyrite & \\
\hline $797 \mathrm{~B}-4 \mathrm{H}-4,135-137$ & 30.75 & 14.57 & 32407 & Foraminifers & & \\
\hline $797 \mathrm{~B}-4 \mathrm{H}-5,43-45$ & 31.33 & 6.85 & 8304 & Foraminifers & Pyrite & \\
\hline $797 \mathrm{~B}-4 \mathrm{H}-6,135-137$ & 33.75 & 0.94 & 2837 & Foraminifers & & \\
\hline $797 \mathrm{~B}-5 \mathrm{H}-1,132-134$ & 35.72 & 1.70 & 881 & Foraminifers & Pyrite & Minerals \\
\hline $797 \mathrm{~B}-5 \mathrm{H}-2,103-105^{*}$ & 36.93 & 1.33 & 113 & Minerals & Pyrite & Foraminifers \\
\hline $797 \mathrm{~B}-5 \mathrm{H}-4,74-76$ & 39.64 & 0.80 & 61 & Minerals & & \\
\hline 797B-5H-4, 78-80 & 39.68 & 14.59 & 17073 & Foraminifers & Anhydrite & \\
\hline 797B-5H-5, I1-13* & 40.51 & 0.78 & 544 & Minerals & Foraminifers & Spicules \\
\hline 797B-5H-5, 43-45 & 40.83 & 1.16 & 51 & Ash & Radiolarians and Diatoms & Foraminifers \\
\hline 797B-5H-5, 131-133 & 41.71 & 1.46 & 2689 & Foraminifers & & \\
\hline 797B-5H-6, 102-104* & 42.92 & 8.03 & 10048 & Foraminifers & Spicules & Organics \\
\hline 797B-5H-7, 43-45 & 43.83 & 2.02 & 1429 & Foraminifers & Pyrite & Minerals \\
\hline $797 \mathrm{~B}-6 \mathrm{H}-1,132-134$ & 45.22 & 4.60 & 110 & Spicules & Pyrite & \\
\hline 797B-6H-2, 74-76 & 46.14 & 1.00 & 609 & Foraminifers & Pyrite & Minerals \\
\hline 797B-6H-2, 78-80 & 46.18 & 2.02 & 2107 & Foraminifers & Pyrite & Minerals \\
\hline 797B-6H-2, 102-104 & 46.42 & 1.95 & 608 & Foraminifers & & \\
\hline 797B-6H-3, 74-76 & 47.64 & 8.93 & 3961 & Foraminifers & Pyrite & \\
\hline 797B-6H-3, 78-80 & 47.68 & 0.96 & 687 & Foraminifers & Radiolarians and Diatoms & Minerals \\
\hline 797B-6H-3, 102-104 & 47.92 & 3.44 & 5197 & Foraminifers & $\begin{array}{l}\text { Pyrite } \\
\end{array}$ & Minerals \\
\hline 797B-10H-2, 78-80 & 84.18 & 0.39 & 361 & Foraminifers & Pyrite & Minerals \\
\hline
\end{tabular}

*thin, foraminifer-rich layer

5. Deep waters of the nascent and mature backarc basin were poorly oxygenated throughout most of the Miocene sequences. Oxygenation improved in the uppermost Miocene and continued through the Pliocene.

6. The Quaternary assemblage of benthic foraminifers appears shallower than the basins they were cored from, based on depth ranges of selected foraminifers. The anomalous assemblage was introduced into the basin near the base of the Pleistocene and persists to the present day.

7. Quaternary planktonic assemblages are similar to assemblages known from the latest Pleistocene of the Japan Sea, whereas the Olduvai assemblage is similar to present-day faunas of the transitional North Pacific Ocean.

\section{ACKNOWLEDGMENTS}

Yasumochi Matoba and Michio Kato deserve special thanks for their help and advice concerning identification of benthic foraminifers and assistance in collection of comparative material from the Oga Peninsula. Ryuji Tada generously provided key samples at Sites 794 and 797. Jim Ingle and Tara Kheradyar helped in many useful discussions of the data as did Itaru Koizumi, Atiur Rahman, Lisa White, Joanne Alexandrovich, and all my colleagues on board Leg 127. The reviews by Peter Thompson and Kristin McDougall are greatly appreciated as are the editorial comments of John Barron. This research was supported by JOI.

\section{REFERENCES}

Asano, K., 1950-1951. Illustrated Catalogue of Japanese Tertiary Smaller Foraminifera (Parts 1-15): Tokyo (Hosokawa Printing Co.).

Asano, K., Ingle, J. C., Jr., and Takayanagi, Y., 1969. Neogene planktonic foraminiferal sequence in northern Honshu. In Brönniman, R. P., and Renz, H. (Eds.), Proc. 1st Int. Conf. Planktonic Microfossils: Leiden (E. J. Brill), 1:14-25.

Berggren, W. A., Kent, D. V., and Van Couvering, J. A., 1985. The Neogene: Part 2. Neogene geochronology and chronostratigraphy. In Snelling, N. J. (Ed.), The Chronology of the Geological Record. Geol. Soc. London Mem., 10:211-260.

Burckle, L. H., and Todd, A., 1976. Correlation of Late Neogene sections of the Noto and Oga peninsulas, Japan. In Takayanagi, U., and Saito, T. (Eds.), Progress in Micropaleontology: New York (Micropaleontology Press), 20-26.

Chinzei, K., 1986. Opening of the Japan Sea and marine biogeography during the Miocene. J. Geomagn. Geoelectr., 38:487-494.

Coulbourn, W. T., Parker, F. L., and Berger, W. H., 1980. Faunal and solution patterns of planktonic foraminifera in surface sediments of the North Pacific. Mar. Micropaleontol., 5:329-399.

Culver, S. J., and Buzas, M. A., 1986. Distribution of Recent benthic foraminifera off the North American Pacific coast from California to Baja. Smithsonian Contrib. Mar. Sci., 28:1-634.

Guber, A. L., and Merrill, S., III, 1983. Paleobathymetric significance of the foraminifera from the Hokuroku District. Econ. Geol. Monogr., 5:55-70.

Ichikura, M., and Ujiié, H., 1976. Lithology and planktonic foraminifera of the Sea of Japan piston cores. Bull. Nat. Sci. Mus., Ser. C (Geol.), 2:151-178.

Ingle, J. C., Jr., 1975. Pleistocene and Pliocene foraminifera from the Sea of Japan, Leg 31, Deep Sea Drilling Project. In Karig, D. E., Ingle, J. C., Jr., et al., Init. Repts. DSDP, 31: Washington (U.S. Govt. Printing Office), 693-701. 
Ingle, J. C., Jr., and Keller, G., 1980. Benthic foraminiferal biofacies of the eastern Pacific margin between $40^{\circ} \mathrm{S}$ and $32^{\circ} \mathrm{N}$. In Quaternary Depositional Environments of the Pacific Coast. Paleogeogr. Symp. 4, Pacific Sect., Soc. Econ. Paleontol. Mineral., 341-355.

Ingle, J. C., Jr., Keller, G., and Kolpack, R. L., 1980. Benthic foraminiferal biofacies, sediments and water masses of the southern Peru-Chile Trench area, southeastern Pacific Ocean. Micropaleontology, 26:113-150.

Kato, M., 1984. Foraminifera: analysis of the piston core, KH-79-3, C-3. Gekkan Chikyu, 6:529-537.

1989. Benthic foraminifers from the Japan Sea piston cores. In Takayanagi, Y., and Ishizaki, K. (Eds.), Collected Papers on Foraminifera from the Japanese Islands: Sendai, Japan, 111-116.

Keller, G., 1980. Benthic foraminifers and paleobathymetry of the Japan Trench area, Leg 57, Deep Sea Drilling Project. In von Huene, R., Nasu, N., et al., Init. Repts. DSDP, 56, 57 (Pt. 2): Washington (U.S. Govt. Printing Office), 835-865.

Kennett, J. P., and Srinivasan, M. S., 1983. Neogene Planktonic Foraminifera: Stroudsburg, PA (Hutchinson Ross).

Kurihara, K., 1976. Correlation of Neogene formations between the Japan Sea and the Pacific coast regions of Japan, by benthonic foraminifera. Rev. Esp. Micropaleontol., 9:307-315.

Lagoe, M. B., and Thompson, P. R., 1988. Chronostratigraphic significance of Late Cenozoic planktonic foraminifera from the Ventura Basin, California: potential for improving tectonic and depositional interpretation. J. Foraminiferal. Res., 18:250-266.

Maiya, S., Saito, T., and Sato, T., 1976. Late Cenozoic planktonic foraminiferal biostratigraphy of northwest Pacific sedimentary sequences. In Takayanagi, Y., and Saito, T. (Eds.), Progress in Micropaleontology: New York (Micropaleontology Press), 395-422.

Matoba, Y., 1977. Recent foraminiferal assemblages off Sendai, northeastern Japan. In Schafer, C. T., and Pelletier, B. R. (Eds.), First Int. Symp. on Benthonic Foraminifera of Continental Margins, Maritime Sediments, 205-220.

, 1984. Paleoenvironment of the Sea of Japan. In Oertli, J. J. (Ed.), Benthos '83: Proc. 2nd Int. Symp. Benthic Foraminifera (Pau, April 1983), 409-414.

Matoba, Y., Tomizawa, A., Maruyama, T., Shiraishi, T., Aita, Y., and Okamoto, K., 1990. Neogene and Quaternary sedimentary sequences in the Oga Peninsula. Benthos '90, Proc. 4th Int. Symp. Benthic Foraminifera, Sendai, Japan, Guidebook for Field Trip No. 2: B1-B62 with 12 plates appended.
Matsunaga, T., 1963. Benthonic smaller foraminifera from the oil fields of Northern Japan. Sci. Rep. Tohoku Univ., Ser. 2, 35:67-122 and 52 plates.

Oda, M., 1986. Some aspects and problems concerned with microfossil biochronology for the Neogene in Central and Northeast Honshu, Japan. In Nakagawa, H., Kotaka, T., and Takayanagi, Y. (Eds.), The Essays on Geology of Professor N. Kitamura: Sendai (Sasaki Print Co.), 195-211.

Reynolds, L., and Thunell, R. C., 1986. Seasonal production and morphologic variation of Neogloboquadrina pachyderma (Ehrenberg) in the northeast Pacific. Micropaleontology, 32:1-18.

Saito, T., 1963. Miocene planktonic foraminifera from Honshu, Japan. Sci. Rep. Tohoko Univ., Ser. 2, 35:123-209.

Saito, T., and Maiya, S., 1973. Planktonic foraminifera of the Nishikurosawa Formation, northeast Honshu, Japan. Trans. Proc. Paleontol. Soc. Jpn., 91:113-125.

Sautter, L. R., and Thunell, R. C., 1989. Seasonal succession of planktonic foraminifera: results from a four-year time-series sediment trap experiment in the northeast Pacific. J. Foraminiferal Res., 19:253-267.

1991. Planktonic foraminiferal response to upwelling and seasonal hydrographic conditions: sediment trap results from San Pedro Basin, Southern Califormia Bight. J. Foraminiferal Res., 21:347-363.

Tamaki, K., Pisciotto, K., Allan, J., et al., 1990. Proc. ODP, Init. Repts., 127: College Station, TX (Ocean Drilling Program).

Thompson, P. R., 1980. Foraminifers from Deep Sea Drilling Project Sites 434, 435, and 436, Japan Trench. In von Huene, R., Nasu, N., et al., Init. Repts. DSDP, 56, 57 (Pt. 2): Washington (U.S. Govt. Printing Office), 775-808.

Tsuchi, R., and IGCP-114 National Working Group of Japan, 1984. Neogene chronostratigraphy and bio-events in the Japanese Islands. Paleogeogr., Paleoclimatol., Paleoecol., 46:38-51.

Ujiié, H., and Ichikura, M., 1973. Holocene to uppermost Pleistocene planktonic foraminifers in a piston core from off San'in District, Sea of Japan. Trans. Proc. Palaeontol. Soc. Jpn., 91:137-150.

Date of initial receipt: 18 March 1991

Date of acceptance: 5 December 1991

Ms 127/128B-124 
Site 794

Site 797

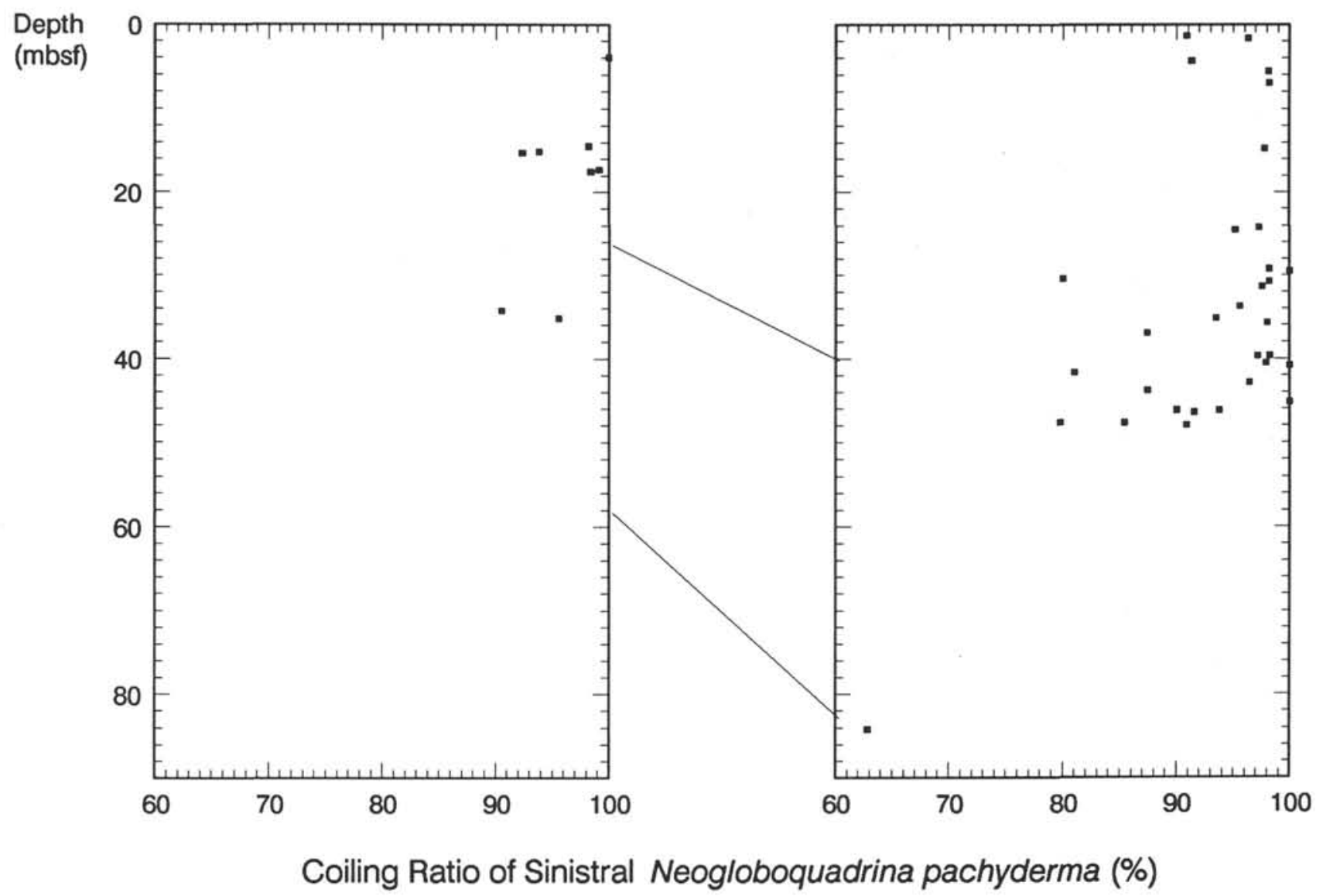

Figure 2. The coiling ratio of Neogloboquadrina pachyderma (sinistral forms/total number of Neogloboquadrina pachyderma in sample) plotted with depth at Holes 794A and 797B. All samples that plot on the $y$ axis are barren or have fewer than 20 specimens of the species. The two lines connecting the graphs denote the Brunhes/Matuyama boundary and the top of the Olduvai. 
Table 10. Coiling ratio of Neogloboquadrina pachyderma with number of tests of sinistral and dextral forms counted to calculate the ratio.

\begin{tabular}{|c|c|c|c|c|}
\hline Sample 127- & $\begin{array}{l}\text { Depth } \\
\text { (mbsf) }\end{array}$ & PacL & PacR & $\begin{array}{r}\text { Coil } \\
(\%)\end{array}$ \\
\hline $794 \mathrm{~A}-1 \mathrm{H}-3,93-98 \mathrm{~cm}$ & 3.93 & 41 & 0 & 100 \\
\hline $794 \mathrm{~A}-2 \mathrm{H}-6,17-20$ & 14.47 & 27 & 5 & 98 \\
\hline $794 \mathrm{~A}-2 \mathrm{H}-6,84-87$ & 15.14 & 16 & 1 & 94 \\
\hline $794 \mathrm{~A}-2 \mathrm{H}-6,96-98$ & 15.26 & 156 & 13 & 92 \\
\hline 794A-3H-1, 94-99 & 17.24 & 232 & 2 & 99 \\
\hline $794 \mathrm{~A}-3 \mathrm{H}-1,117-120$ & 17.47 & 371 & 6 & 98 \\
\hline $794 \mathrm{~A}-4 \mathrm{H}-6,95-100$ & 34.25 & 200 & 21 & 90 \\
\hline $794 \mathrm{~A}-4 \mathrm{H}-7,40-42$ & 35.20 & 301 & 14 & 96 \\
\hline $797 \mathrm{~A}-1 \mathrm{H}-1,76-78$ & 4.16 & 27 & 0 & 100 \\
\hline $797 \mathrm{~A}-1 \mathrm{H}-2,76-78$ & 5.66 & 19 & 3 & 86 \\
\hline 797B-1H-1, 134-136 & 1.34 & 169 & 17 & 91 \\
\hline $797 \mathrm{~B}-1 \mathrm{H}-2,13-15$ & 1.63 & 339 & 13 & 96 \\
\hline 797B-1H-3, 134-136 & 4.34 & 21 & 2 & 91 \\
\hline 797B-1H-4, 104-106 & 5.54 & 105 & 2 & 98 \\
\hline $797 \mathrm{~B}-2 \mathrm{H}-1,102-104$ & 6.92 & 216 & 4 & 98 \\
\hline 797B-2H-6, $134-136$ & 14.74 & 264 & 6 & 98 \\
\hline 797B-3H-6, 135-137 & 24.25 & 526 & 15 & 97 \\
\hline $797 \mathrm{~B}-3 \mathrm{H}-7,14-16$ & 24.54 & 140 & 7 & 95 \\
\hline $797 \mathrm{~B}-4 \mathrm{H}-3,135-137$ & 29.25 & 216 & 4 & 98 \\
\hline $797 \mathrm{~B}-4 \mathrm{H}-4,13-15$ & 29.53 & 35 & 0 & 100 \\
\hline $797 \mathrm{~B}-4 \mathrm{H}-4,102-104$ & 30.42 & 19 & 3 & 80 \\
\hline $797 \mathrm{~B}-4 \mathrm{H}-4,135-137$ & 30.75 & 320 & 6 & 98 \\
\hline $797 \mathrm{~B}-4 \mathrm{H}-5,43-45$ & 31.33 & 240 & 6 & 98 \\
\hline 797B-4H-6, 135-137 & 33.75 & 487 & 22 & 96 \\
\hline 797B-5H-1, 78-80 & 35.18 & 43 & 3 & 93 \\
\hline 797B-5H-1, 132-134 & 35.72 & 148 & 3 & 98 \\
\hline 797B-5H-2, 103-105 & 36.93 & 146 & 21 & 87 \\
\hline $797 \mathrm{~B}-5 \mathrm{H}-4,74-76$ & 39.64 & 225 & 4 & 98 \\
\hline 797B-5H-4, 78-80 & 39.68 & 208 & 6 & 97 \\
\hline 797B-5H-5, 11-13 & 40.51 & 648 & 14 & 98 \\
\hline 797B-5H-5, 43-45 & 40.83 & 141 & 0 & 100 \\
\hline $797 \mathrm{~B}-5 \mathrm{H}-5,131-133$ & 41.71 & 238 & 33 & 81 \\
\hline 797B-5H-6, 102-104 & 42.92 & 268 & 9 & 96 \\
\hline 797B-5H-7, 43-45 & 43.83 & 181 & 26 & 87 \\
\hline 797B-6H-1, 132-134 & 45.22 & 33 & 0 & 100 \\
\hline $797 \mathrm{~B}-6 \mathrm{H}-2,74-76$ & 46.14 & 226 & 25 & 90 \\
\hline 797B-6H-2, 78-80 & 46.18 & 330 & 22 & 94 \\
\hline 797B-6H-2, 102-104 & 46.42 & 292 & 27 & 92 \\
\hline 797B-6H-3, 74-76 & 47.64 & 221 & 56 & 80 \\
\hline $797 \mathrm{~B}-6 \mathrm{H}-3,78-80$ & 47.68 & 281 & 48 & 85 \\
\hline 797B-6H-3, 102-104 & 47.92 & 308 & 31 & 91 \\
\hline 797B-10H-2, 78-80 & 84.18 & 89 & 0 & 63 \\
\hline
\end{tabular}

PacL-number of tests of sinistral Neogloboquadrina pachyderma counted

PacR-number of tests of dextral Neogloboquadrina pachyderma counted

Coil-ratio of sinistral to total number of specimens of Neogloboquadrina pachyderma 
EUCLIDEAN DISTANCE COEFFICIENT

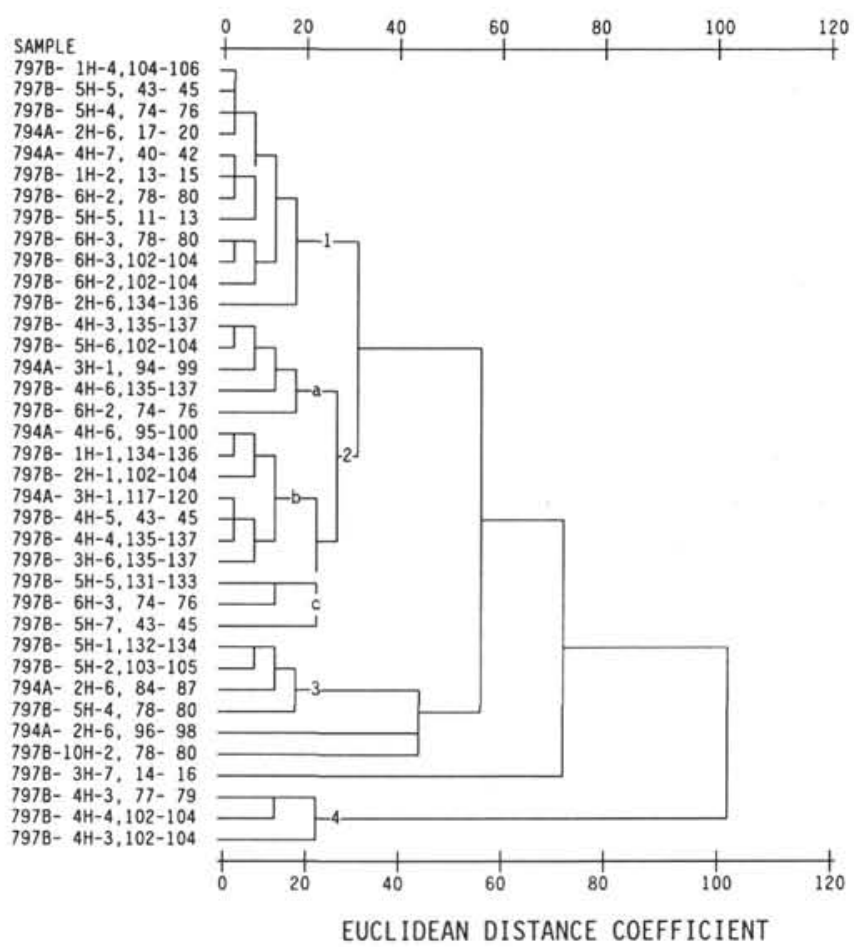

Figure 3. Dendrogram of Q-mode analysis of all samples with 100 or more specimens of planktonic foraminifers. 
Table 11. Relative frequencies of planktonic foraminifers in samples arranged by cluster.

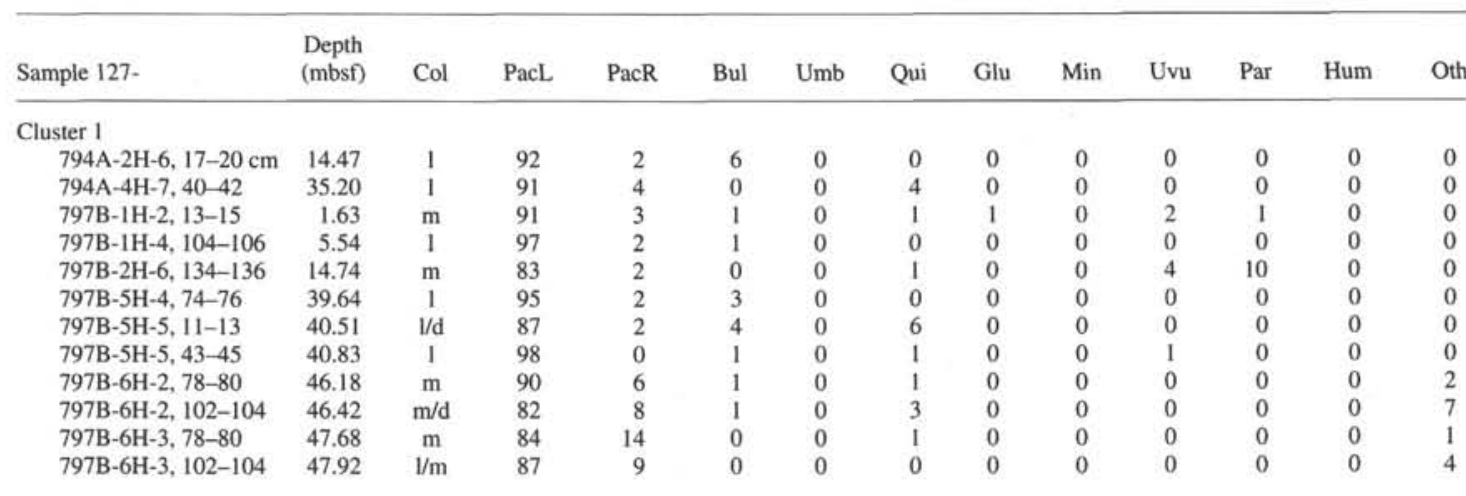

Cluster 2

Subgroup a

794A-3H-1, 94-99 17.24

797B-4H-3, 135-137 29.25

$797 \mathrm{~B}-4 \mathrm{H}-6,135-137 \quad 33.7$

797B-5H-6, 102-104 42.92

$797 \mathrm{~B}-6 \mathrm{H}-2,74-76 \quad 46.14$

$\begin{array}{ll}1 & 67\end{array}$

d 74

m 82

m 71

Subgroup b

794A-3H-1, 117-120 $\quad 17.47$

$794 \mathrm{~A}-4 \mathrm{H}-6,95-100 \quad 34.25$

797B-1H-1, 134-136 1.34

797B-2H-1, 102-104 6.92

797B-3H-6, 135-137 24.25

797B-4H-4, 135-137 30.75

797B-4H-5, 43-45 31.33

Subgroup c

797B-5H-5, 131-133 41.7

797B-5H-7, 43-45 43.83

$797 \mathrm{~B}-6 \mathrm{H}-3,74-76 \quad 47.64$

Cluster 3

794A-2H-6, 84-87 15

797B-5H-1, 132-134 35.72

$797 \mathrm{~B}-5 \mathrm{H}-2,103-105 \quad 36.93$

797B-5H-4, 78-80 39.68

$1 \quad 77$

d 65

m 69

d $\quad 71$

m 77

$\begin{array}{cccc}\mathrm{m} & 72 & 1 & 21 \\ \mathrm{I} & 75 & 2 & 20\end{array}$

$\begin{array}{lll}1 & 70 \quad 16\end{array}$

1/d $\quad 56$

138

$1 / \mathrm{m} \quad 46$

$\mathrm{l} / \mathrm{m} \quad 43$

Cluster 4

797B-4H-3, 77-79

$797 \mathrm{~B}-4 \mathrm{H}-3,102-104$

$797 \mathrm{~B}-4 \mathrm{H}-4,102-104 \quad 30.42$

Unclusterd samples

$\begin{array}{cccccrrrrrrr}\mathrm{d} & 1 & 0 & 99 & 0 & 0 & 0 & 0 & 0 & 0 & 0 & 0 \\ \mathrm{I} & 1 & 1 & 80 & 0 & 16 & 0 & 0 & 1 & 1 & 0 & 0 \\ \mathrm{l} / \mathrm{d} & 6 & 1 & 87 & 2 & 3 & 0 & 0 & 0 & 0 & 0 & 0\end{array}$

\footnotetext{
794A-2H-6, 96-98

797B-10H-2, 78-80

\begin{tabular}{|c|c|c|c|c|c|c|c|c|c|c|c|c|}
\hline $794 \mathrm{~A}-2 \mathrm{H}-6,96-98$ & 15.26 & d & 51 & 4 & 13 & 0 & 6 & 0 & 0 & 1 & 10 & 13 \\
\hline $797 \mathrm{~B}-10 \mathrm{H}-2.78-80$ & 84.18 & $\mathrm{~m}$ & 23 & 13 & 27 & 0 & 24 & 0 & 1 & 4 & 7 & 0 \\
\hline
\end{tabular}

PacL sinistral Neogloboquadrina pachyderma

PacR dextral Neogloboquadrina pachyderma

Bul Globigerina bulloides

Umb Globigerina umbilicata

Qui Globigerina quinqueloba

Glu Globigerinita glutinata

Min Globigerinita minuta

Uvu Globigerinita uvula

Par Tenuitella parkerae

Hum Turborotalita humilis

Oth other planktonic foraminiferal species

d dark chocolate brown sediment

m medium brown sediment

$1 \quad$ light putty gray sediment
} 


\section{A. BRUNNER}

Table 12. Correlation of diatom and foraminifer zones.

\section{A. Site 794}

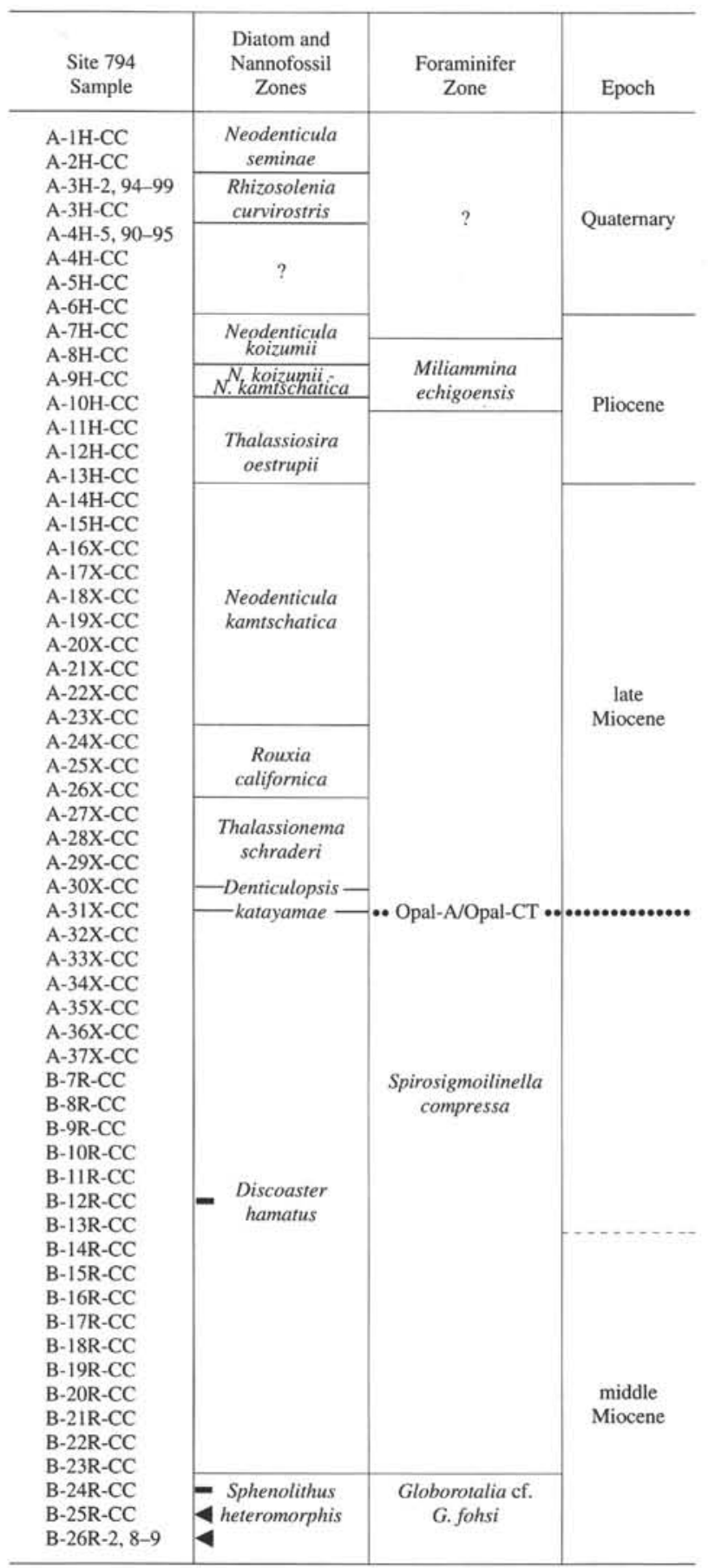

Table 12 (continued).

B. Site 795

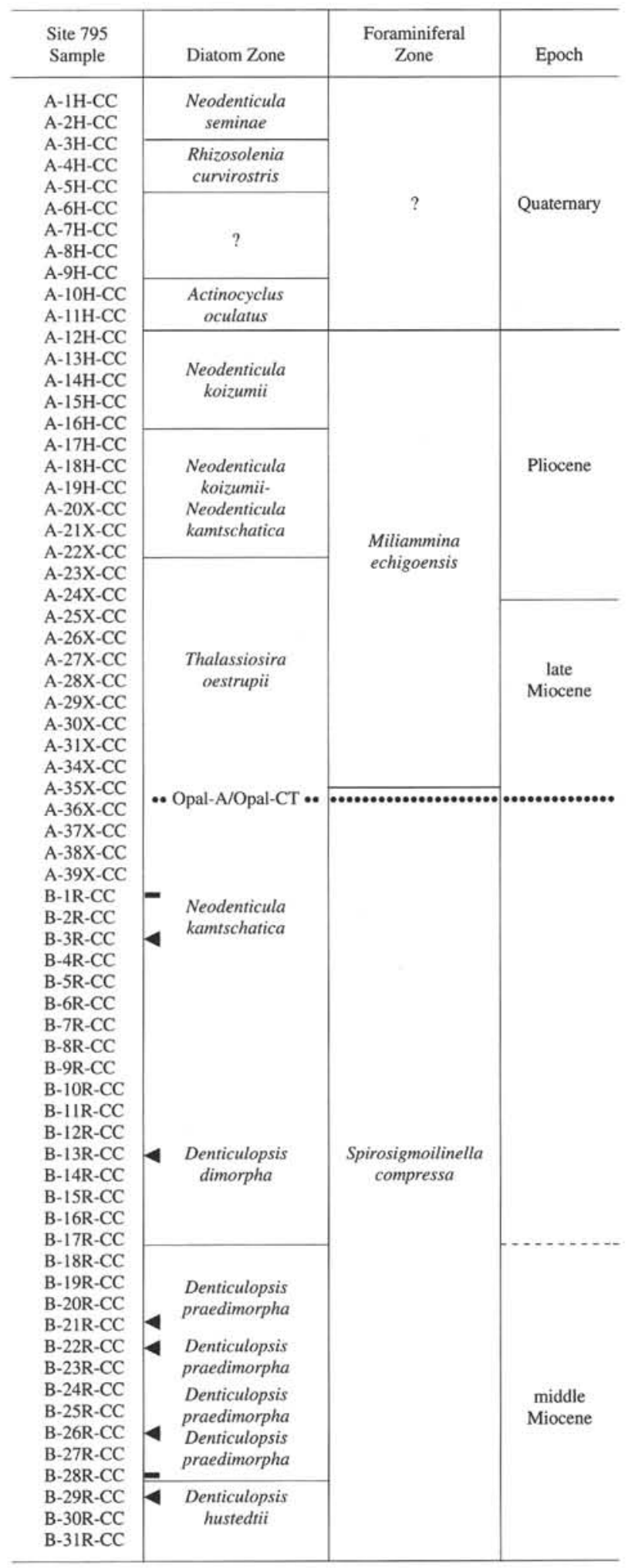


Table 12 (continued).

\section{Site 796}

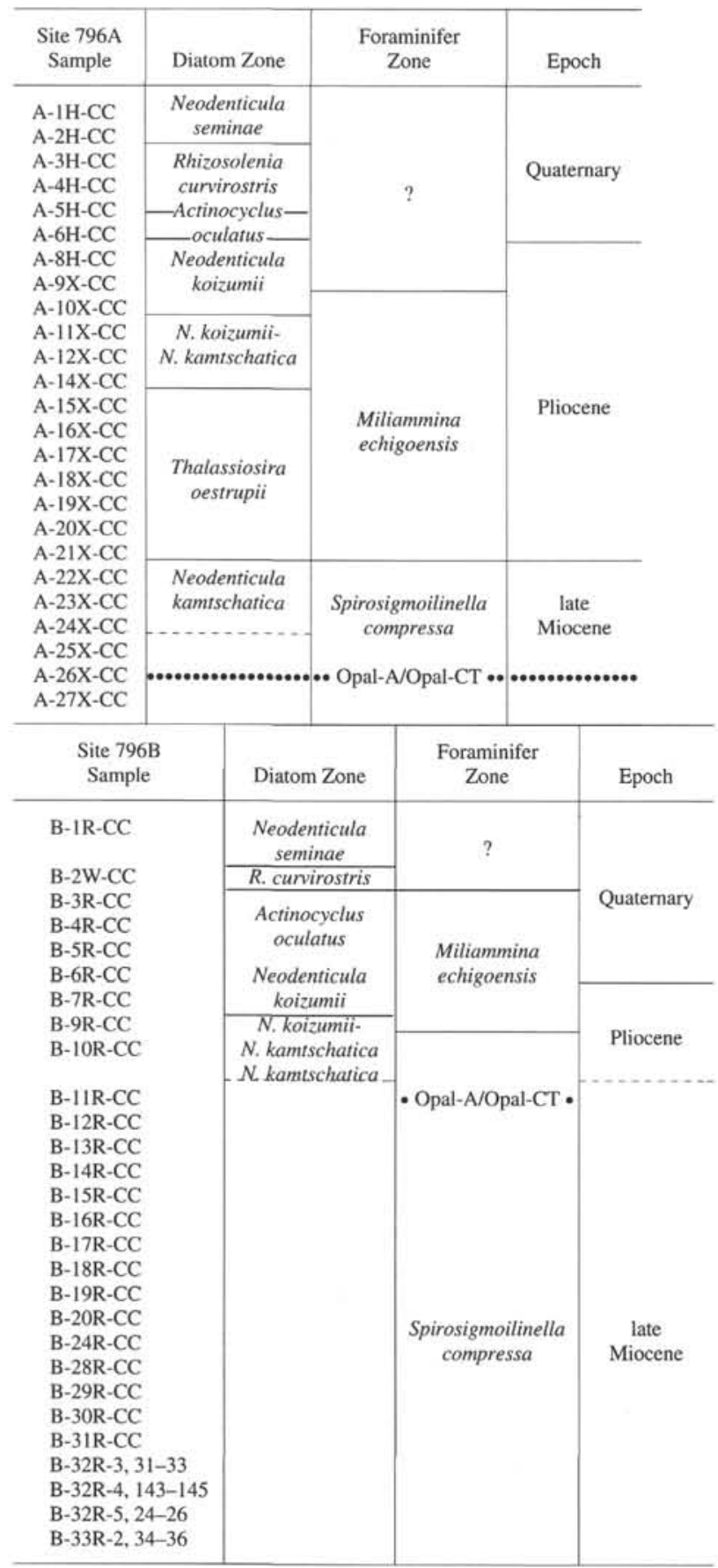

Table 12 (continued).

\section{Site 797}

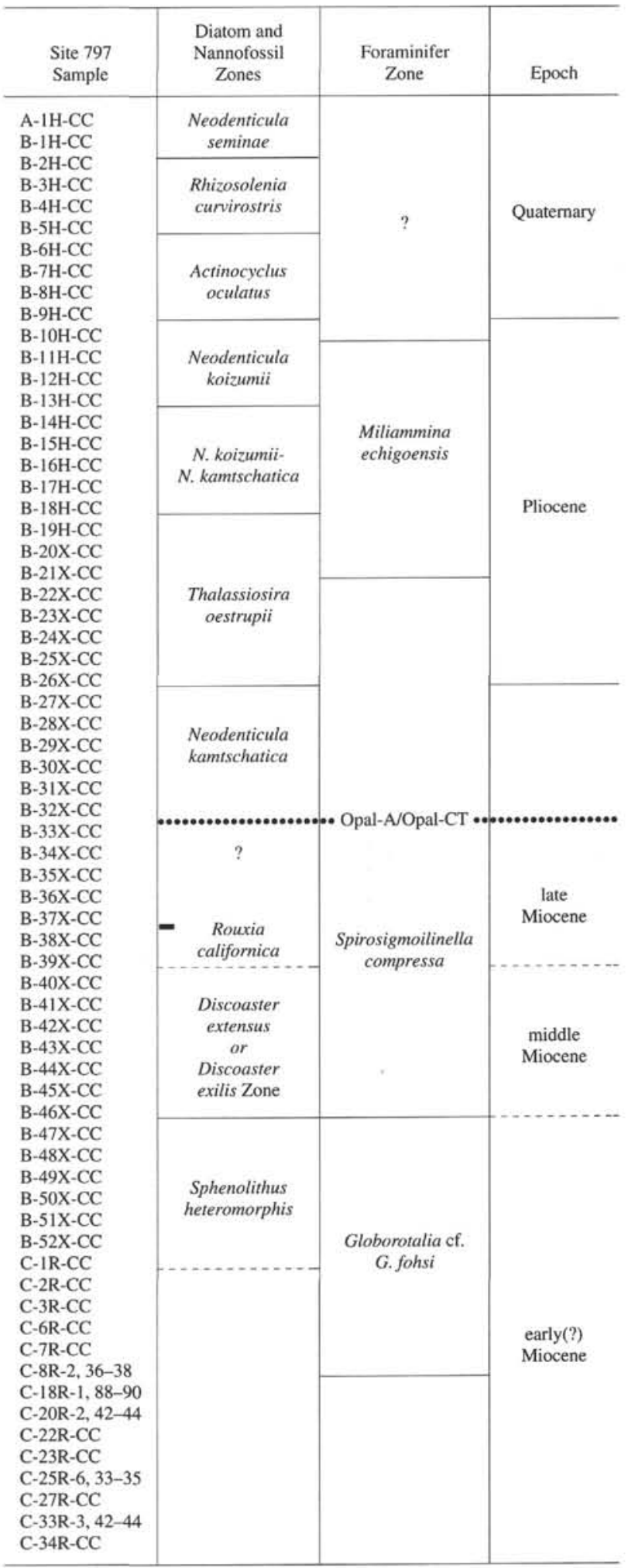


Site 797

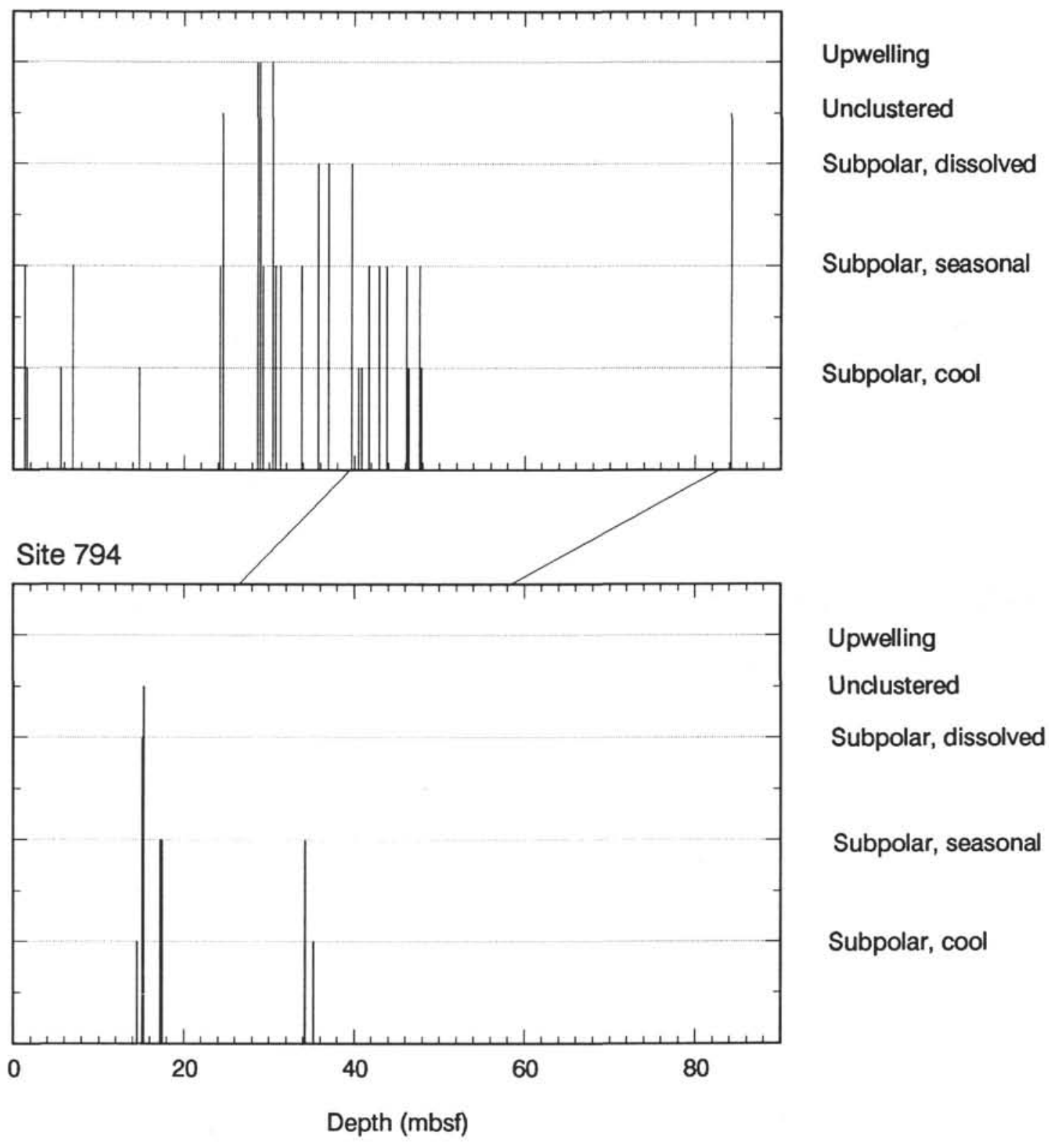

Figure 4. Succession of clusters in Holes 794A and 797B plotted against depth in hole. The subpolar cool assemblage is equivalent to Cluster 1; the subpolar seasonal assemblage is equivalent to Cluster 2; the subpolar, dissolved assemblage is equivalent to Cluster 3 ; the unclustered samples are those distinctly different from all other samples; and the upwelling(?) assemblage is equivalent to Cluster 4. The two lines connecting the graphs denote the Brunhes/Matuyama Boundary and the top of the Olduvai. 